\title{
An experimental study of entrainment and transport in the turbulent near wake of a circular cylinder
}

\author{
By BRIAN CANTWELL \\ Stanford University, Stanford, California 94305 \\ AND DONALD COLES \\ California Institute of Technology, Pasadena, California 91125
}

(Received 16 February 1983 and in revised form 6 July 1983)

This paper describes an experimental investigation of transport processes in the near wake of a circular cylinder at a Reynolds number of 140000 . The flow in the first eight diameters of the wake was measured using $X$-array hot-wire probes mounted on a pair of whirling arms. This flying-hot-wire technique increases the relative velocity component along the probe axis and thus decreases the relative flow angle to usable values in regions where fluctuations in flow velocity and direction are large. One valuable fringe benefit of the technique is that rotation of the arms in a uniform flow applies a wide range of relative flow angles to the $X$-arrays, making them inherently self-calibrating in pitch. An analog circuit was used to generate an intermittency signal, and a fast surface-pressure sensor was used to generate a phase signal synchronized with the vortex-shedding process. The phase signal allowed sorting of the velocity data into 16 populations, each having essentially constant phase. An ensemble average for each population yielded a sequence of pictures of the instantaneous mean flow field, with the vortices frozen as they would be in a photograph. In addition to globally averaged data for velocity and stress, the measurements yield non-steady mean data (in the sense of an average at constant phase) for velocity, intermittency, vorticity, stress and turbulent-energy production as a function of phase for the first eight diameters of the near wake. The stresses were resolved into a contribution from the periodic motion and a contribution from the random motion at constant phase. The two contributions are found to have comparable amplitudes but quite different geometries, and the time average of their sum (the conventional global Reynolds stress) therefore has a quite-complex structure. The non-steady mean-vorticity field is obtained with good resolution as the curl of the non-steady mean-velocity field. Less than half of the shed circulation appears in the vortices, and there is a slow decay of this circulation for each shed vortex as it moves downstream. In the discussion, considerable emphasis is put on the topology of the non-steady mean flow, which emerges as a pattern of centres and saddles in a frame of reference moving with the eddies. The kinematics of the vortex-formation process are described in terms of the formation and evolution of saddle points between vortices in the first few diameters of the near wake. One important conclusion is that a substantial part of the turbulence production is concentrated near the saddles and that the mechanism of turbulence production is probably vortex stretching at 
intermediate scales. Entrainment is also found to be closely associated with saddles and to be concentrated near the upstream-facing interface of each vortex.

\section{Introduction}

\section{Motivation}

It has become increasingly evident to many workers in turbulence research that the operation of Reynolds averaging in the equations of motion hides from view many important features of turbulent flow. Various conditional sampling and averaging techniques have recently been developed to study these features, with the hope eventually of understanding better the mechanisms of turbulence. The most important result so far is a new perception of the large eddies which account for much of the transport of momentum, heat, and mass. Until recently, a large eddy was often visualized as an accidental, energetic, large-scale motion energized primarily by the straining action of the mean flow. In the new view, a large eddy is visualized as an organized, coherent concentration of large-scale vorticity energized primarily through entrainment while retaining its geometry over many characteristic lengths of the motion. The cumulative signature of such coherent structures $i s$ the mean flow.

Although the concept of the coherent large eddy represents an advance in our physical understanding of turbulence, many issues remain unresolved. For example, it is generally accepted that the motions at the smallest scale of a flow tend to become independent of the large eddies as the Reynolds number increases. Although many measurements in different flows support this view, virtually all the evidence is based on measurements of spectra. While this evidence substantially confirms the $k^{-\frac{5}{3}}$ behaviour predicted by Kolmogorov (1941), the fact is that very little is known about the coupling between the organized motion and the smaller scales (the energy cascade), or about the mechanisms by which energy delivery and dissipation are actually accomplished.

The flow in the near wake of a circular cylinder at high subcritical Reynolds number is a flow in which coherent large eddies can be studied in their natural state. The eddies in question are turbulent line vortices which are produced and shed in an essentially regular manner. Except for some dispersion, the vortices are not subject to interactions that might obscure their identity, at least in the first few diameters downstream of the cylinder. The relative ease with which regular and irregular motions can be separated thus makes the near wake an attractive case for studying turbulent transport. In short, it is possible to identify the large eddy for this flow to observe its formation, and to trace its history as it is carried downstream. In a frame of reference fixed to the cylinder, the non-steady mean flow field takes on the appearance of a wave having a frequency equal to the shedding frequency and a phase velocity or celerity which increases continuously with increasing distance downstream. In a frame of reference moving downstream at some representative phase velocity, the instantaneous mean streamlines form a pattern of centres and saddles which delineate the individual large eddies as almost stationary vorticity concentrations of alternating sign.

The purpose of the experiment described in this paper was to observe the flow in the moving frame of reference just described. The method, described in detail in $\$ \S 2$ and 3 , was to use $X$-array hot-wire sensors on the ends of a pair of whirling arms to sample the flow at closely spaced points along an arc of relatively large diameter. 
Simultaneously, the phase of the vortex-shedding process was recorded by sampling the surface pressure at a suitable point on the cylinder model. For a large number of probe revolutions along each of many different probe ares, the data associated with each point in the flow field were sorted into 16 groups, each corresponding to a small interval for the phase of the surface pressure signal. After ensemble averaging, the result was a 16-frame animation of the non-steady mean flow in terms of intermittency, instantaneous mean velocity, instantaneous mean vorticity, and instantaneous Reynolds stresses.

\section{Analytical background}

The flow in the near wake can be viewed formally as a combination of a global mean component $\bar{s}$, a periodic mean component $\tilde{s}$, and a random component $s^{\prime}$, where $s$ is any variable. By definition, the total variable $s$ is the sum

$$
s=\bar{s}+\tilde{s}+s^{\prime} .
$$

This notation, and part of the development that follows, are borrowed from Reynolds \& Hussain (1972). $\dagger$ There are two different averaging processes which can be defined operationally in terms of manipulations actually carried out for the present digital data. At each of many points in the flow field, there is available a population of $N(=16384)$ samples $s_{n}$ from a given probe. The global mean $\bar{s}$ is defined by counting:

$$
N \bar{s}=\sum_{n=1}^{N} s_{n}
$$

This population of $N$ samples is then divided into 16 nominally equal subpopulations of $N_{i}(=1024)$ samples, each subpopulation being associated with a particular phase interval for the shedding process. The mean at constant phase $\langle s\rangle$ is again defined by counting within each subpopulation:

$$
N_{i}\langle s\rangle=\sum_{\nu=1}^{N_{i}} s_{\nu} \quad(i=1,2, \ldots, 16) .
$$

The periodic component $\tilde{s}$ is then defined as the difference

$$
\tilde{s}=\langle s\rangle-\bar{s} .
$$

It follows from these definitions, as pointed out by Reynolds \& Hussain, that $\overline{\widetilde{s}}=0$ (the periodic motion has zero mean when averaged over one cycle); that $\left\langle s^{\prime}\right\rangle=0$ (the random fluctuations have zero mean at constant phase); and that $\overline{\widetilde{s} s^{\prime}}=0$ (the periodic and random motions are uncorrelated). To implement these ideas in the Navier-Stokes equations, first put $s=\langle s\rangle+s^{\prime}$; then take \langle\rangle (the average at constant phase); then put $\langle s\rangle=\bar{s}+\tilde{s}$; then take the average over one cycle. If $s$ is velocity, each of the quantities $s,\langle s\rangle, \bar{s}, \tilde{s}, s^{\prime}$ separately satisfies the ordinary continuity equation. The momentum equation for the mean flow at constant phase has the conventional form, except for the presence of a non-steady term,

$$
\rho \frac{\partial\left\langle u_{i}\right\rangle}{\partial t}+\rho\left\langle u_{j}\right\rangle \frac{\partial\left\langle u_{i}\right\rangle}{\partial x_{j}}=-\frac{\partial\langle p\rangle}{\partial x_{i}}+\mu \frac{\partial^{2}\left\langle u_{i}\right\rangle}{\partial x_{k}^{2}}-\rho \frac{\partial\left\langle u_{i}^{\prime} u_{j}^{\prime}\right\rangle}{\partial x_{j}}
$$

$\uparrow$ Reynolds \& Hussain refer to $\bar{s}$ as time average (meaning average over time) and to $\langle s\rangle$ as phase average (meaning not average over phase, but average at constant phase). We prefer to call these quantities the global mean and the mean at constant phase, respectively. 
The momentum equation for the global mean flow (equation (2.5) of Reynolds \& Hussain) has the form

$$
\rho \bar{u}_{j} \frac{\partial \bar{u}_{i}}{\partial x_{j}}=-\frac{\partial \bar{p}}{\partial x_{i}}+\mu \frac{\partial^{2} \bar{u}_{i}}{\partial x_{k}^{2}}-\rho \frac{\left.\overline{\partial\left(\left\langle u_{i}^{\prime} u_{j}^{\prime}\right\rangle\right.}+\overline{\tilde{u}_{i} \tilde{u}_{j}}\right)}{\partial x_{j}} .
$$

The contributions of the random and periodic motions to the Reynolds stresses associated with the global mean flow are thus displayed explicitly.

Reynolds \& Hussain were concerned with the case of a small periodic disturbance $\tilde{s}$ to an established mean flow $\bar{s}$. It was therefore appropriate for them to take their global mean flow to be well defined, i.e. real in the Reynolds-averaged sense, in the absence of the disturbance. In the present work on coherent structure, it is more appropriate to write, in lieu of (1) and (4),

$$
s=\langle s\rangle+s^{\prime},
$$

and to proceed only as far as (5). It is now the periodic mean flow that is considered to be real in the Reynolds-averaged sense. The governing dynamical equation (5) includes only the conventional Reynolds stresses $\left\langle u_{i}^{\prime} u_{j}^{\prime}\right\rangle$ which are generated by spatially local random fluctuations at constant phase. These stresses directly control the dynamies of the coherent structures and thus operate at a lower level than the stresses in (6). Turbulence is interpreted as a local phenomenon, attached to and moving with the individual vortices and their connecting saddles. Phase information is retained at the largest scale of the flow.

A primary objective of the present research was measurement of the various quantities that play the role of Reynolds stresses in (5) and (6). The most important of these from the point of view of coherent structure is $\left\langle u_{i}^{\prime} u_{j}^{\prime}\right\rangle$. Of equal importance from the point of view of conventional turbulence modelling is $\tilde{u}_{i} \tilde{u}_{j}$, which is needed along with $\left\langle u_{i}^{\prime} u_{j}^{\prime}\right\rangle$ for any inquiry into the relative contributions of the periodic and random motions to Reynolds-stress terms in the global mean-momentum equation (6). Examples of these quantities are displayed and discussed in $\$ 4$ below.

\section{Experimental background}

Various measurements of drag and shedding frequency for smooth circular cylinders in the range $10^{3}<R e<10^{6}$ are collected in figure 1 . Some notes on the experiments appear in table 1. It goes almost without saying that more reliance should be placed on data for cylinders of large aspect ratio (10 or more) in situations of low blockage $(10 \%$ or less), low freestream turbulence level ( $1 \%$ or less), and low Mach number ( 0.25 or less). The stars represent values observed during the present experiments, as described in $\$ \S 3$ and 4 below. Open circles are data from experiments at one Reynolds number.

Apparently there is still some uncertainty about the drag and shedding frequency for a circular cylinder, in spite of the large number of independent observations. In the case of the drag coefficient $C_{\mathrm{d}}$, the present values are reasonably close to the values obtained by Bearman, Bursnall \& Loftin, Humphries, Kamiya et al., Miller and Polhamus (see table 1), although the agreement does not always extend to the details of the mean pressure distribution around the cylinder. In the case of the Strouhal number $S t$, the present value of 0.179 at $R e=140000$ is slightly lower than the consensus of other measurements. If any conclusion can be drawn from the experience represented by figure 1 , it is that end conditions for the cylinder model are critical. The closest approach to two-dimensional mean flow is apparently obtained by making 


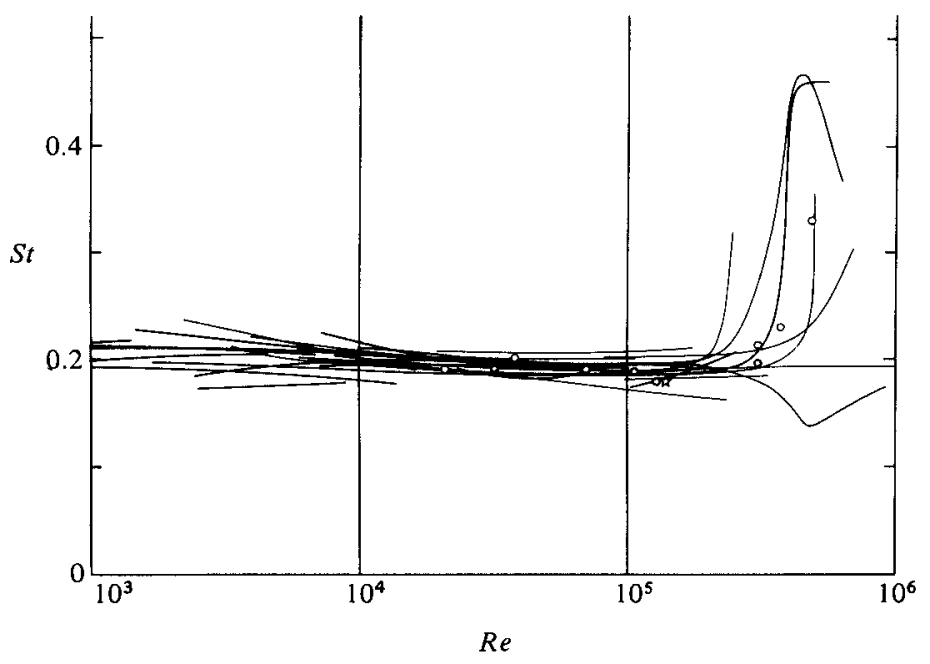

(a)

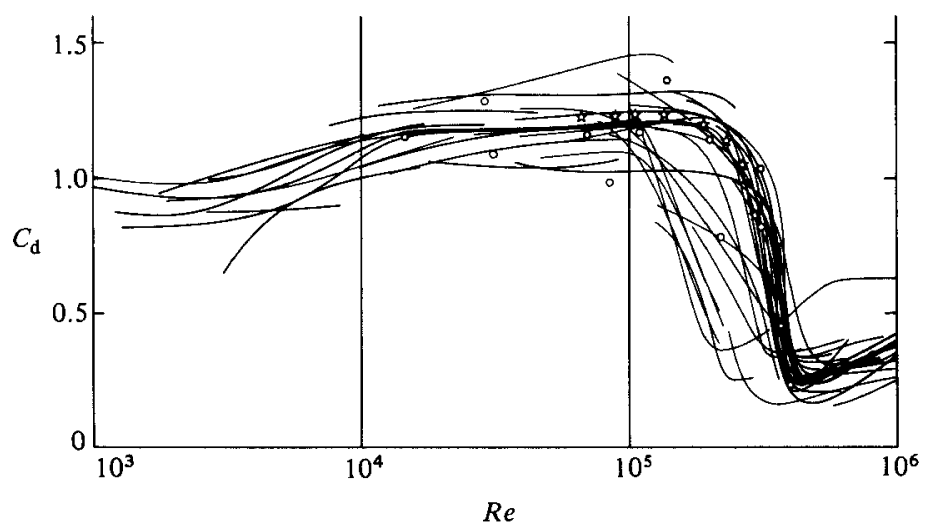

(b)

Figure 1. Collected experimental data for $(a)$ Strouhal number and $(b)$ drag coefficient for low-speed flow past a smooth circular cylinder. Data sources are listed in table 1. Stars show results from the present research.

the boundary layers on the endwalls as thin as possible, usually by the use of endplates of moderate size. Such a strategy can be effective even for relatively short cylinders.

Several experimenters have recently used digital methods to preserve phase information in studies of flow in the near wake of two-dimensional bluff bodies. Davies (1976) worked with fixed hot wires in the wake of a $D$-section body with its flat face upstream. The Reynolds number is not stated, but it appears to be about 25000 . Phase was measured from successive maxima in the nearly periodic signal from a hot wire placed outside the wake. The objective of this experiment was to compare wake flows for a fixed body and an oscillating body, rather than to map the wake flow in detail. Measurements were made only at one station, which was placed well downstream $(x / D=8)$ to minimize the problem of large relative flow angles for the hot wires. The observed time dependence at this single station is converted to spatial dependence to obtain an approximation to the mean-flow pattern which would be 


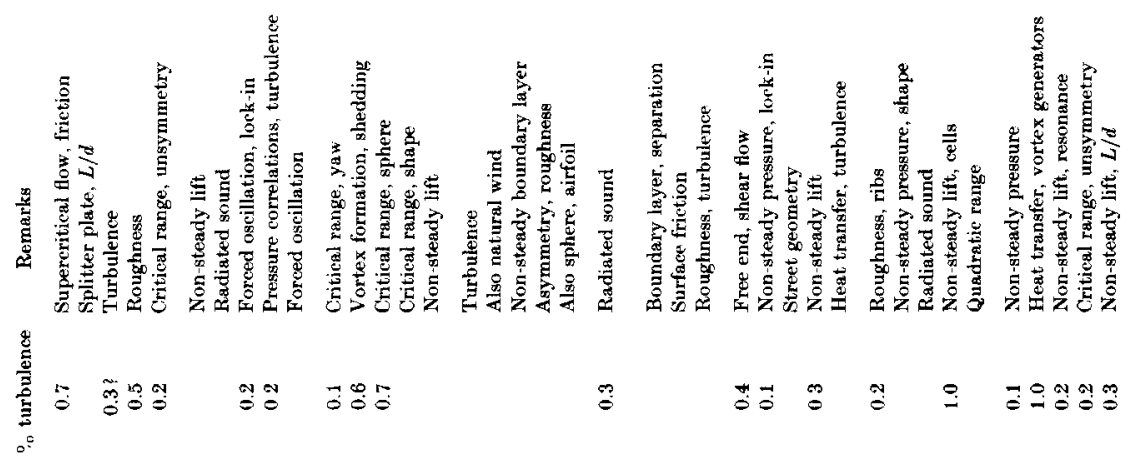

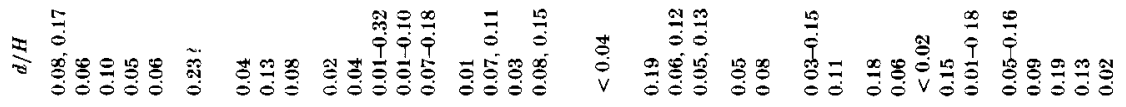

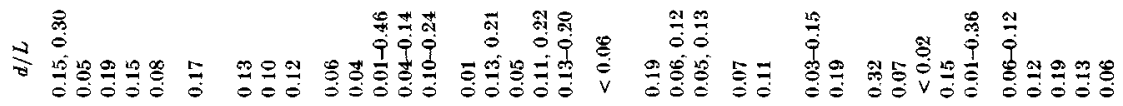

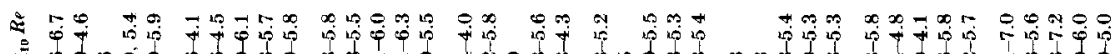

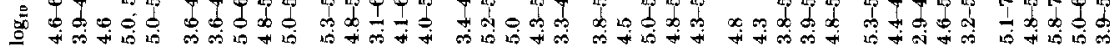

क $\exists \times x$

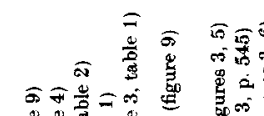

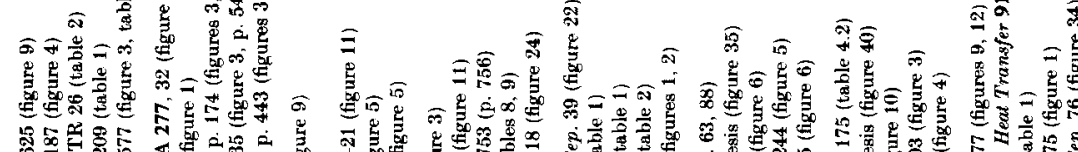

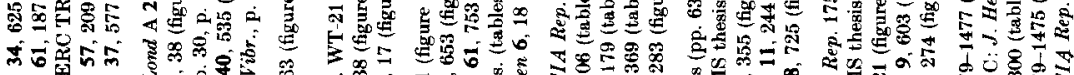

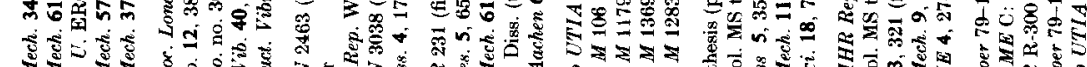

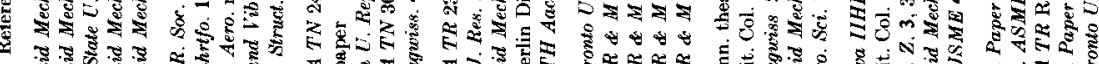

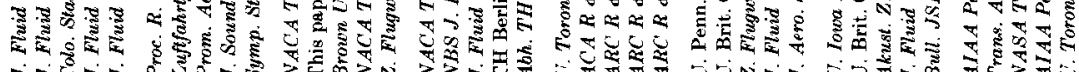

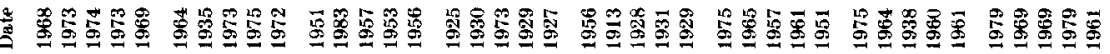

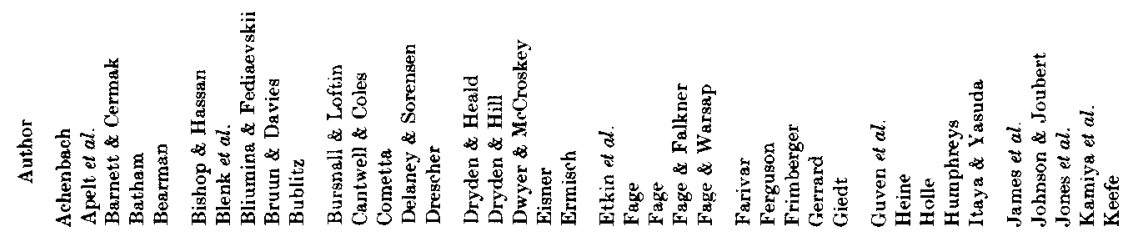




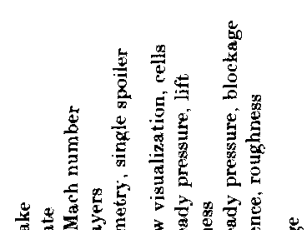

HDW

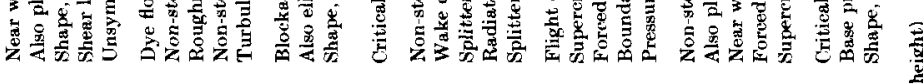

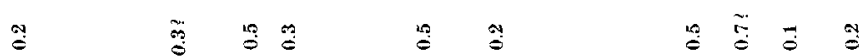

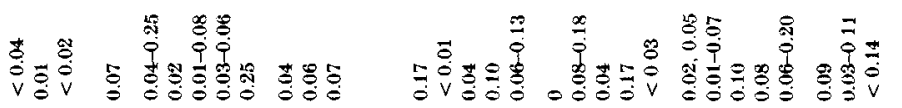

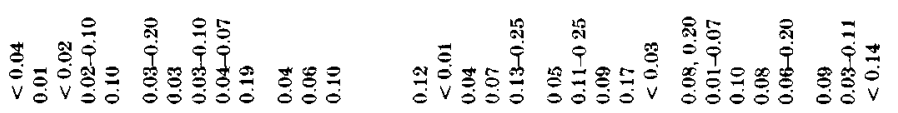

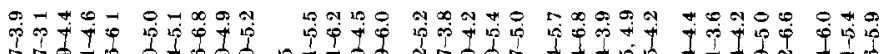

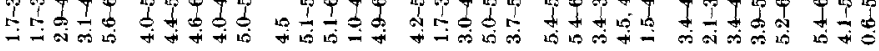

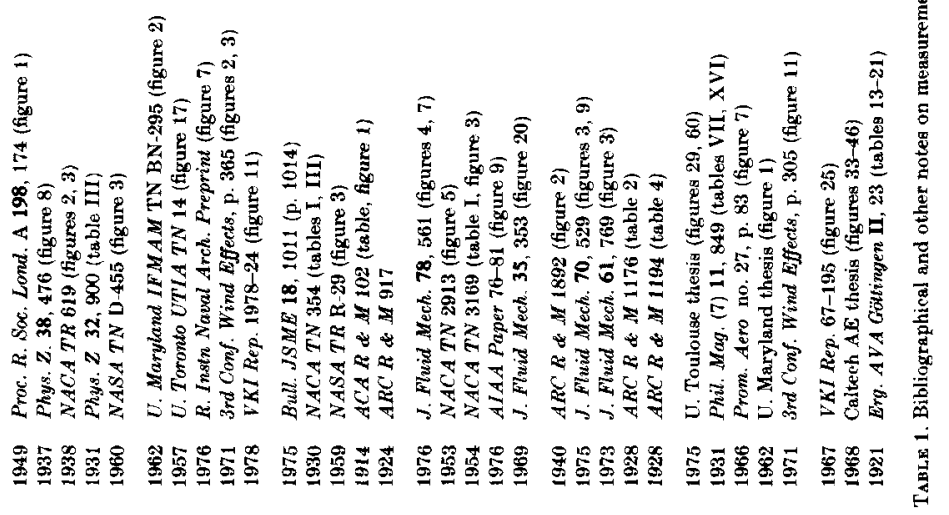

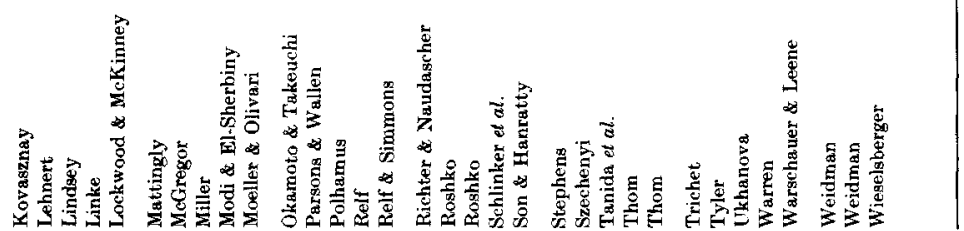


seen by an observer moving downstream at the proper phase velocity. The latter was inferred from cross-correlation of signals from two probes separated in the $x$-direction.

More recently, Owen \& Johnson (1980) used laser anemometry to measure vortex flow fields in the near wake of a cylinder at a high subsonic Mach number $\left(M_{\infty}=0.6\right)$. They used a surface hot film as a phase reference. In agreement with the present measurements, they found that the area of mean-flow reversal is relatively small, with the wake closure point occurring slightly more than one diameter downstream of the cylinder centre. However, they found significantly larger values of the normalized wake defect velocity at comparable values of $x / D$ (for example $\Delta u / u_{\infty}=0.5$ at $x / d=4$ compared with $\Delta u / u_{\infty}=0.25$ in figure $11(a)$ for the present measurements). A possible reason for the difference is the known tendency of the vortex-formation region to increase in length as the Mach number increases in the high subsonic range (Dyment, Gryson \& Ducruet 1980). The presence of shock waves on the cylinder and in the near wake above a critical Mach number of about 0.4 has been thoroughly documented by Stack (1941), Ryan (1951), Gowen \& Perkins (1953), Thomann (1959), Gorecki (1960), Naumann, Morsbach \& Kramer (1966), and Dyment et al. (1980). Although shock waves were undoubtedly present in the experiment by Owen \& Johnson, they are not resolved by the measurements at constant phase. A related effect of Mach number is a $20 \%$ increase in $C_{d}$ at $M_{\infty}=0.6$ and $R e=1.3 \times 10^{5}$, with a comparable decrease in base pressure (Gowen \& Perkins 1953). The drag coefficient and base pressure were not measured by Owen \& Johnson, and other important aspects of the experiment, such as effects of tunnel blockage and effects of statistical sampling bias on the LDV data, are not discussed.

Wlezien \& Way (1979) have used a novel thermal-tracer method to study the vortex-formation process in the base region of a circular cylinder at Reynolds numbers between 5800 and 10400 . The results published so far deal mainly with technique, and this technique has not yet been fully exploited.

Finally, Perry \& Watmuff (1981) have carried out conditionally averaged flyinghot-wire measurements in the three-dimensional wake of an ellipsoid. They concentrated particularly on the effect of 'phase jitter' on the averaging process. The main effect is to reduce the measured contribution to the total stress of the periodic part of the motion. They also find that in the three-dimensional wake only about $15 \%$ of the stress is due to the periodic motion as compared to about $50 \%$ for the two-dimensional case. Effects of phase jitter are important in the present investigation and will be discussed in $\S \S 3$ and 4 .

The present experiment goes beyond the other experiments just cited by including intermittency data and by taking full advantage of the fact that information is available simultaneously for two components of the velocity. The resolution of the measurements is high enough, and the scatter is low enough, to allow data to be differentiated with some confidence. Access to quantitative data for mean vorticity and mean strain rate as functions of position and time leads in $\$ \$$ and 5 of this paper to several useful conclusions about the kinematics and dynamics of the flow.

\section{Model and instrumentation}

\section{Cylinder model}

The cylinder model used in the experiment was a $2.97 \mathrm{~m}$ length of stainless-steel tubing with an outside diameter of $10.14 \mathrm{~cm}$. The tubing was centreless ground and lapped to an outside surface finish of about $0.3-1.0 \mu \mathrm{m}(10-40 \mu \mathrm{in}$.). Circular endplates 


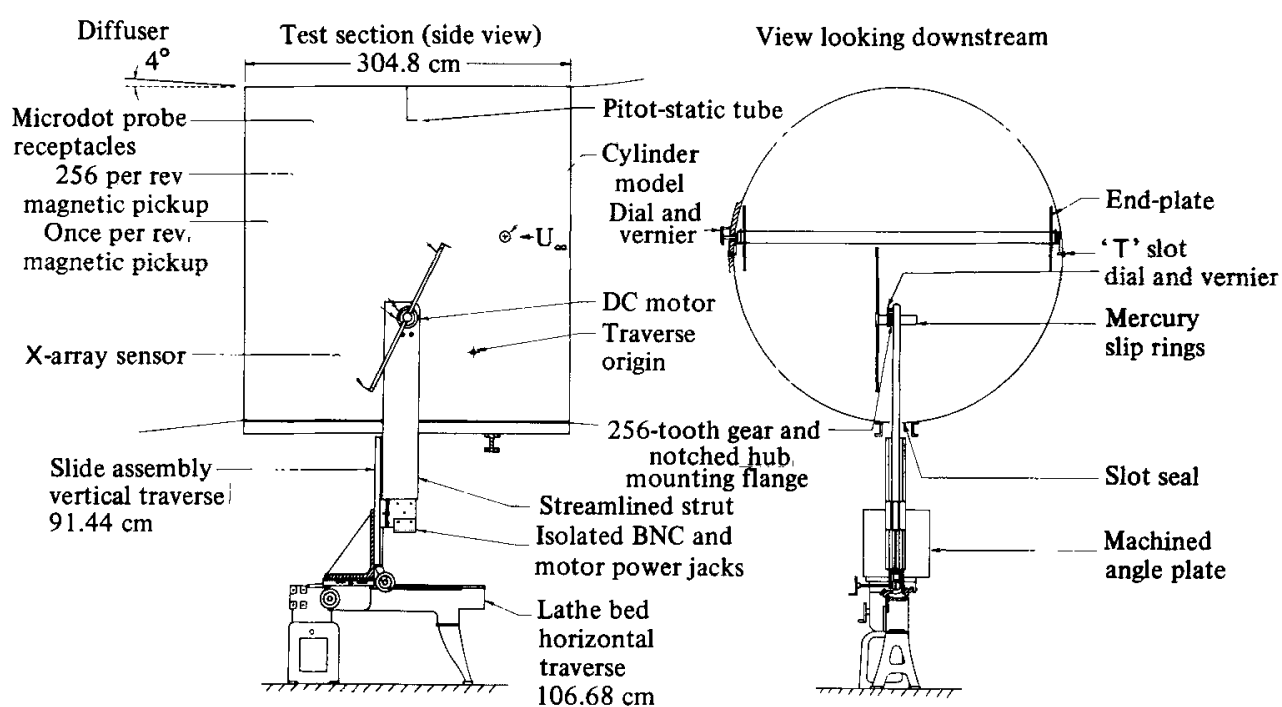

FIGURE 2. Configuration of the flying-hot-wire apparatus and cylinder model in the GALCIT $10 \mathrm{ft}$ wind tunnel.

$61 \mathrm{~cm}$ in diameter were used to isolate the flow over the cylinder from the boundary layers on the tunnel walls.

The inside of the model was honed and fitted with several sealed pistons which connected pressure holes in the wall of the cylinder to pressure instrumentation outside the tunnel. 'Two of these pressure holes, located $9.84 \mathrm{~cm}$ to either side of centre, were closely coupled to internal pressure transducers (Stow Laboratories Pitran model PT-L2; active volume $0.07 \mathrm{~cm}^{3}$ ). One Pitran transducer was used primarily to monitor the phase of the vortex-shedding cycle. The other was used briefly to verify that shedding was acceptably two-dimensional over a lateral distance of about two cylinder diameters.

\section{Flying hot wire}

A basic problem with hot-wire anemometry in regions of high turbulence level is that the instantaneous flow may approach the probe from an awkward direction. A conventional $X$-wire array, having wires mounted at $\pm 45^{\circ}$ to the probe axis, will not have a unique response if the relative flow angle is allowed to fall outside this range of $\pm 45^{\circ}$. See Tutu \& Chevray (1975) for an appreciation of this problem. The strategy of the flying-hot-wire technique is to increase the relative velocity component along the probe axis, and thus to decrease the relative flow angle, by mounting the hot-wire probes on the ends of a pair of whirling arms. One consequence is that rotation of the arms in a uniform flow automatically applies a wide range of relative flow angles to the $X$-arrays, making them inherently self-calibrating in pitch. Another consequence is that the hot-wire signals are statistically neither stationary nor homogeneous, so that the technique is necessarily digital.

Figure 2 is an assembly drawing of the flying-hot-wire apparatus in the test section of the 10 foot wind tunnel at GALCIT (Graduate Aeronautical Laboratories, California Institute of Technology). The diameter of the rotor was $151 \mathrm{~cm}$. The rotor was mounted directly on the shaft of a printed-circuit DC motor (Printed Motors model U16M4). The motor case was mounted in one end of a vertical strut which could be traversed in the streamwise and vertical directions. 
The need to maintain closely constant rotor speed in spite of large variations in torque was met by development of a phase-lock servo drive. The command input to the phase detector originated in a preset counter (in the data-acquisition system) as a $200 \mathrm{kHz}$ crystal-controlled pulse train. A series of decade counter stages divided the $200 \mathrm{kHz}$ frequency by any desired decimal integer from 1 to 999 . The feedback input to the phase detector was obtained from a 256-tooth precision gear and magnetic pickup at the rotor hub. The square-wave output of the magnetic pickup also controlled the data-sampling process. At each positive-going transition of the pickup signal, a burst of twelve samples from twelve analog channels was recorded at $200 \mathrm{kHz}$ by the data system. A second magnetic pickup and a single-tooth gear on the rotor shaft provided an index pulse once per revolution to ensure synchronization of the sampled data with rotor position. The speed range available for the flyinghot-wire rotor in still air was from about 50 r.p.m. to about 700 r.p.m. (tip speeds from 3.7 to $53 \mathrm{~m} / \mathrm{s}$ ). This still-air range was shortened at the upper end when the tunnel was running, because the servo power amplifier (Control Systems Research model 800PRA) saturated when both torque and torque fluctuation were large.

An X-array hot-wire probe was mounted in a clamp holder at the end of each arm, with the plane of the $X$ in the plane of rotation. Hot-wire signals were carried from the rotating probes to the data-acquisition system through a set of mercury slip rings (Meridian Laboratory model MSD-12).

Four channels of constant-temperature anemometry were construeted particularly for these experiments. The circuit was based on a design by Perry \& Morrison (1971). Minor changes were made to accommodate new low-drift amplifiers (Precision Monolithics model OP-05). Commercial X-array hot-wire probes were used (Disa model 55A38). The wires were platinum-plated tungsten $5 \mu \mathrm{m}$ in diameter. The nominal overheat ratio was 1.45 . The hot-wire signals were digitized and recorded directly without being processed by linearizers or other analog signal-conditioning equipment.

All probe calibrations were carried out with the tunnel empty. The operating point of the hot-wire anemometers was set with the tunnel off and with the rotor speed chosen to give a relative velocity at the sensors (encoder frequency $200000 / 94=2128$; tip speed approximately $40 \mathrm{~m} / \mathrm{s}$ ) in the middle of the range expected during the actual experiment. An internal square-wave generator was used to optimize the damping and frequency response by adjusting offset and inductance. The bandwidth inferred from this test was about $20 \mathrm{kHz}$.

A more complete description of the flying-hot-wire apparatus, including a thorough discussion of probe calibration, can be found in Cantwell (1976) and in Coles, Cantwell \& Wadcock (1978).

\section{Intermittency meter}

The conventional definition of intermittency assumes that the motion at any point in the flow at any instant can be classified unambiguously as either turbulent or non-turbulent. In particular, it is common practice to call the motion turbulent if there is appreciable energy at high frequencies. In the present experiments, because of the low sampling frequency, the classification process was carried out by analog methods in real time. Continuous analog signals from the two wires of one $X$-array were separately differentiated, added, bandpass filtered, rectified, and fed into a comparator with an adjustable threshold level. The irregular output pulse train was fed to a retriggerable one-shot whose output remained high whenever the time interval between input pulses was less than the pulse width of the one-shot. The 
output of the intermittency circuit was a TTL-compatible digital signal which was recorded as the least significant bit of each data sample. The circuit parameters (pass band, threshold level, one-shot pulse width) were adjusted until the intermittency output consistently confirmed a subjective judgment that turbulence was intermittently present or absent in a wide variety of analog hot-wire signals typical of the actual experiment.

\section{Vortex tracker}

Some means was required to measure and record the phase of vortex shedding from the cylinder. The somewhat irregular shedding frequency (about $37 \mathrm{~Hz}$ ) was low compared with the sampling frequency $(200000 / 172=1163 \mathrm{~Hz})$, so that direct recording of the shedding signal was feasible. However, recovery of phase information during later data processing would then require detailed examination of the phase signal for many millions of shedding cycles. To avoid this very substantial computing task, the shedding phase was also computed by analog methods in real time. The main Pitran pressure signal was bandpass filtered and tracked by a phase-lock loop whose centre frequency was set to the mean vortex-shedding frequency. A linear ramp signal synchronized with the vortex shedding was generated by amplifying the square-wave output of the phase-lock loop and using the positive edge to discharge the feedback capacitor of an analog integrator having a constant-voltage input. This ramp signal was sampled and recorded along with the hot-wire signals.

\section{Pressure, temperature}

Differential pressures were measured by two electronic manometers (Barocel model $1014 \mathrm{~A} / 511$ ) having a range of $0-100 \mathrm{mmHg}$ and linearity to better than $0.5 \%$ over a wide dynamic range. Tunnel dynamic pressure was monitored using a Pitot-static tube fixed midway along the ceiling of the test section. Tunnel temperature was measured by an electronic thermometer (National model LX5606) mounted on the sidewall of the test section. All of these quantities were recorded by the data system for later use in processing of hot-wire signals.

\section{The experiment}

\section{Choice of Reynolds number}

The main objective of the present research was to study the processes of vortex formation and vortex shedding in the turbulent near wake of a circular cylinder. The complexity of the experiment meant that detailed measurements would be made only at a single Reynolds number. This Reynolds number should be large enough to ensure a fully turbulent near wake, but small enough to ensure a laminar separation. This requirement anticipates the use of the results as a test case for advanced computation codes. $\dagger$ The model design should also be such as to avoid, so far as possible, deleterious effects due to the various factors (aspect ratio, blockage, roughness, etc.) which are known to contribute to the scatter in figure 1.

Figure 3 shows some preliminary measurements of mean surface-pressure distrib-

$\dagger$ The main experimental results have been recorded on magnetic tape as part of the AFOSRStanford Data Library created for the 1980-81 AFOSR-HTTM-Stanford Conference on Complex Turbulent Flows. The quantities recorded on tape include the non-steady mean and global mean velocities and Reynolds stresses as well as global mean surface pressure. Further information about the data library can be found in Kline, Cantwell \& Lilley (1981). 


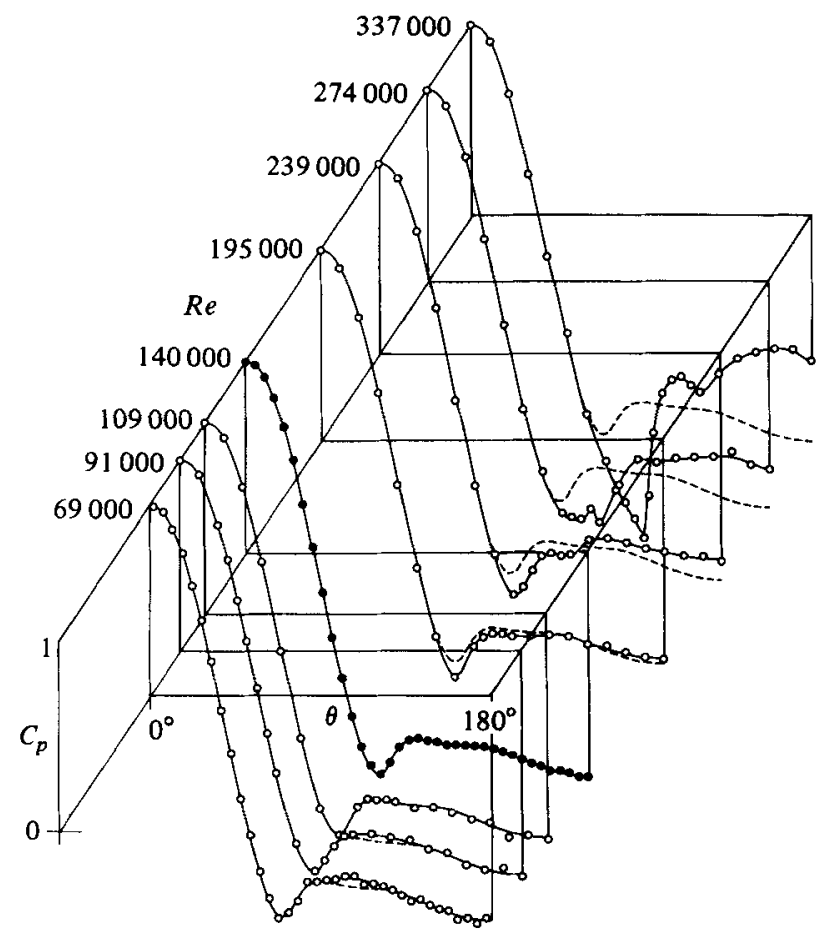

Figure 3. Model surface-pressure distribution measured at various Reynolds numbers. Flow represented by solid symbols was chosen for detailed study.

Cylinder diameter $d=10.137 \mathrm{~cm}$

Nominal arm radius $R=75.67 \mathrm{~cm}$

Dynamie pressure for wake data runs $Q_{\infty}=1.955 \mathrm{mmHg}$ nominally $5 \mathrm{lb} / \mathrm{ft}^{2}$

Freestream speed $u_{\infty}=2120 \pm 10 \mathrm{~cm} / \mathrm{s}$

Arm-tip speed for wake data runs $\omega R=2159.56 \mathrm{~cm} / \mathrm{s}$

Freestream temperature $T_{\infty}=24 \pm 2{ }^{\circ} \mathrm{C}$

Kinematic viscosity $\nu=0.1535 \mathrm{~cm}^{2} / \mathrm{s}$

Reynolds number $R e=u_{\infty} d / \nu=140000$

Freestream turbulence level; $\left(\overline{u^{\prime 2}}\right)^{\frac{1}{2}} / u_{\infty} \leqslant 0.006$

Drag coefficient $C_{\mathrm{d}}=1.237$

Base pressure coefficient $\left(180^{\circ}\right) ; C_{p_{b}}=-1.21$

Shedding period $T=26.65 \mathrm{~ms}$

Strouhal number $S t=0.179$

TABLE 2. List of experimental parameters

ution around the cylinder at various Reynolds numbers from 70000 to 340000 (these are the measurements represented by the stars in figure 1). The surface pressure was observed over one side of the model only. No correction has been made for blockage effects. The particular pressure distribution shown by solid symbols in figure 3 (and repeated as a dashed line for comparison with the other distributions) corresponds to the opera ting condition at $R e=140000$ finally chosen for the detailed measurements in the near wake. Table 2 gives a list of experimental parameters.

An important consideration in the choice of cylinder Reynolds number was the regularity of the vortex-shedding process. Tunnel speed was varied over a wide range, 


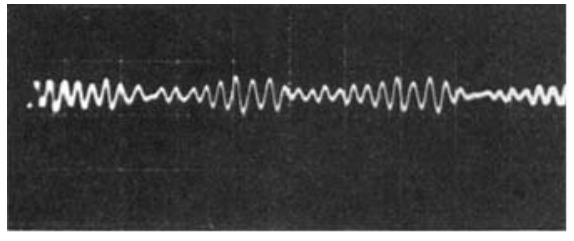

(a)

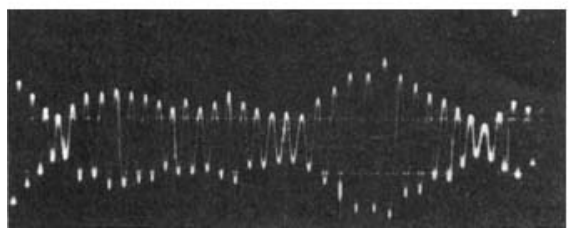

(c)

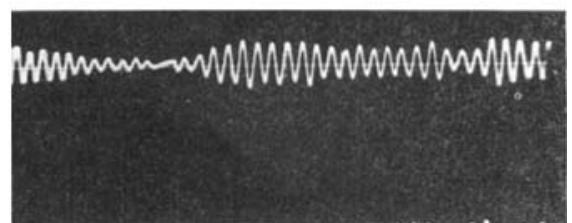

(b)

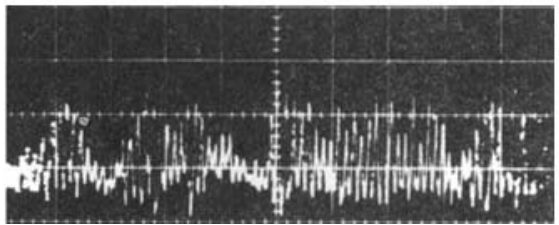

$(d)$

Figure 4. Pitran surface pressure signals at various angles away from the forward stagnation point $\left(0^{\circ}\right)$. (a) Pitran tap at $20^{\circ}$; horizontal $100 \mathrm{~ms} / \mathrm{div}$., vertical $0.02 \mathrm{~V} / \mathrm{div}$. (b) Pitran tap at $30^{\circ}$; horizontal $100 \mathrm{~ms} / \mathrm{div}$., vertical $0.02 \mathrm{~V} / \mathrm{div}$. (c) Pitran tap at $65^{\circ}$; horizontal $100 \mathrm{~ms} /$ div., vertical $0.02 \mathrm{~V} /$ div. (b) Pitran tap at $150^{\circ}$; horizontal $200 \mathrm{~ms} /$ div., vertical $0.05 \mathrm{~V} / \mathrm{div}$.

and the Pitran sensor was positioned at various angles to the flow. Typical pressure signals are shown in figure 4. Throughout the range of conditions observed, the pressure signal was characterized by the same kind of amplitude and frequency modulation which has been reported by other investigators (see e.g. Ferguson, Gerrard, Humphries and McGregor in table 1). For the main wake measurements the Pitran sensor was positioned $65^{\circ}$ away from the forward stagnation point. Lowfrequency modulation and high-frequency noise were removed with a bandpass filter before the signal was put into the vortex tracker described in $\$ 2$.

\section{Data base}

Figure 5 shows an oscilloscope record of typical hot-wire traces from the two wires of one $X$-array during one full revolution of the rotor arm. Hot-wire data were recorded only for the probe which was currently advancing into the stream; i.e. for the left half of the oscilloscope photograph, or for the upper half of the probe trajectory in figure 5 .

With two wire arrays working, the flying arm made 16384 revolutions for each position of the rotor hub (with one wire array working, it made 24576 revolutions), yielding for each probe a population of nominally 1024 samples (1536 samples) for each frame and for each of the 16 phase intervals of the shedding cycle. Of the 128 frames of data in the upper half-revolution, 50 frames (distributed partly at the beginning of the arc, partly at the end) were normally discarded as being outside the region of interest. Data were obtained with the rotor hub located at 44 different positions. The useful data base amounted to about 210000000 words of hot-wire data. Also recorded were about 70000000 words of phase information and about 60000000 words of hot-wire calibration data, pressure data, and miscellaneous information. All of these data were fully processed. 


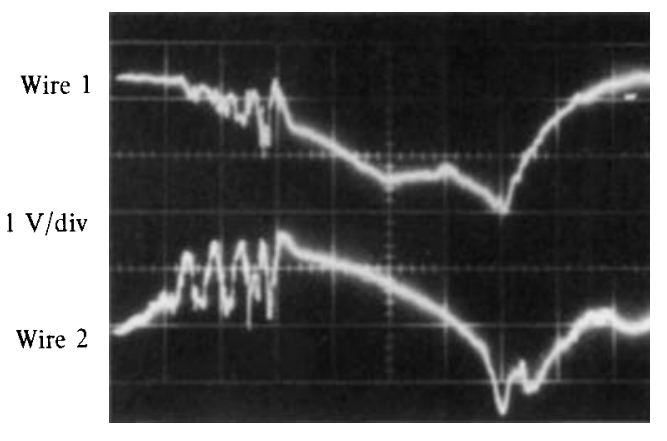

A $\quad B \quad C$

$D$

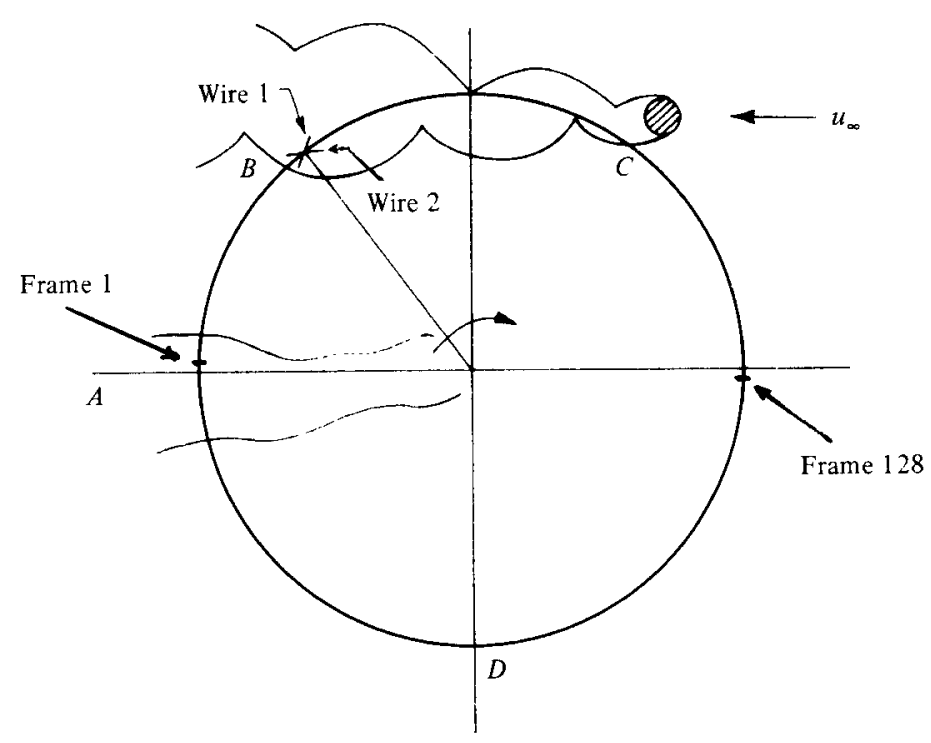

Figure 5. Typical hot-wire signals from the two wires of an $X$-array during one full revolution of the flying-hot-wire rotor. Data were recorded only for frames 1-128 of the upper half-revolution.

\section{Dispersion}

Proper assignment of phase is important because the average vortex moves (at $x / d=3.75$, say) about $0.26 d$ between phases (see $\$ 4$ and table 3 below). The method used to compute phase from ramp data is illustrated in figure 6. Each cycle of the linear ramp signal was extrapolated to zero amplitude to define an origin for the cycle. The calculation was carried to an accuracy of one octal place (i.e. $\frac{1}{8}$ of the period between frames). The interval from one ramp origin to the next was then divided into 16 subintervals, to the same accuracy, and an integer phase $1,2, \ldots, 15,16$ was assigned to each frame of data within the ramp cycle. In effect, the clock which recorded time during the experiment was reset to run at a slightly different constant rate during each vortex-shedding cycle.

The probability density for ramp intervals determined in this way is shown in figure $7(a)$. The observed dispersion in ramp interval is of one piece with the low-frequency modulation shown in figure 4 . In view of the possibility that minor 


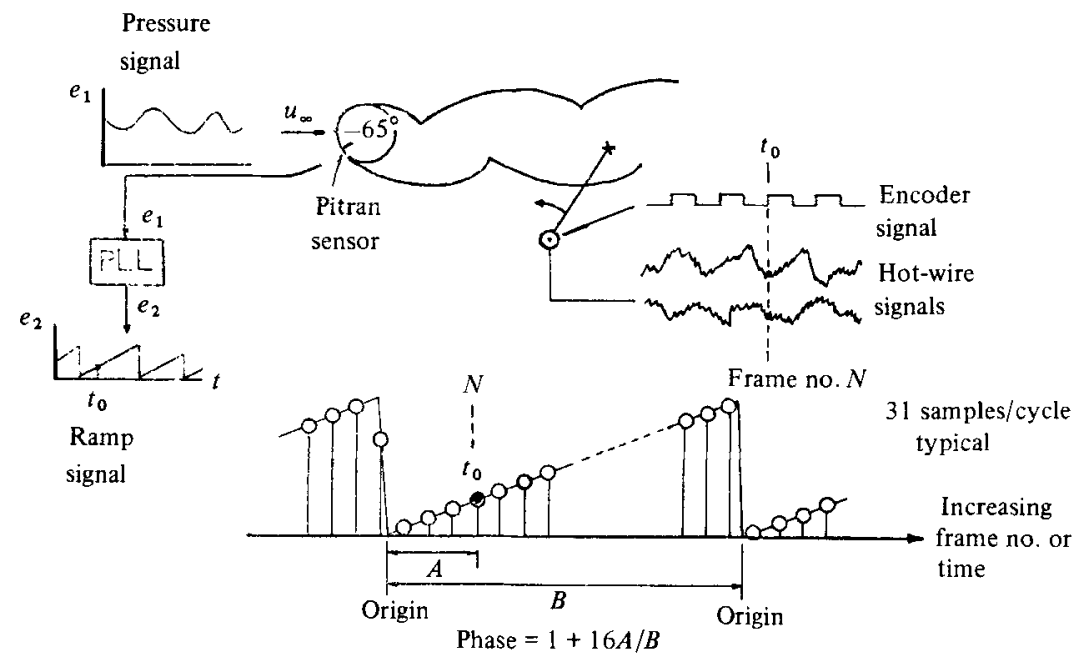

FIaURE 6. Sketch showing how ramp data were used to assign phase information to the hot-wire data.

editing of the data might lead to major improvement in the description of the flow, careful attention was paid to the problem of dispersion before the hot-wire data were finally processed. To keep this question separate from the question of probe calibration, the main variable considered was the mean intermittency $\langle\gamma\rangle$, defined as the fraction of samples in a given population which had been classified as turbulent by the circuit described in $\S 2$ (the criterion was the presence or absence of appreciable energy at scales of a few millimetres). It was found that the measured value of $\langle\gamma\rangle$ reached unity only in the region of intense turbulence near the base of the cylinder. Peak values near the vortex centres farther downstream were in the range from 0.8 to 0.9 (cf. figures 8 and 23 below). On the premise that this behaviour was caused by dispersion rather than by inhomogeneities in turbulent energy within individual vortices, various editing schemes were tried in an effort to increase the peak values.

One scheme was to discard data corresponding to the tails of the distribution in figure $7(a)$. Figure $7(b)$ shows the result for one relatively busy probe arc. Data were accepted only if several successive ramp intervals fell within a specified window centred on the mean interval. The phase was kept fixed in the sense that it was required to have a particular value for a particular frame near the cylinder. The array of binary digits at the top of figure $7(b)$ shows the raw intermittency observed during several (non-consecutive) revolutions for which this phase condition was met but no other restriction was put on the data. As the width of the window was decreased, the dispersion was decreased (i.e. the excursions in $\langle\gamma\rangle$ became more conspicuous). However, there was no substantial improvement until the population was reduced to a very small fraction of its original size. Whatever flow is being described by the remaining data, it is not the flow which is observed in nature. Similar lack of improvement was obtained with other schemes, including one in which the phase for each frame was determined from the ramp signal at an earlier time such that a vortex formed at that time would (on the average) just have reached the probe position at the moment when probe data were recorded.

Our final conclusion was that the observed dispersion is irreducible. The shedding process itself does not define the lowest frequency or the largest scale of the actual 


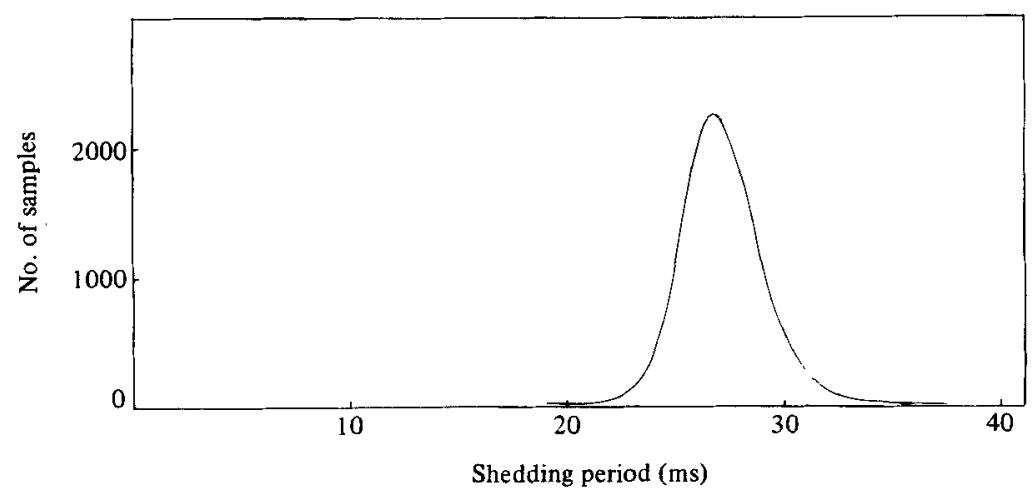

(a)

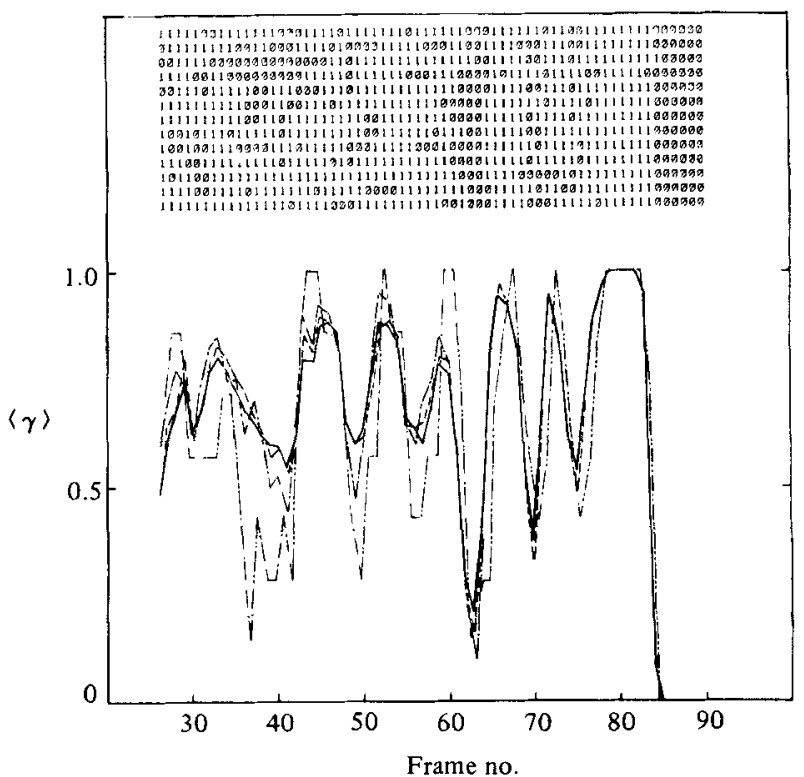

(b)

Figure 7. Effect of editing on dispersion. (a) Probability density for ramp intervals. (b) Mean intermittency distribution along one arc at phase 16. Closest approach of probe to cylinder is at frame 83. Nominal population for each frame is 1024 . Unedited raw intermittency data are also shown for several revolutions. - - All data retained; --, $78 \%$ retained; $-\cdot, 30 \%$ retained; - ..-, $5 \%$ retained.

motion, at least for a stationary cylinder. Different vortices follow different paths at different speeds, probably with considerable three-dimensionality at the scale of the variable $\left\langle u_{i}\right\rangle$ which appears in $(5)$. If variations in individual vortex trajectories in space and time are not closely correlated with pressure or velocity events occurring in the same plane very close to the cylinder, there is little point in attempting to reduce dispersion by making local adjustments to the clock of the experiment.

In the end, no data were discarded. Phase was assigned according to the local state of the Pitran pressure ramp at the time of each sample.

\section{Rotor interference}

In the present experiment, the rotor tip speed and the freestream speed were very nearly equal. Fluid in the freestream travelled more than 2 metres through the test 


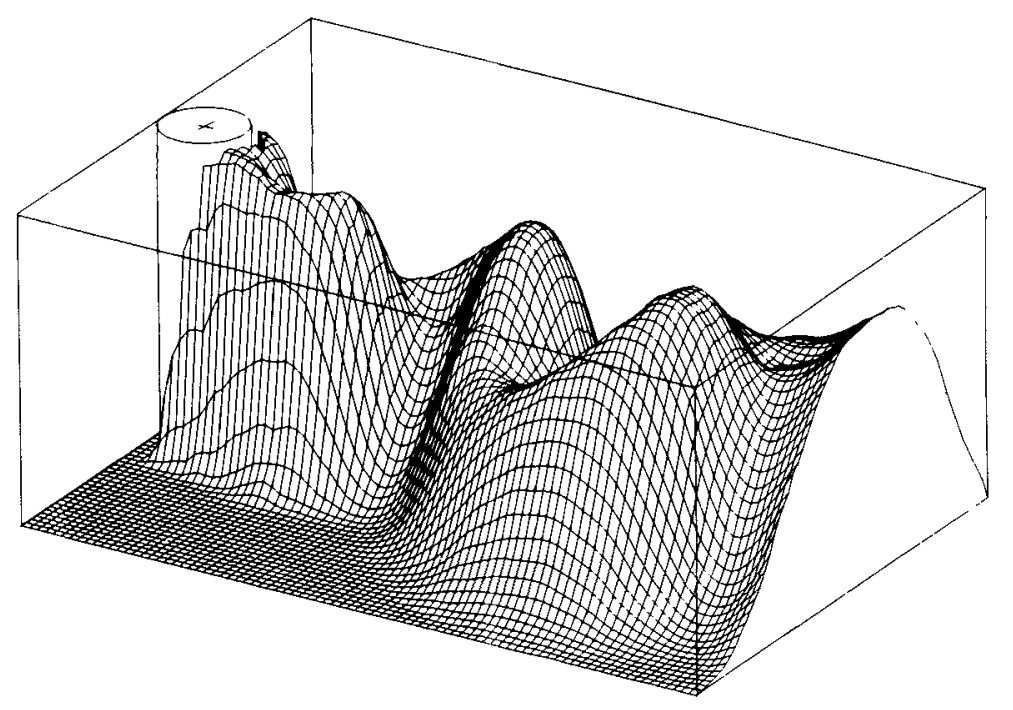

Figure 8. One frame from an animated film showing $\langle\gamma\rangle$ as a function of $x, y$ and phase.

section during each half-revolution of the rotor. It therefore seemed a reasonable presumption that effects of rotor interference might be small.

The most extensive test for interference was again made using intermittency information. Another quite different issue was explored at the same time; namely, the issue of data presentation. The results of the present experiment are capable of generating hundreds of plots depicting various correlations for periodic and for random fluctuations for the $\mathbf{1 7}$ phases of the motion (if the global mean is included). A first attack on the presentation problem produced an animated film showing the three-dimensional intermittency surface $\langle\gamma\rangle$ as a function of $x, y$ and $t$. A frame from this film is reproduced here as figure 8 . This method of data presentation was very quickly judged to be glamorous, expensive and uninformative. Simple contour plots are at least as useful, especially because they lend themselves to easy comparison of different variables by superposition of figures.

When figure 8 was being constructed, small but definite discrepancies in $\langle\gamma\rangle$ were noticed in certain regions. The intermittency surface appeared to be slightly different for different directions of probe motion. The correction for exit lag described by Coles et al. $(1978, \S 4.3)$ was applied to the data and was found to be completely effective in removing the discrepancies. Because no evidence of any systematic disturbance was found in any of the other data of the experiment, our conclusion is that rotor interference was in fact negligible. $\dagger$

\section{Data processing}

The two main operations during data reduction for this experiment were sorting of the data into populations for a given probe, are, frame, and phase, and inversion of the hot-wire data to obtain sampled velocity vectors. These operations were not completely independent. Substitution of a linear ramp signal for the oscillating surface pressure reduced the assignment of phase to a simple calculation using binary

+ In an experiment involving measurements with the same flying-hot-wire apparatus in the wake of a stalled airfoil, a different and unexpected kind of rotor interference was encountered. This problem is described and discussed by Coles \& Wadcock (1979). 


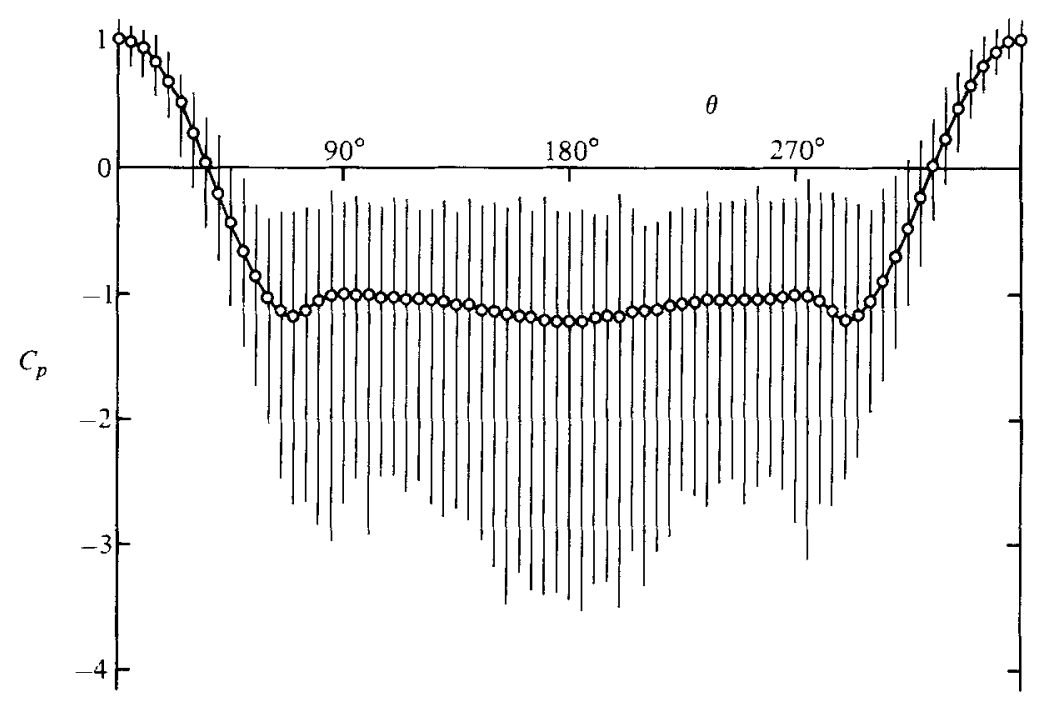

Figure 9. Measured global mean and fluctuating surface pressure at a Reynolds number of 140000. Vertical lines connect largest and smallest instantaneous pressures observed at each point during several thousand shedding cycles.

arithmetic. If the hot-wire calibration had been free of drift, the inversion operation from voltage to velocity would have been almost trivial. Each voltage pair for a given probe could be assigned to some cell in a finite discrete master array of (say $250 \times 250$ cells. Each population corresponding to a given arc, frame and phase would then generate its own two-dimensional histogram in some region of the master array. The voltage pair for each of the 62500 cells in the array would have to be inverted only once. Velocity means and moments for the various populations would then follow on weighting and summing the velocity values for the various cells. The saving in computer time, compared with the prospect of 105000000 separate inversions, would be substantial. In practice, essentially this scheme was used, except that drift in the hot-wire calibrations required most of the master array to be inverted for each of the 44 data ares, and the number of inversions actually required was closer to 2000000. This processing is described in considerable detail elsewhere (Cantwell 1976).

\section{Presentation of results}

\section{Global mean flow}

Figure 9 shows the result of a careful second measurement of the conventional mean surface-pressure distribution around the cylinder at $R e=140000$. The open symbols denote the mean pressure at each point. $\dagger$ The drag coefficient is 1.237 and the

$\dagger$ The pressure coefficients $C_{p}=2\left(p-p_{\infty}\right) / \rho u_{\infty}^{2}$ in figures 3 and 9 and throughout this paper are referred to static and dynamic pressures indicated by the ceiling-mounted Pitot-static tube. A small but consistent difference in total pressure was observed between values at this position and at the model position. For example, the measured value of $C_{p}$ at the forward stagnation point of the cylinder at $R e=140000$ was 1.019 , not 1.000 . This discrepancy was checked by interchanging the two pressure transducers and found to be real. The discrepancy is presumably due to non-uniformity in total pressure in the upstream flow; i.e. it is inherent in the low-speed operating characteristics of the 10 foot tunnel with the cylinder model installed. 


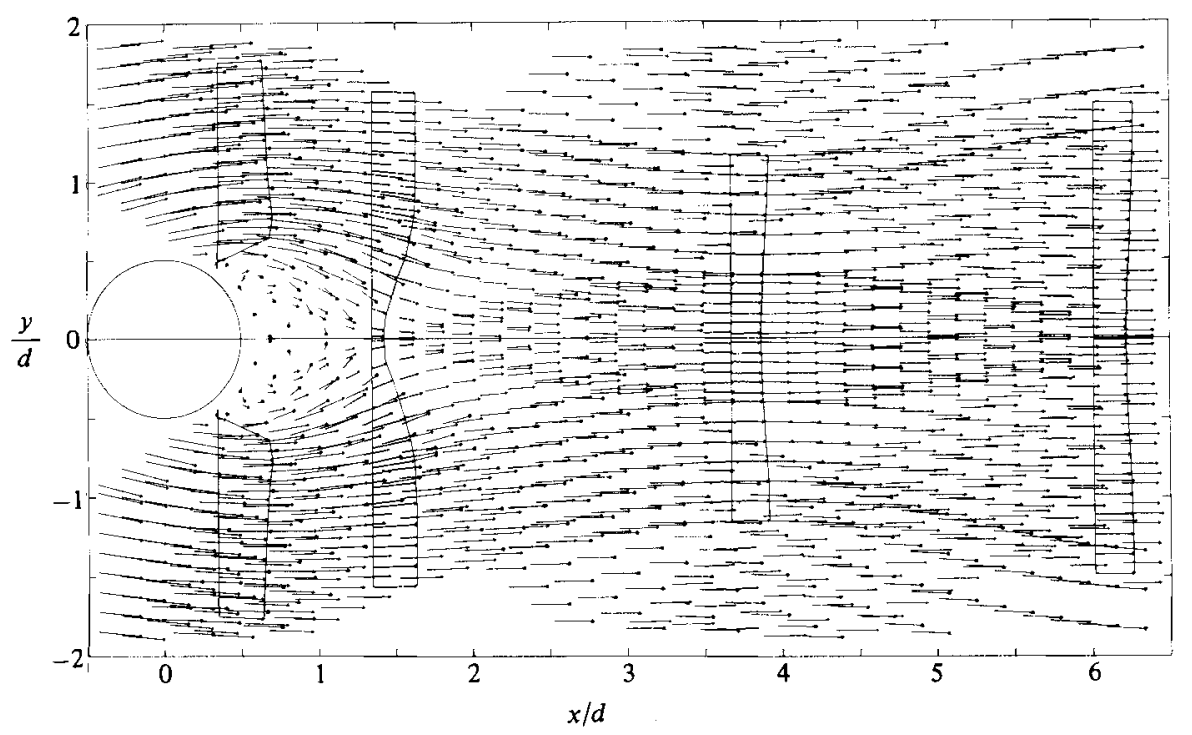

FIGURE 10. Velocity vectors for the global mean flow as measured at equally spaced points along the three families of flying-hot-wire arcs. All data have been reflected in the plane of symmetry.

base-pressure coefficient is -1.21 . The latter value varied less than \pm 0.02 over the central 20 diameters of the cylinder $\operatorname{span}$ (length/diameter $=27 / 1$ ). The mean separation position (here identified with the inflection point for the mean pressure coefficient) is about $77^{\circ}$ away from the forward stagnation point. The vertical lines in figure 9 connect the largest and smallest instantaneous pressures ever observed during several thousand shedding cycles. Note that the pressure excursions are not arbitrarily large, but appear to be bounded above and below by envelopes having the same general shape as the distribution of mean pressure. The instantaneous base-pressure coefficient can be as high as -0.2 or as low as -3.5 .

Figure 10 shows the global mean velocity in the near wake as obtained from the flying-hot-wire data. There are three families of arcs in the figure, each produced by traversing the rotor hub vertically while keeping the horizontal position fixed. Here and in later figures the spatial redundancy of the data has been fully exploited by reflecting the probe ares in the plane of symmetry.

The most striking feature of figure 10 is the small size of the mean separation bubble. The mean closure point appears to be slightly more than half a diameter downstream of the rear stagnation point. There are very few data points in and near the zone of recirculating mean flow, and some of these have had to be discarded because an appreciable fraction of the relative velocity vectors fell outside the practically useful angular range of the hot-wire array $\left( \pm 30^{\circ}\right)$ for certain phases of the shedding cycle, in spite of the strong velocity bias. It is relevant here that measurements of the mean flow are more sensitive than measurements of fluctuations to errors arising from rotor interference or from the transformation from relative to absolute velocity. Rotor interference, if present, should appear in figure 10 in the form of discrepancies in flow direction where arcs at widely different angles intersect each other. This is not the case. The occasional small discrepancies in flow direction which are observed are probably due primarily to drift and other inaccuracies in the hot-wire calibrations.

Another feature of figure 10 is the rapid relaxation of the mean-velocity defect in the plane of symmetry. The global mean velocity in this plane is shown in figure $11(a)$. 


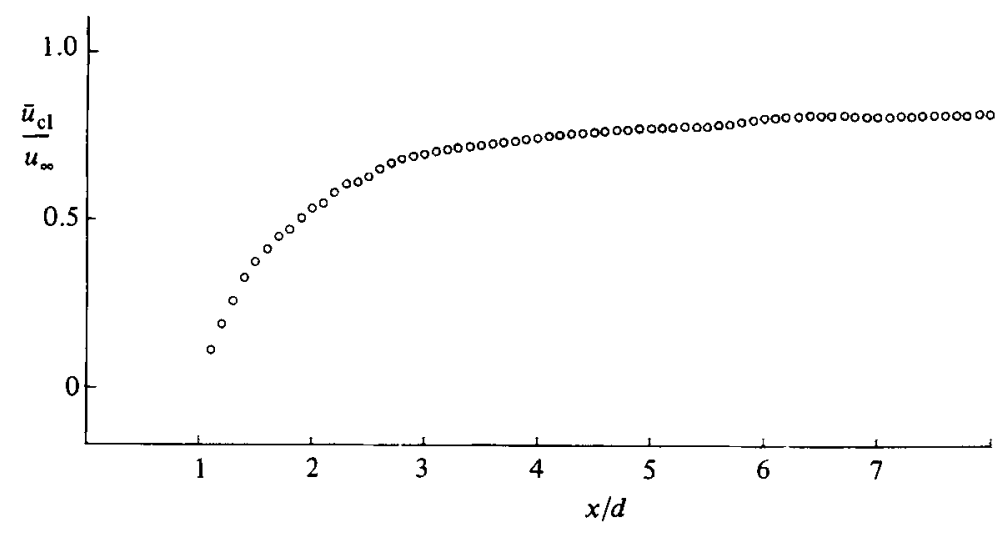

(a)

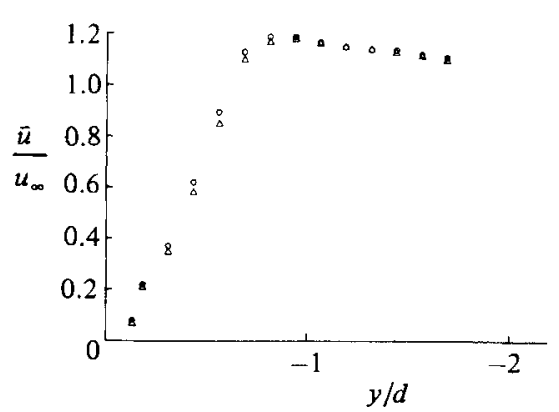

(b)

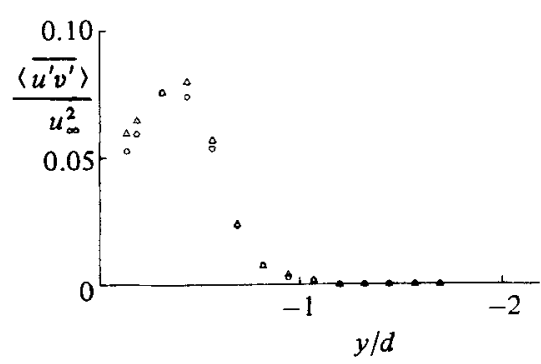

(c)

Figure 11. Typical mean-flow measurements. $(a)$ Global mean centreline velocity $\bar{u}_{\mathrm{cl}} / u_{\infty}$ as interpolated from the three overlapping sets of ares in figure 10 after averaging data from two wire sets. (b) Global mean velocity $\bar{u} / u_{\infty}$ at $x / d=1.0$ as measured by wire set $1(O)$ and wire set $2(\triangle)$. (c) Global mean shearing stress $\overline{\left\langle u^{\prime} v^{\prime}\right\rangle} / u_{\infty}^{2}$ due to random turbulence at $x / d=1.0$ as measured by wire set $1(O)$ and wire set $2(\triangle)$.

The rapid downstream acceleration of the mean flow in the face of an upstream pressure force is evidence of very powerful mixing processes in the near wake.

\section{Mean flow at constant phase}

Figures 12-19 show the velocity field at eight phases during one half of the shedding cycle. The second half of the shedding cycle simply repeats the data in these eight figures after reflection of each figure in the plane of symmetry. Only one family of ares, the one with the most uniform hot-wire calibration and the least difficulty with relative flow angle, is used to construct these figures. As in figure 10, this family is reflected in the plane of symmetry in order to produce a coherent picture from data which were taken mostly below the wake centerline. Direct and reflected data are from the same run but are $180^{\circ}$ apart in phase, so that the sign of the $v$-velocity is reversed for the reflected data. In general, the data show excellent agreement everywhere in the common region.

Figures 12(a)-19(a) represent the mean velocity field as it would appear to an observer moving downstream at a velocity of $0.755 u_{\infty}$. This particular velocity 


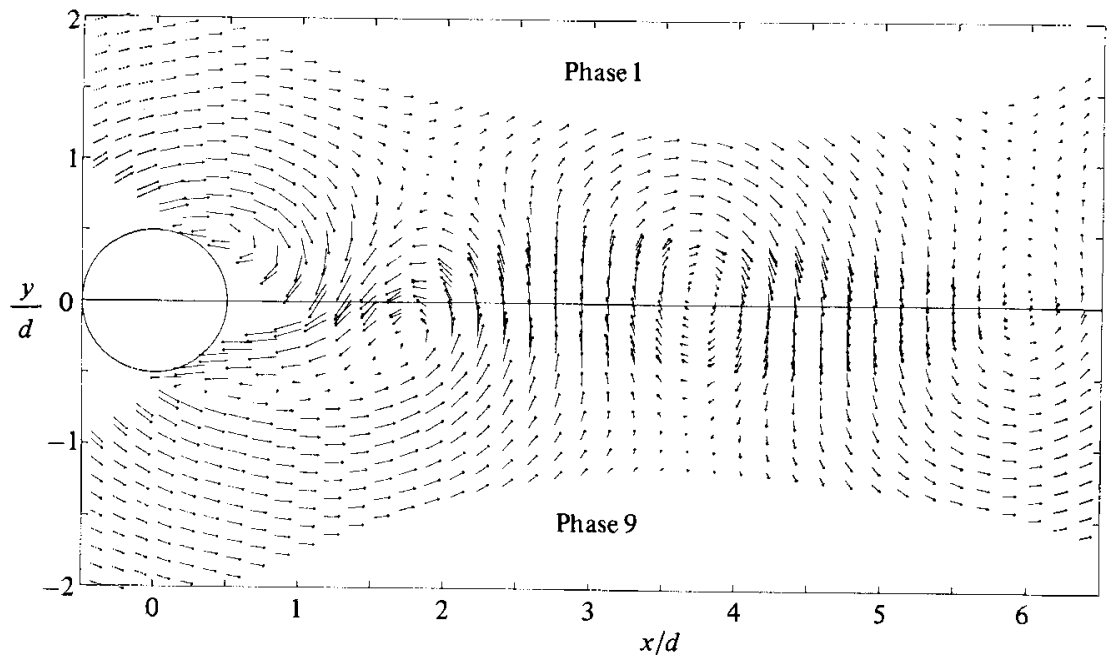

(a)

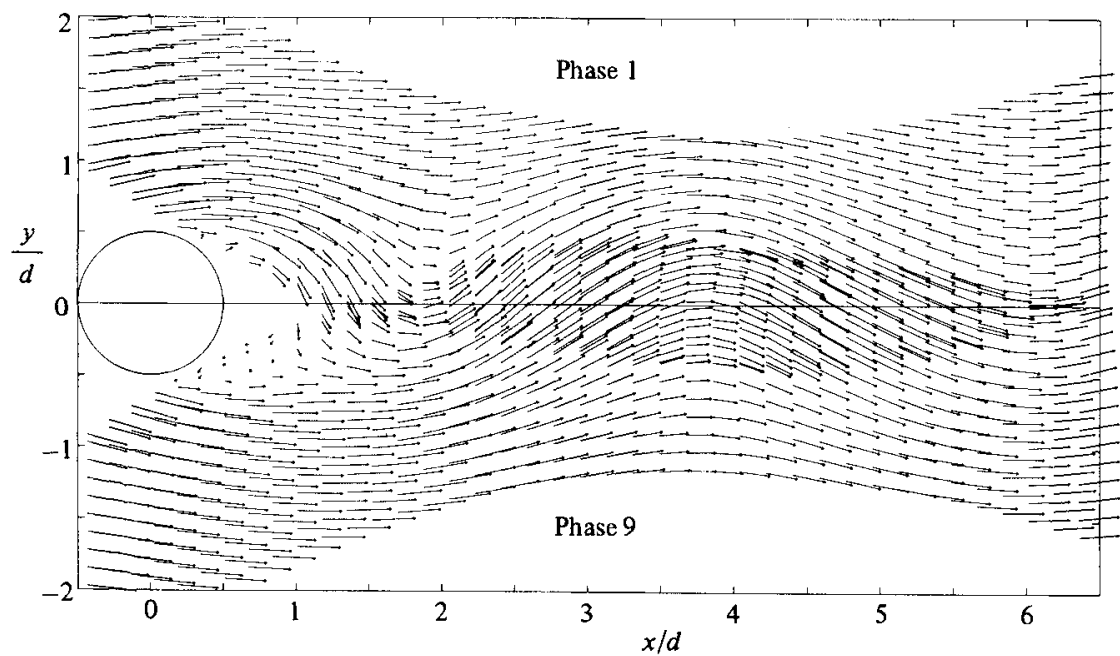

(b)

Figure 12. The velocity field at constant phase $(1,9)$ as viewed from a frame of reference $(a)$ moving downstream at $0.755 u_{\infty},(b)$ fixed with respect to the cylinder.

matches the measured streamwise component of vortex celerity (see table 3) at the particular station $x / d=3.75$. In each figure the cylinder is moving to the left with this same velocity, along with much of the fluid in the base region. The moving observer is displaced between phases by a distance $\Delta x=0.755 u_{\infty}(T / 16)$, where $T=1 / f$ is the shedding period. With $f d / u_{\infty}=0.179$, the distance in question is $\Delta x=0.264 d$ per phase, or $4.22 d$ per cycle. Figures $12(a)-19(a)$ may therefore be interpreted as a sequence of streak photographs taken by a series of moving observers (positioned 0.264 $d$ apart) as each passes the particular station $x / d=3.75$. The length of the arrows is scaled to correspond to an exposure time of about $\frac{2}{3}$ of the interval between phases.

The outstanding kinematical feature associated with entrainment of freestream fluid by the shedding vortices is the formation and evolution of a saddle point in the fluid as seen by an observer moving with the eddies. Evidence of the saddle begins 


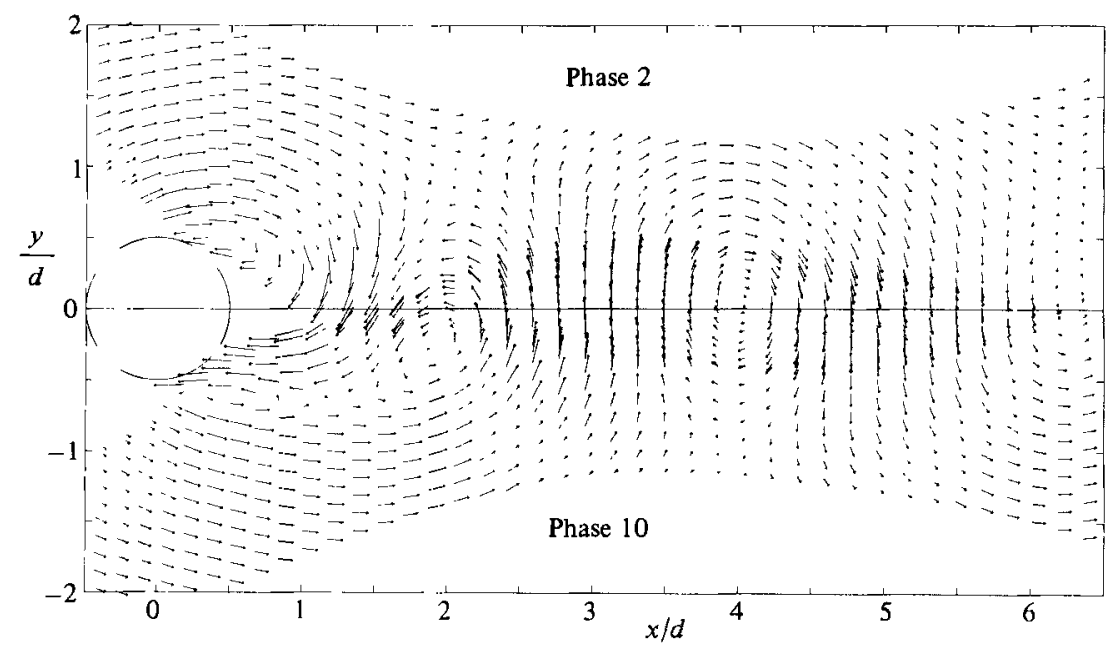

(a)

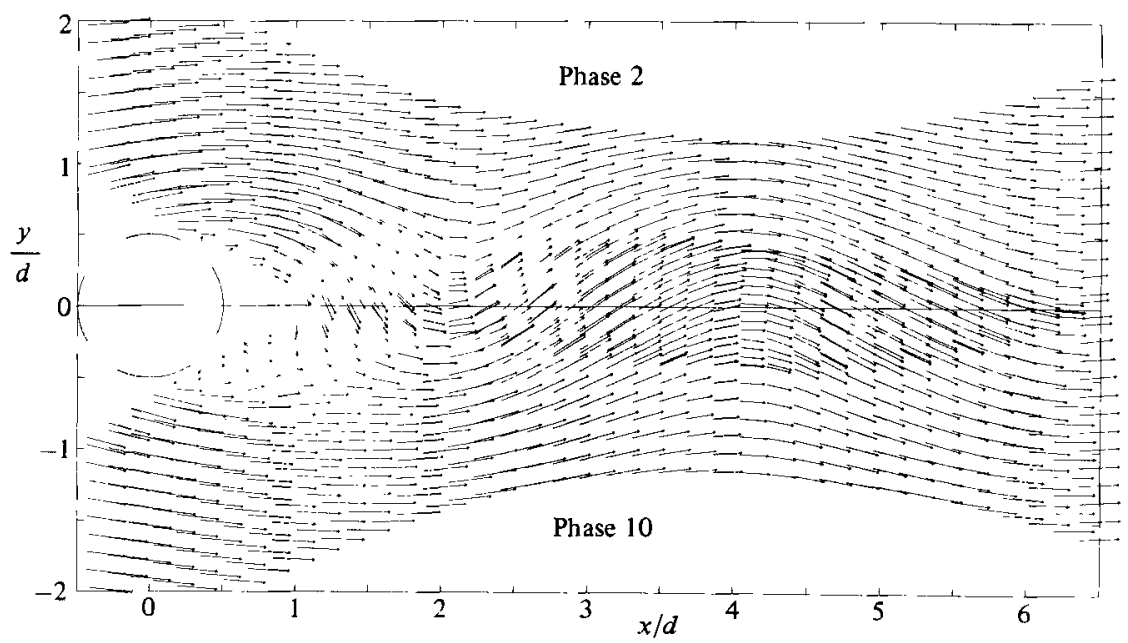

Figure 13. The velocity field at constant phase $(2,10)$ as viewed from a frame of reference (a) moving downstream at $0.755 u_{\infty}$, (b) fixed with respect to the cylinder.

to appear in figure $13(a)(x / d=1.3, y / d=-0.6)$. The saddle continues to develop and by figure $18(a)$ (phase 7,15$)$ has become quite well defined $(x / d=1.7$, $y / d=-0.8$ ). The converging separatrix of the saddle brings in freestream fluid from above and below the cylinder. The diverging separatrix carries this fluid downstream into the vortex with centre at $\bar{x} / d=3.13$, where a process of fluid rollup is occurring, and upstream (referred to the moving observer) into an interaction zone very near the base of the cylinder.

Figures $12(b)-19(b)$ represent the same mean-velocity field at constant phase in a reference frame fixed with respect to the cylinder. The large flow angles, particularly near the plane of symmetry, confirm that a fixed hot-wire $X$-array or a conventional Pitot-static tube would be a very unreliable instrument for this flow.

Figures 12-19 are derived from measurements made on one family of 14 ares which cover only part of the flow field. In subsequent processing, all three families of arcs 


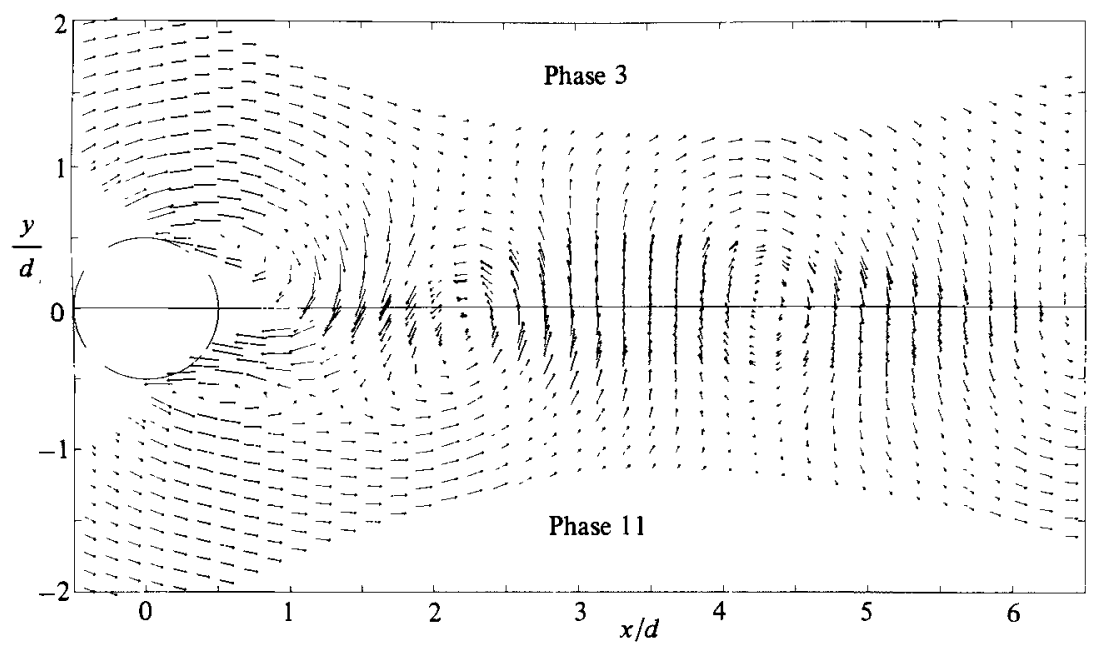

(a)

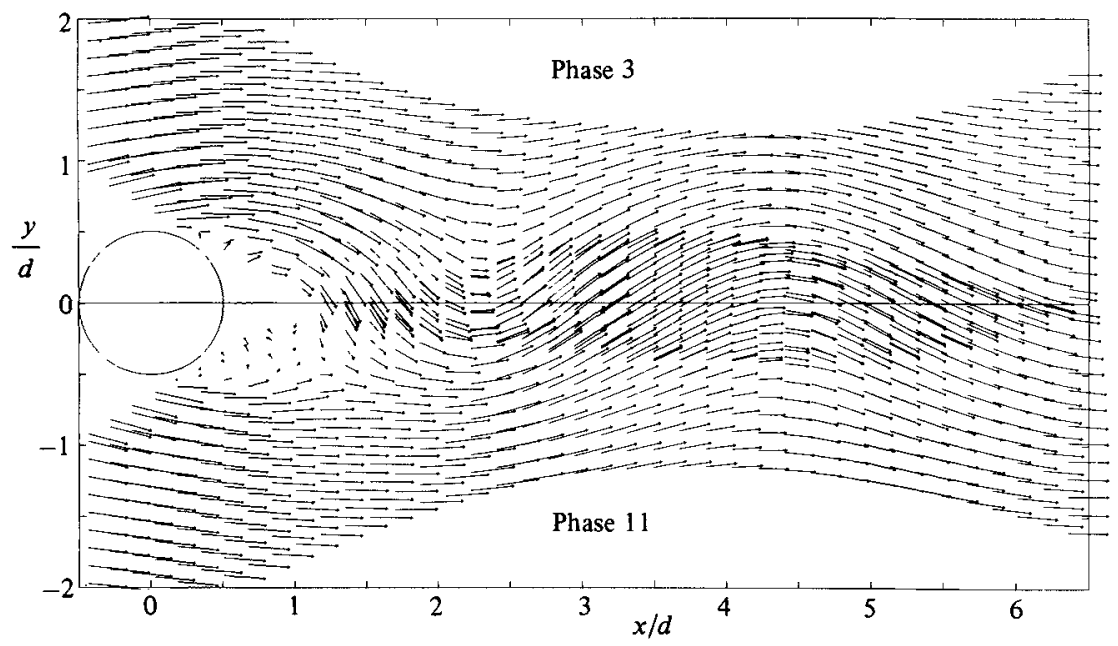

(b)

Figure 14. The velocity field at constant phase $(3,11)$ as viewed from a frame of reference (a) moving downstream at $0.755 u_{\infty}$, (b) fixed with respect to the cylinder.

were used. The data for each variable (without regard to family of origin) were linearly interpolated in $x$ and then in $y$ to obtain values on a grid with nodes spaced $0.1 d$ apart. The data from the two probes were then averaged. Disagreement between the two values for (say) the magnitude of the mean velocity at constant phase was usually less than $0.03 u_{\infty}$, except in the base region, where it could be as much as $0.06 u_{\infty}$. Figures $11(b),(c)$ show typical comparisons for global mean velocity and turbulent shearing stress as measured by the two probes.

At this point the problem of presentation of results becomes most severe. In addition to data for intermittency, velocity and vorticity, data are available for contributions by the periodic and random motions to each Reynolds stress and to the turbulent energy production for 9 phases (including the global mean) - 90 plots in all! Therefore we have chosen to limit the discussion to the results for the mean 


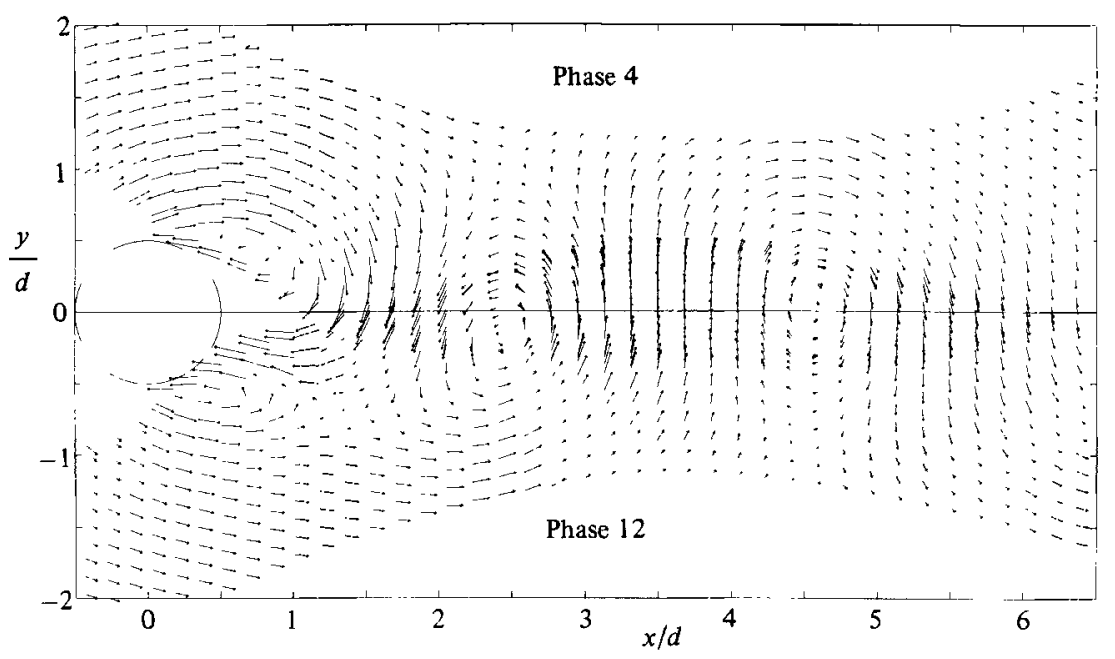

(a)

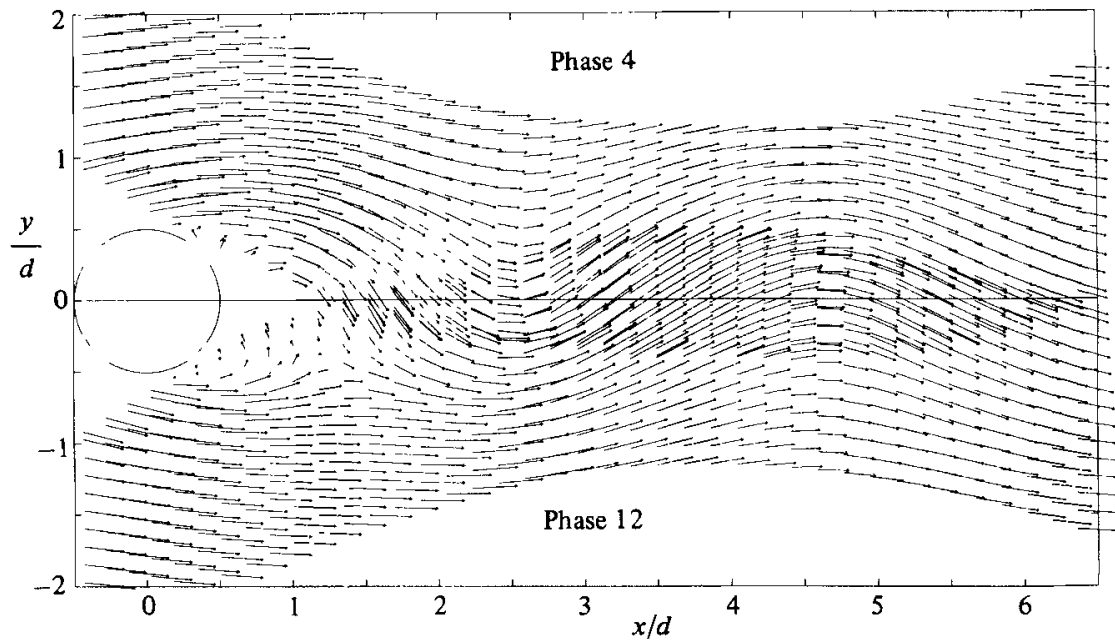

(b)

Figure 15. The velocity field at constant phase $(4,12)$ as viewed from a frame of reference (a) moving downstream at $0.755 u_{\infty}$, (b) fixed with respect to the cylinder.

flow and the flow at one representative phase of the motion (phase 7,15 ). In all cases results are made dimensionless using the freestream velocity and the cylinder diameter, as appropriate, although the normalizing factors may not always be stated explicitly.

\section{Vortex motion}

Interpolated and averaged results for the velocity field at phase $(7,15)$ are shown in figure 20. If the instantaneous flow patterns in this figure and in corresponding figures for other phases are taken at face value, the position of the shed vortices is established for each phase of the motion. However, these patterns should not be taken at face value. The saddles and centres appear at points where the vectors in figures $12(b)-20(b)$ happen to be horizontal and to have the particular magnitude $0.755 u_{\infty}$. If some other 


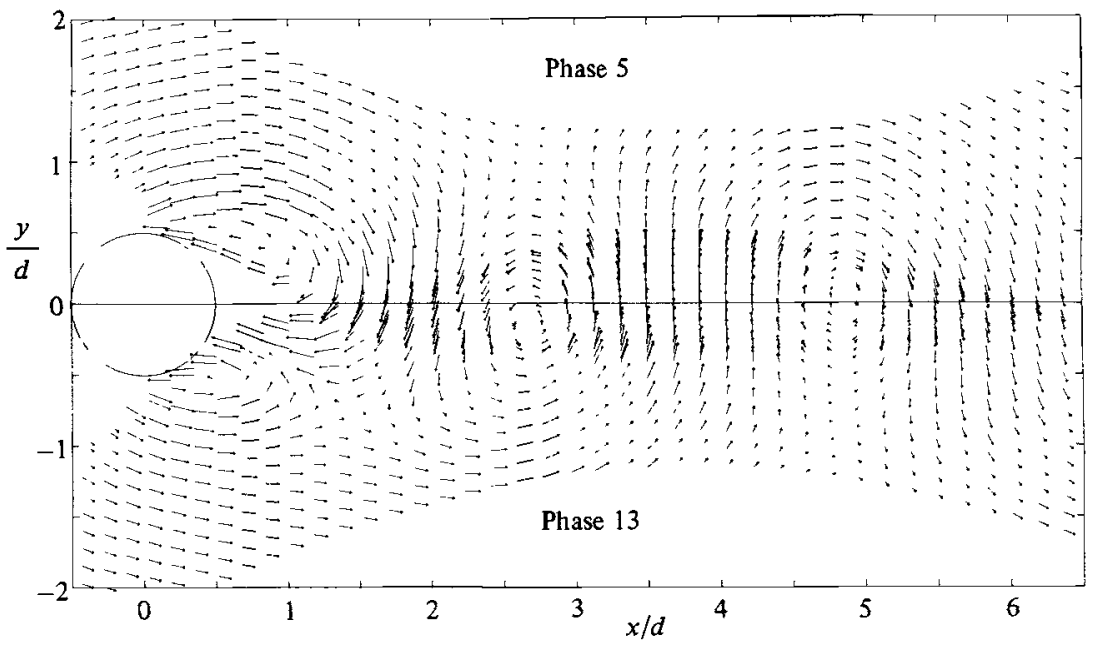

(a)

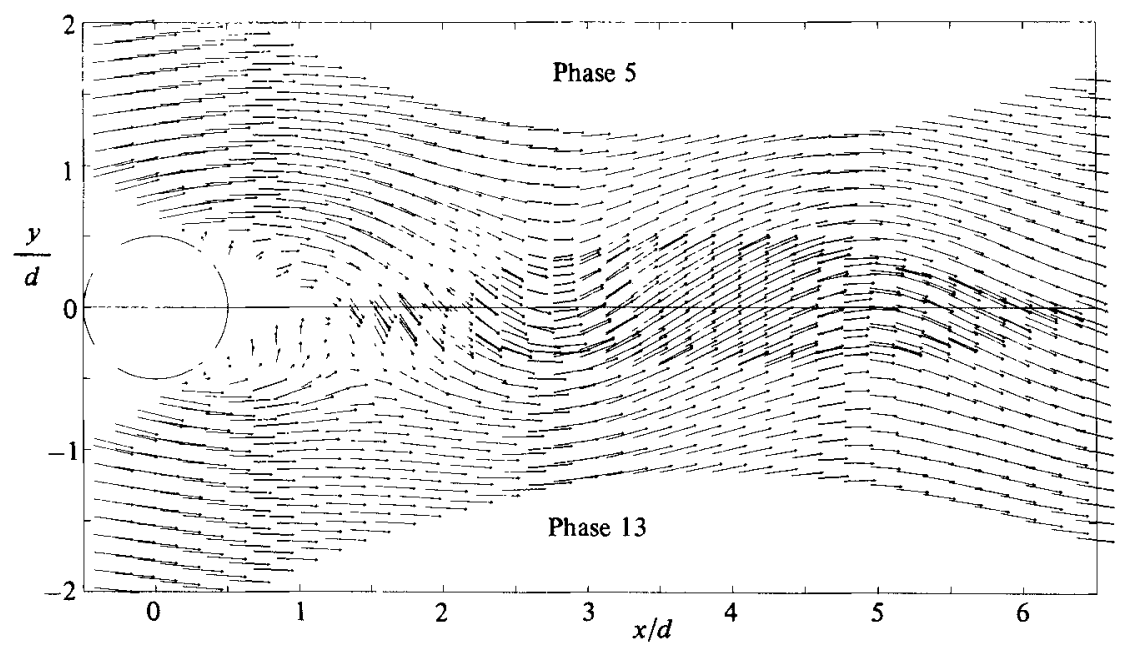

(b)

Figure 16. The velocity field at constant phase $(5,13)$ as viewed from a frame of reference (a) moving downstream at $0.755 u_{\infty},(b)$ fixed with respect to the cylinder.

velocity for the moving observer had been chosen, the centres would be displaced to other locations in the flow. $\dagger$

An unambiguous way to find the vortices is to examine the mean vorticity at constant phase, $\langle\zeta\rangle=\partial\langle v\rangle / \partial x-\partial\langle u\rangle / \partial y$. Taking the curl of the velocity field in figure $20(a)$ or $(b)$ leads to the result for vorticity shown (after one stage of smoothing)

$\dagger$ We did not include a figure showing the instantaneous streamlines in our flow as they would appear to an observer moving at $u_{\infty}$; i.e., as they would be photographed by a stationary camera viewing the wake of a towed body. That the vortices would then appear to lie farther from the plane of symmetry was argued long ago by Hooker (1936). A simple argument emerges from figure $20(b)$, which shows the field $u(x, y), v(x, y)$ at constant $t$ as seen in the laboratory frame. Given a streamwise velocity $c$ for a moving observer, the vortex centres lie at points where $v=0, u=c$. Given that $u$ increases with increasing distance from the wake centreline, Hooker's result follows. 

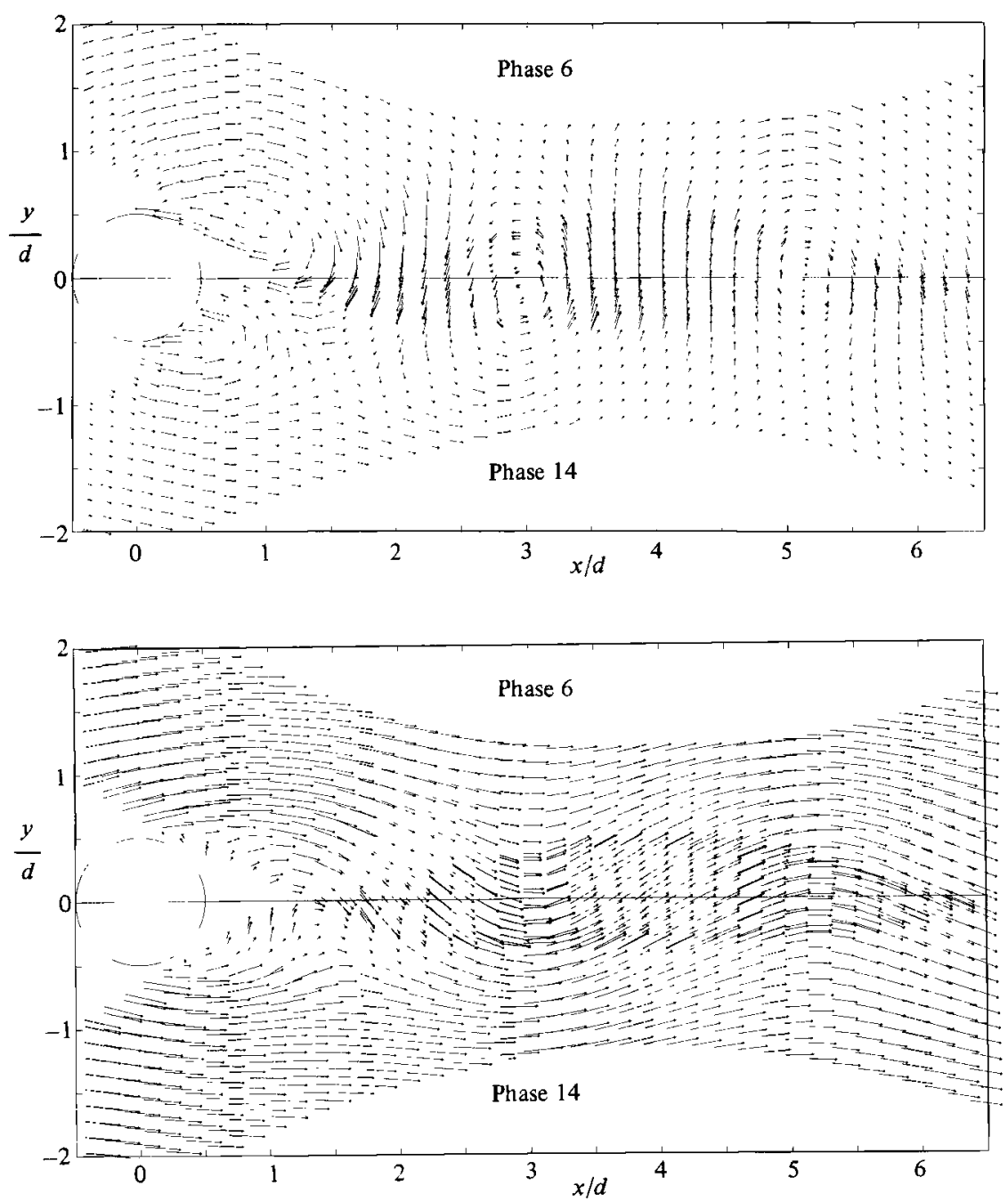

(b)

FIGURE 17. The velocity field at constant phase $(6,14)$ as viewed from a frame of reference (a) moving downstream at $0.755 u_{\infty},(b)$ fixed with respect to the cylinder.

in figure $21(b)$. These data in turn define the vortex circulations and positions through the summation formulas

$$
\begin{gathered}
\frac{\Gamma}{u_{\infty} d}=\sum_{i, j}\left(\frac{\langle\zeta\rangle d}{u_{\infty}}\right)_{i j} \frac{\Delta A}{d^{2}}, \\
\overline{\bar{x}}=\frac{\sum_{i, j}\left(\frac{x}{d}\right)_{i j}\left(\frac{\langle\zeta\rangle d}{u_{\infty}}\right)_{i j} \frac{\Delta A}{d^{2}}}{\frac{\Gamma}{u_{\infty} d}} \\
\frac{\bar{y}}{d}=\frac{\sum_{i, j}\left(\frac{y}{d}\right)_{i j}\left(\frac{\langle\zeta\rangle d}{u_{\infty}}\right)_{i j} \frac{\Delta A}{d^{2}}}{\frac{\Gamma}{u_{\infty} d}},
\end{gathered}
$$




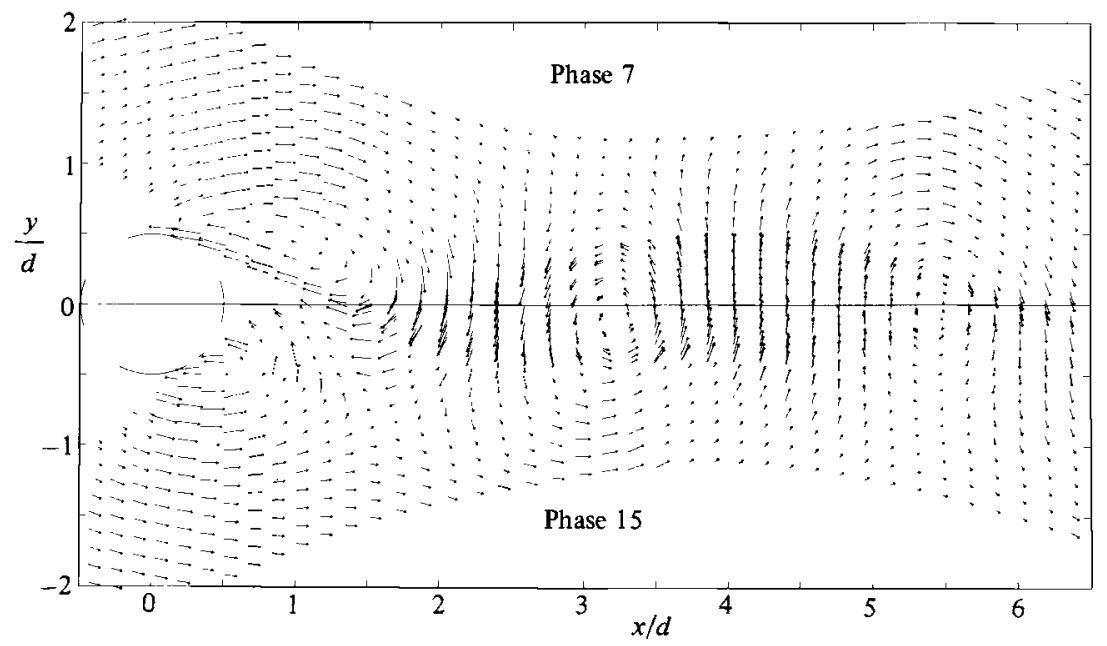

(a)

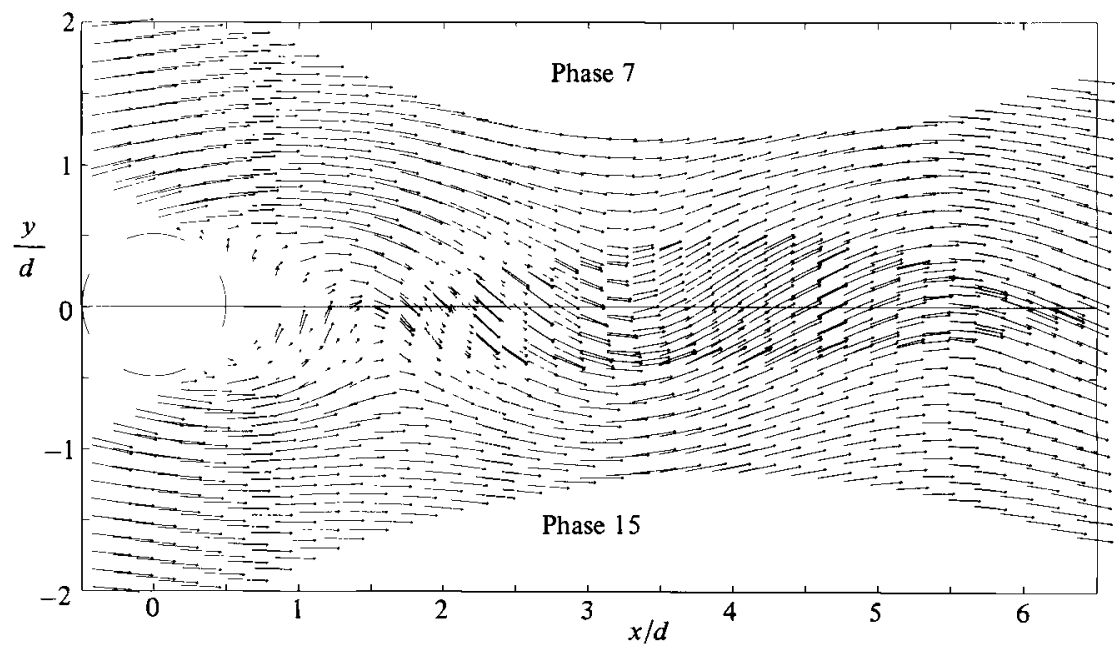

Figure 18. The velocity field at constant phase $(7,15)$ as viewed from a frame of reference $(a)$ moving downstream at $0.755 u_{\infty},(b)$ fixed with respect to the cylinder.

where $\Delta A=0.01 d^{2}$. The summations in each case have been restricted to values of $\left|\langle\zeta\rangle d / u_{\infty}\right|$ greater than 0.03 to avoid relatively noisy data near the outer edges of the vortices, where the vorticity distribution becomes rather flat. $\ddagger$ The coordinates of the centroid of vorticity, the celerity of the centroid, and the circulation are listed in table 3 and plotted as a function of $x / d$ in figure 22 . The data in parentheses in the table are not very meaningful. For phases 13,14 and 15 the region of strong vorticity extends all the way back to the cylinder, without a well-defined neck at which to stop the summations. These were therefore terminated at a rather arbitrary upstream station which varied from $x / d=0.5$ for phase 13 to $x / d=1.5$ for phase

\$ The calculation was also carried out, where feasible, with the threshold set to zero. While the data scatter increased slightly, in no case did the computed values of $\bar{x} / d$ differ significantly from the values in table 3 . Computed values of $\Gamma / u_{\infty} d$ increased by one or two percent. 


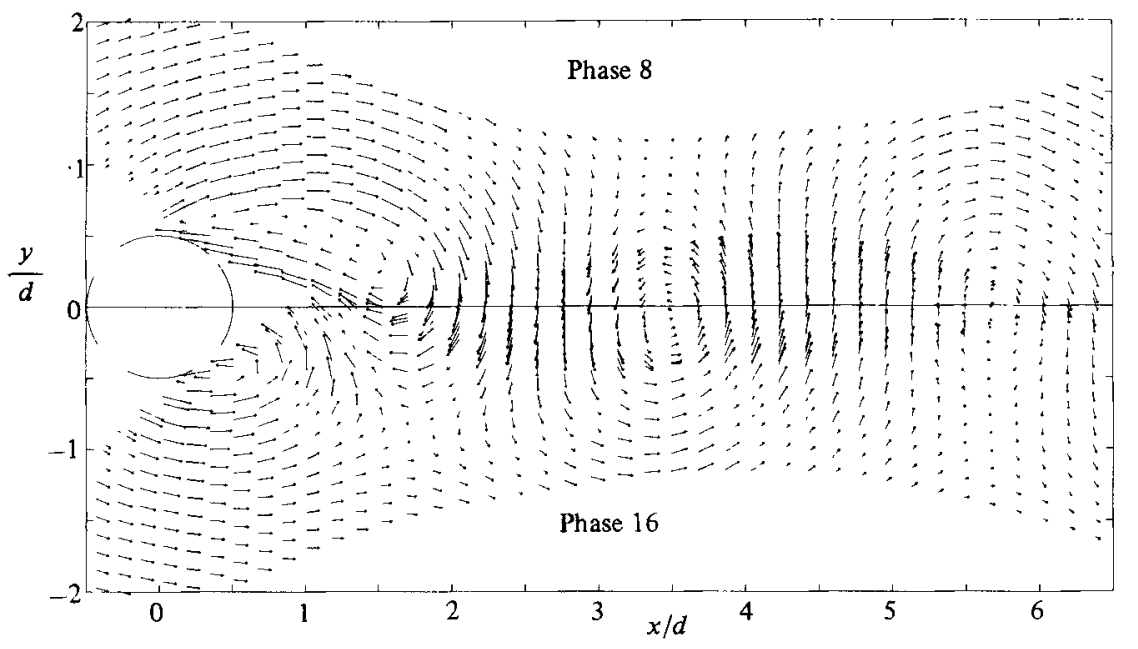

(a)

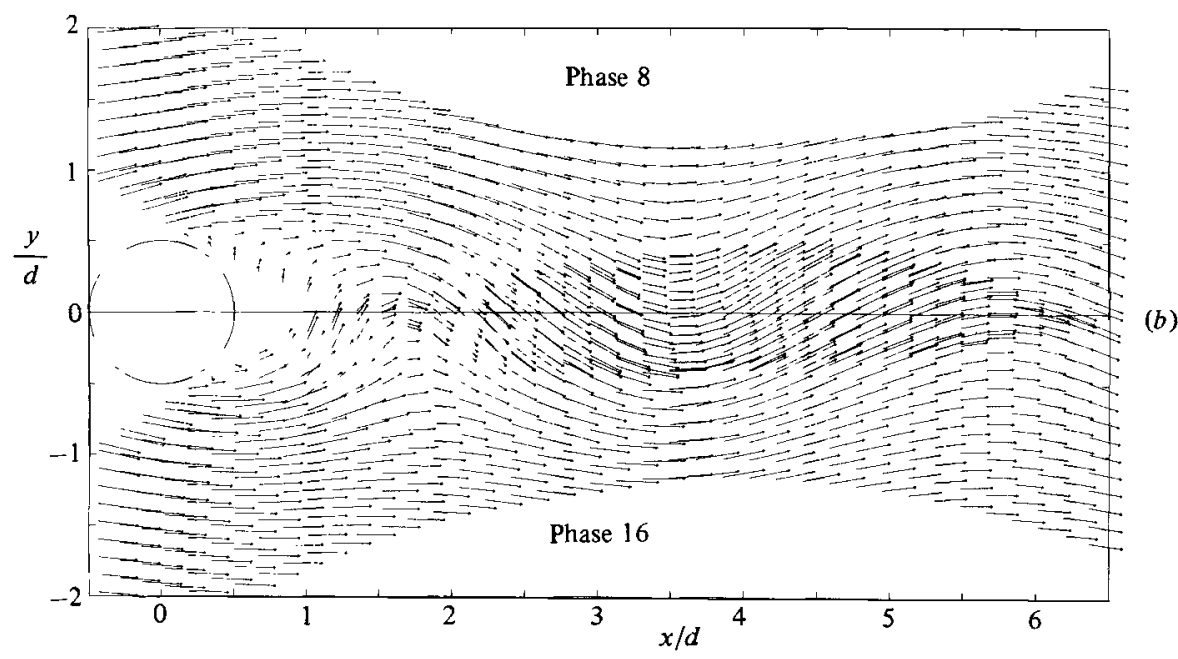

Figure 19. The velocity field at constant phase $(8,16)$ as viewed from a frame of reference (a) moving downstream at $0.755 u_{\infty},(b)$ fixed with respect to the cylinder.

15. For phases 11 and 12 the summations had to be terminated at the last data station $(x / d=8.0)$, although a significant portion of the vortex obviously lay downstream.

Also included in table 3 are the coordinates of points of peak vorticity and the value of the peak vorticity itself, to the accuracy that these quantities could be determined without an elaborate surface-fitting procedure. These data, based on extrema of the mean vorticity, have more range but less authority than data based on integral properties of the vorticity. In particular, the values of peak vorticity may be attenuated by dispersion in vortex trajectory. The values of circulation, on the other hand, should not be seriously affected by dispersion.

The vorticity concentrations for phase $(7,15)$ in figure $21(b)$ are entirely compatible with intermittency data for the same phase, as shown in figure $23(b)$ (this is a contour-plot version of figure 8). For completeness, the intermittency for the global mean flow is included in figure $23(a)$. The fact that neither $\langle\gamma\rangle$ nor $\bar{\gamma}$ reaches unity 
ஓं $\hat{\imath}$

구 के

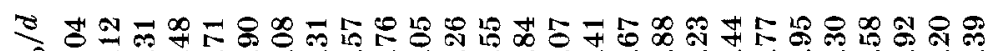
की

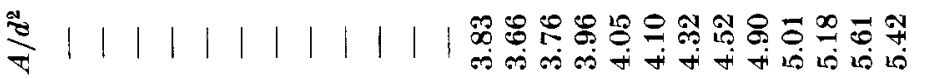

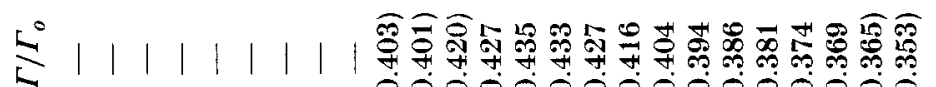

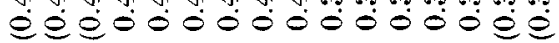

$\frac{乛}{2}$

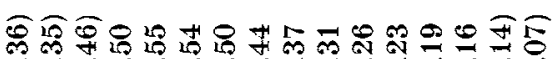
ヘ

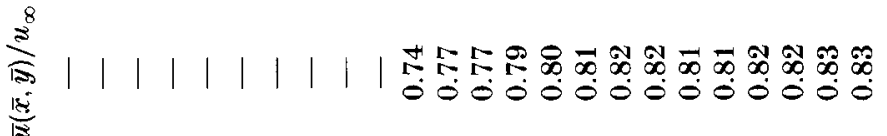

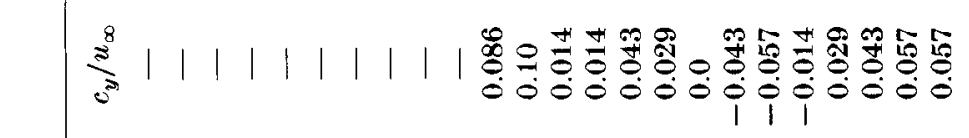

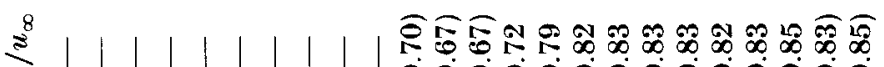
$\dot{\varphi} \dot{\theta} \dot{0} 0 \dot{0} 0 \dot{0} 0 \dot{0} 0 \dot{0} 0 \dot{0}$

$\stackrel{0}{0} 0$

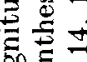

$\checkmark \sim$ 용

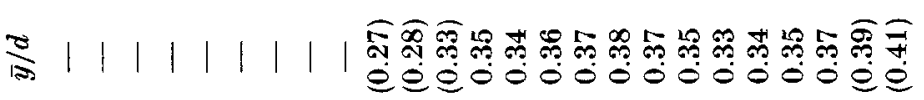

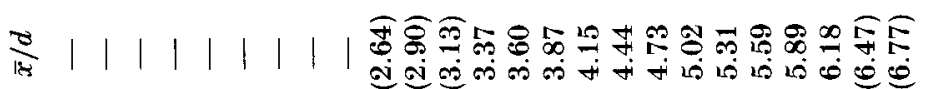

尊 


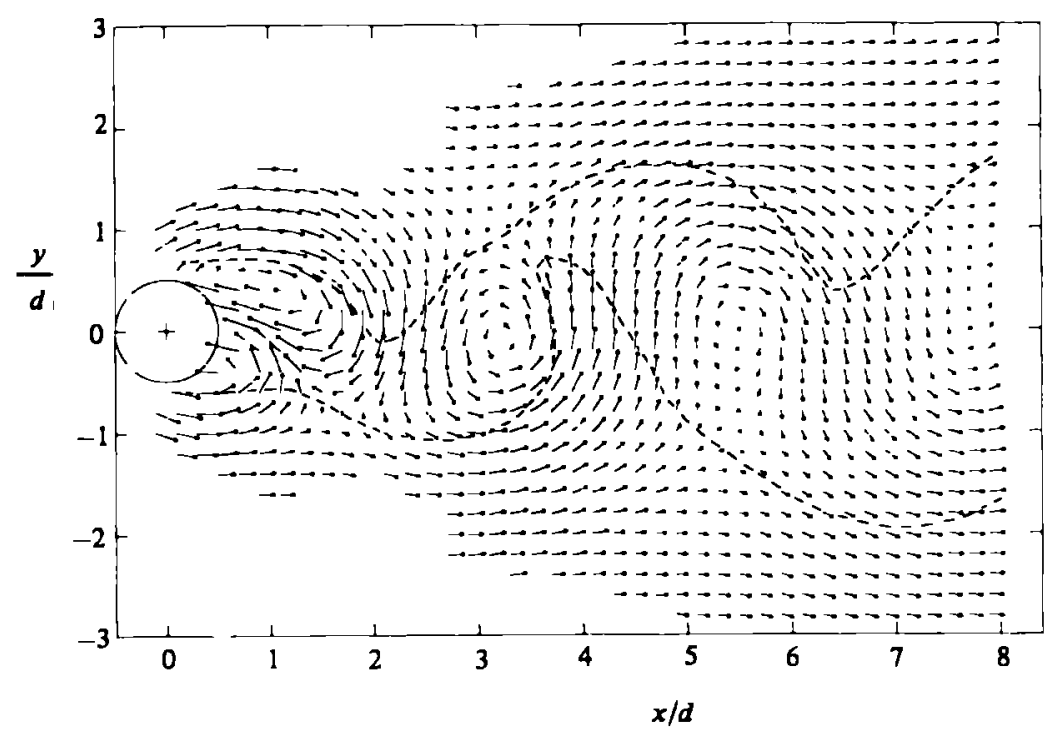

(a)

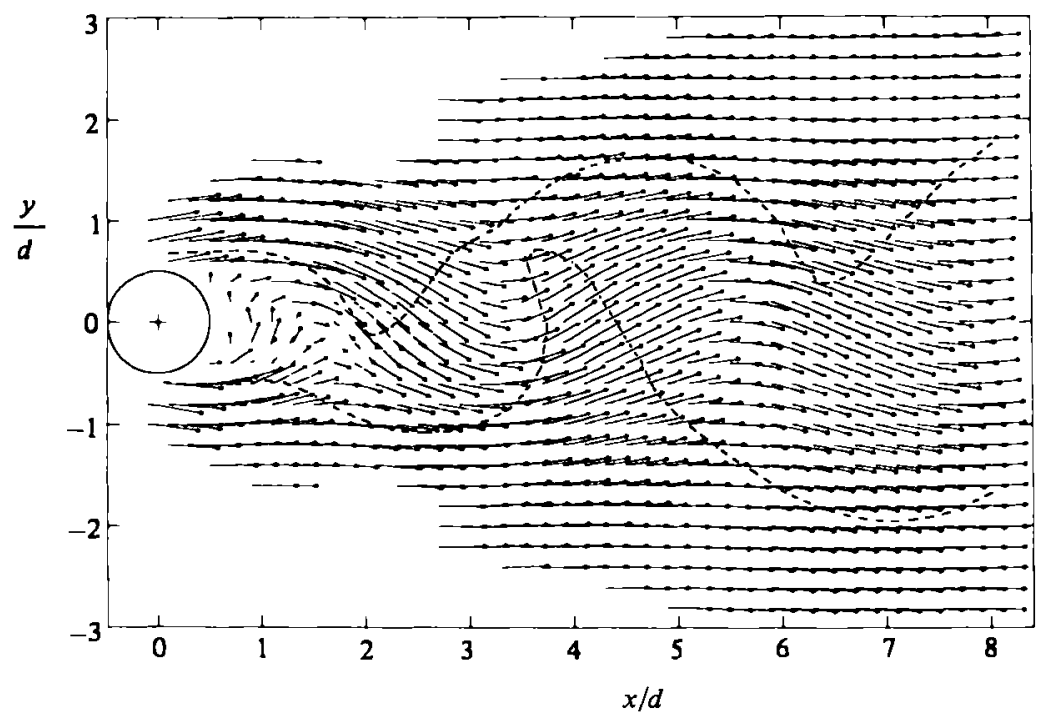

(b)

FIGURE 20. Interpolated velocity field at constant phase $(7,15)$ over 8 diameters of the wake as viewed from a frame of reference $(a)$ moving downstream at $0.755 u_{\infty}$, (b) fixed with respect to the cylinder. Dashed line is contour $\langle\gamma\rangle=0.5$ from figure $23(b)$.

except in the base region has already been pointed out in $\$ 3$. Effects of dispersion are evident in the apparent thickening of the mixing layers near the cylinder, both in figure 23 for intermittency and in figure 21 for vorticity.

Several of the results documented in figures 21-23 and table 3 need comment, either because they agree or because they disagree with results from other studies which have explored the same phenomena.

(a) After they are shed, our vortices move for some distance essentially parallel to and very close to the $x$-axis, with the centroids of vorticity lying roughly $4.3 d$ apart 


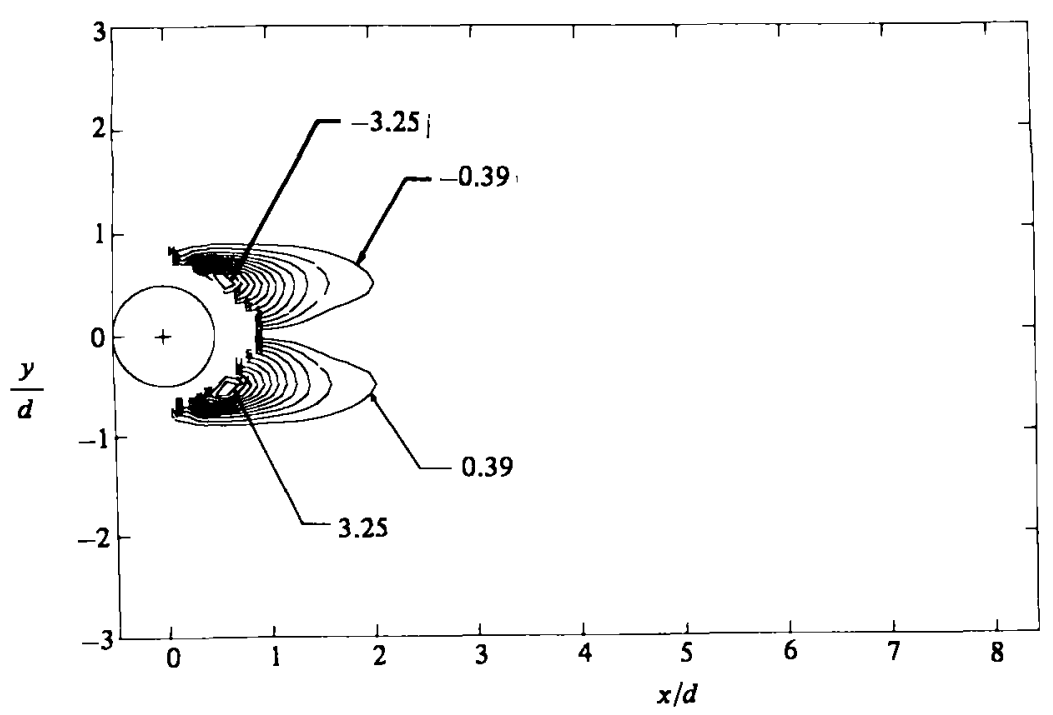

(a)

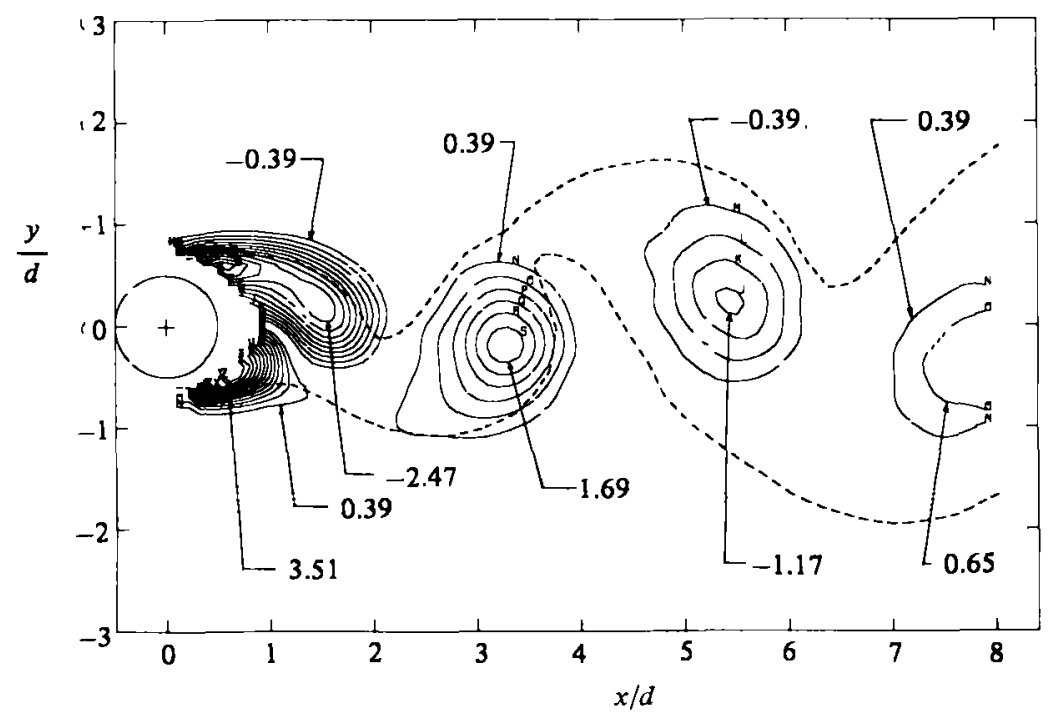

(b)

Figure 21. Vorticity: $(a) \bar{\zeta}$, global mean (contour interval 0.26$) ;(b)\langle\zeta\rangle$, mean at constant phase $(7,15)$ (contour interval 0.26 ). Dashed line is contour $\langle\gamma\rangle=0.50$ from figure $23(b)$.

but lying only about $0.35 d$ from the wake centreline. In this region, therefore, the geometric configuration of the vortex street is quite different from the equilibrium configuration proposed long ago by von Kármán (1912). The celerity inferred from the displacement history of the centroids of vorticity ranges from $0.72 u_{\infty}$ at $x / d=3.6$ to $0.85 u_{\infty}$ at $x / d=5.9$ (cf. the value $0.755 u_{\infty}$ used in plotting figures $12 a-20 a$ ). That the present results are internally consistent is suggested by the fact that the measured celerities are very close to global mean velocities measured at the same positions (cf. the columns $c_{x} / u_{\infty}$ and $\bar{u}(\bar{x}, \bar{y}) / u_{\infty}$ in table 3 ). Correspondence between these two quantities has sometimes been assumed by other investigators in order to infer lateral 


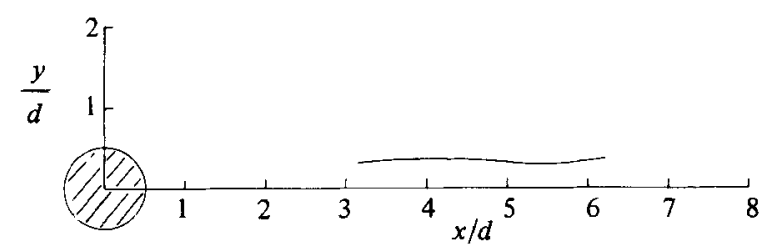

(a)

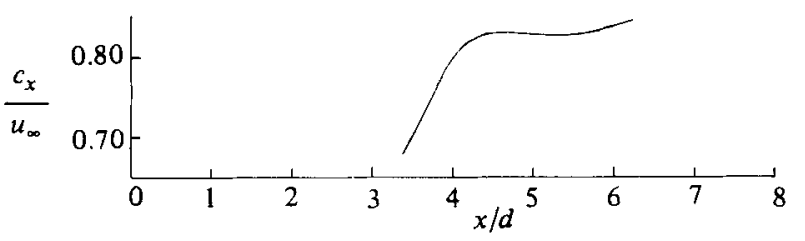

(b)

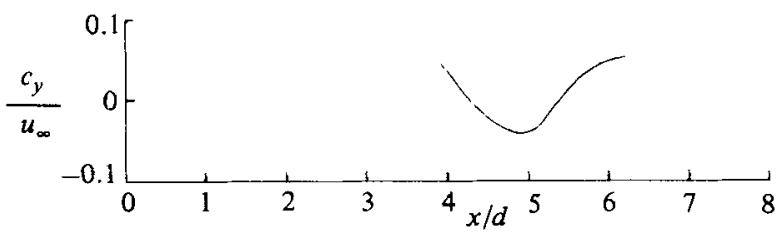

(c)

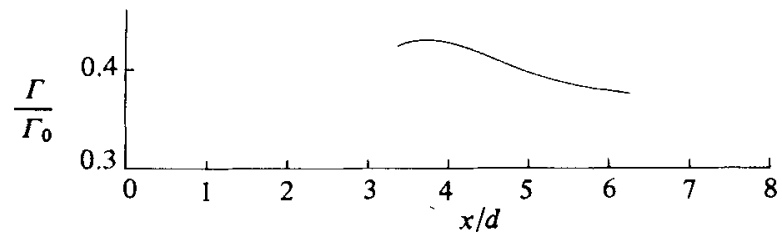

$(d)$

Figure 22. Variation in several vortex parameters (see also table 3): $(a)$ trajectory of the vortex centroid; $(b)$ streamwise vortex celerity; $(c)$ crossflow vortex celerity; $(d)$ vortex circulation.

vortex position when phase velocity is known, but the two quantities have not previously been measured independently.

(b) The measured circulation $\Gamma$ for a vortex in the near wake never exceeds $44 \%$ of the total circulation $\Gamma_{o}$ discharged from one side of the cylinder during a shedding cycle. This shed circulation can be estimated by treating the flow as steady and symmetric (see e.g. Roshko 1954):

$$
\frac{\Gamma_{o}}{u_{\infty} d}=\frac{u_{\mathrm{s}}^{2} \tau}{2 u_{\infty} d}=\frac{1}{2 S t}\left(\frac{u_{\mathrm{s}}}{u_{\infty}}\right)^{2}
$$

where $u_{\mathrm{s}}$ is the velocity just outside the boundary layer at the separation point and $\tau=1 / f$ is the shedding period (two vortices). For the present experiment, $u_{\mathrm{s}}=1.45 u_{\infty}$, and therefore $\Gamma_{o} / u_{\infty} d=5.86$.

Several previous investigators have observed a comparable loss of circulation. If arguments which involve the use of formal analytical models for vortex structure are set aside, there remain at least the experiments by Nielsen (1970) and by Davies (1976). Both authors measured circulation as a line integral of velocity around a closed contour. Davies used hot wires to measure the velocity, while Nielsen used streak photographs of particles in the fluid. In spite of differences in Reynolds number and body shape, as well as some uncertainty about actual vortex position, there is no doubt that typically about half of the available vorticity is lost by cancellation and 


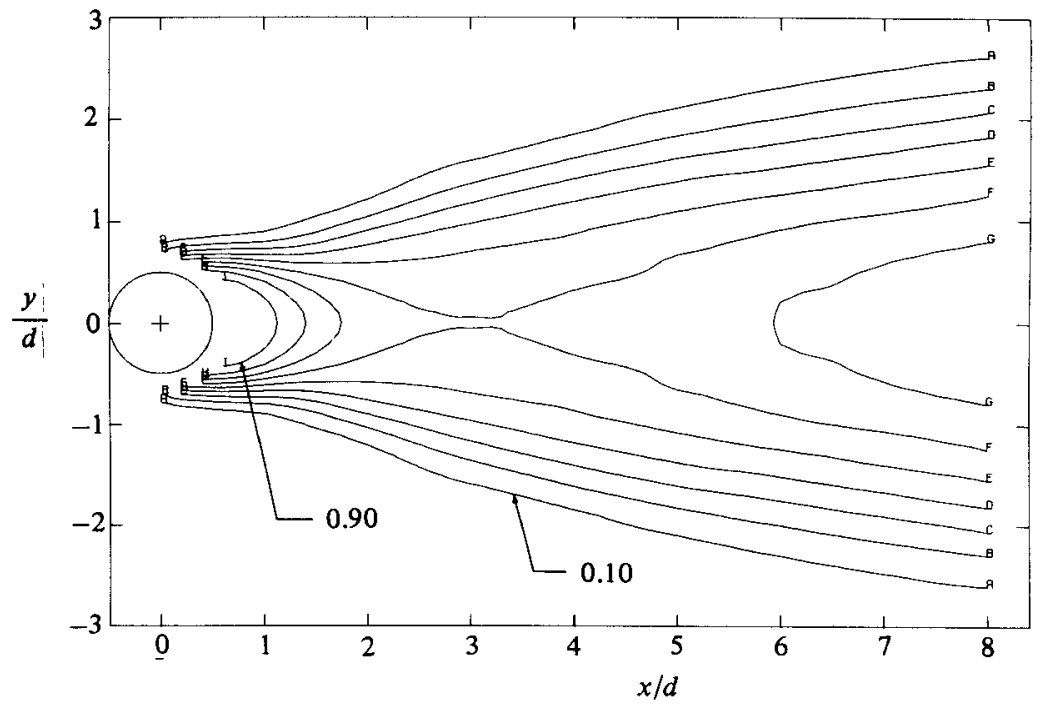

(a)

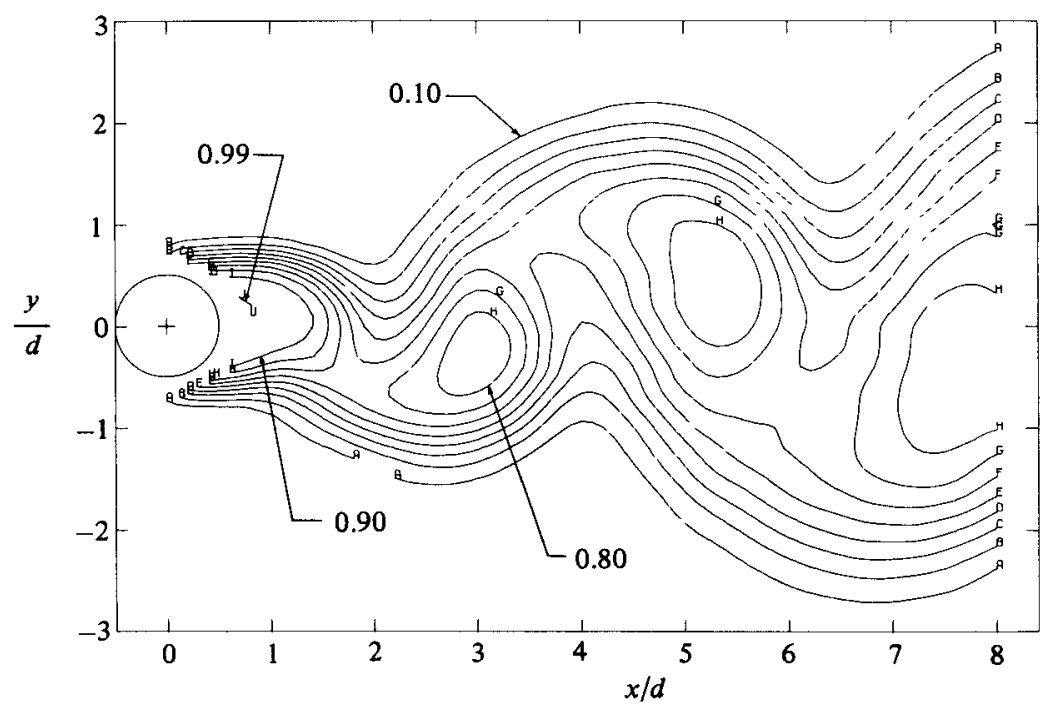

(b)

FIgURE 23. Intermittency: (a) $\bar{\gamma}$, global mean (contour interval 0.10$) ;(b)\langle\gamma\rangle$, mean at constant phase $(7,15)$ (contour interval 0.10 ).

interference in the base region, where there is intense turbulent mixing of vorticitybearing fluid from the two sides of the cylinder (see figures 24-26 below). Nielsen used an ingenious scheme for associating a fixed amount of vorticity with each particle in an attempt to account directly for cancellation in the base flow. A more plausible accounting may eventually emerge from unsymmetrical thermal-tagging schemes like the ones used by Wagner (1976) and by Wlezien \& Way (1979).

(c) The measured circulation in figure $22 d$ reaches a maximum at about $x / d=3.6$ and then decays slowly. We believe that the observed slow decay is real, and that it must be associated with continuous transfer of mean vorticity from one vortex to 
the next through the connecting saddles. Even the direction of this transfer is not obvious, and the present measurements cannot resolve the question. Our guess is that the direction is upstream, because this is the natural sense of the residual transport when two continuous vortex sheets are distorted and stretched to produce a schematic representation of periodic flow in a wake.

The observed rate of decay of circulation in figure $22(d)$ is about $8 \%$ per diameter of vortex travel at $x / d$ near 5 . The rate of decay of peak vorticity $\langle\zeta\rangle_{p}$ (from table 3 ) is about $22 \%$ per diameter. The difference is consistent with the observed growth in vortex area. Let $A$ be defined for convenience as the area over which $\langle\zeta\rangle$ is greater than $10 \%$ of $\langle\zeta\rangle_{\mathrm{p}}$ for a given vortex. The data show that $A$ increases about $14 \%$ per diameter; the combination $\Gamma /\langle\zeta\rangle_{\mathrm{p}} A$ is constant at a value of $0.39 \pm 0.01$ for $x / d$ between 3.6 and 6.2. Nielsen's data, which are for a D-shaped body with rounded edge forward at a relatively low Reynolds number of 4000 , show a maximum value of 0.66 for $\Gamma / \Gamma_{o}$ and a decay rate of about $18 \%$ per diameter of travel near $x / d=6$. Extensive measurements of circulation decay have also been made by Schmidt \& Tilmann (1972) in the near wake of a circular cylinder. The main instrumentation was an ultrasonic beam normal to the plane of the wake, and the measured phase shift was assumed to be a measure of the full circulation integral around a contour enclosing the body. $\dagger$ The observed decay rate for Reynolds numbers between 4000 and 30000 is typically about $8 \%$ per diameter of vortex travel near $x / d=10$, in good agreement with the present result at a higher but still subcritical Reynolds number. Unfortunately, Schmidt \& Tilmann do not report the two quantities needed to evaluate the source strength $\Gamma$ from (11), namely the Strouhal number and the pressure coefficient at separation.

\section{Momentum transport}

The basis for the technique of ensemble averaging at constant phase is that an important component of the flow is periodic in time. In the context of conventional turbulence modelling, the fluctuation away from a global mean $\bar{s}$ includes a contribution $\tilde{s}$ from the periodic large-scale motion and a contribution $s^{\prime}$ from local random or intrinsic local turbulence. Consider a sample population measured at fixed position and fixed phase. The mean variables for this population describe the periodic motion $\tilde{s}$, with the vortices frozen in some average position. Fluctuations away from the mean come from two sources; there are fluctuations due to small-scale random turbulence superimposed on the large-scale vortices, and there are fluctuations due to the fact that the large-scale motion is not itself the same for every vortex; there is dispersion. For the most part, the subsequent discussion will make no distinction, and will refer to all fluctuations $s^{\prime}$ away from the mean at constant phase as being due to random turbulence.

From the point of view of the global mean flow described by (6), Reynolds stresses are associated with both the periodic and the random motions. These stresses are resolved as functions of position and phase by the present measurements. The sign convention in what follows is that positive $\tau_{i j}$ represents transport of positive $i$-momentum in the positive $j$-direction. Hence the algebraic sign of the stresses is opposite to the one usually encountered.

Figures 24(a), 25(a) and 26(a) show the three Reynolds stresses $\tilde{u} \tilde{u}, \tilde{v} \tilde{v}, \tilde{u} \tilde{v}$ at phase

+ The analysis by Schmidt \& Tilmann seems to require that short time intervals occur twice per shedding cycle when there is no appreciable vorticity of either sign in the fluid traversed by the beam. This requirement is not met in the present data for values of $x / d$ less than about 3 , and it also probably cannot be met at large distances downstream of the body. 


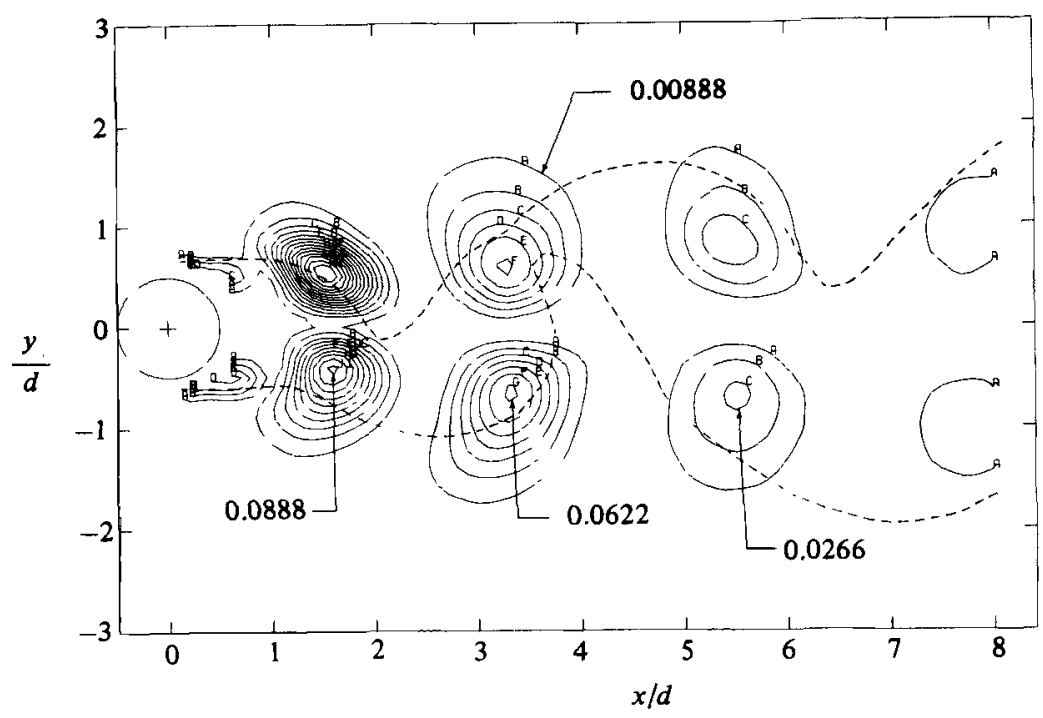

(a)

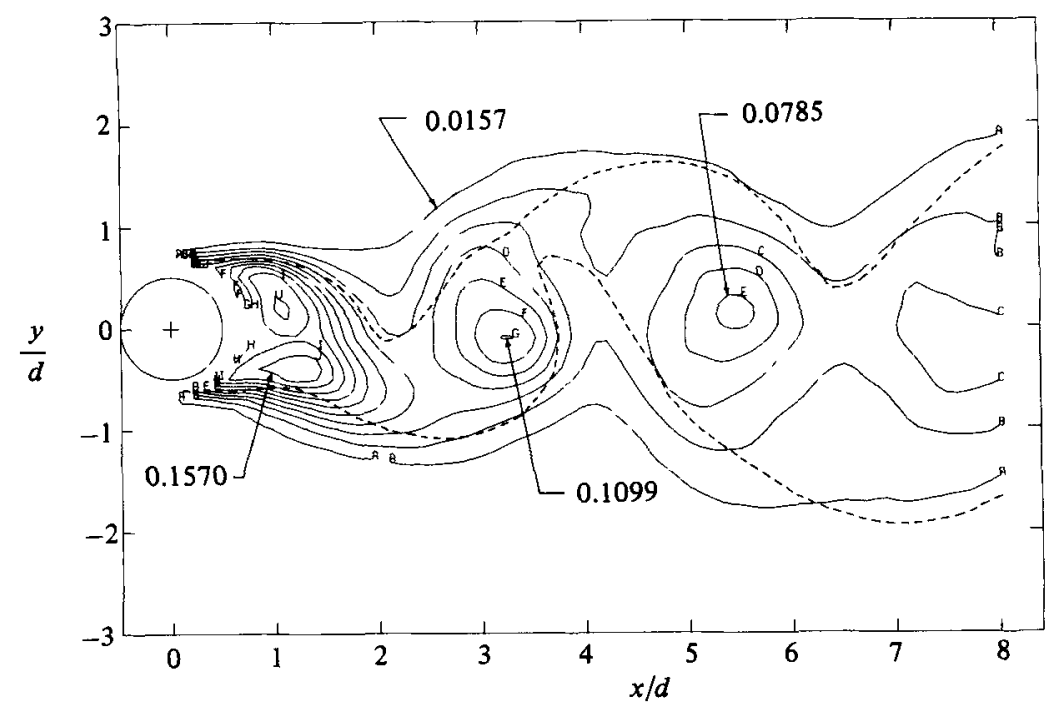

(b)

Figure 24. Contours for streamwise mean Reynolds normal stress at constant phase $(7,15):(a)$ $\tilde{u} \tilde{u} / u_{\infty}^{2}$ (contour interval 0.00888 ); $(b)\left\langle u^{\prime} u^{\prime}\right\rangle / u_{\infty}^{2}$ (contour interval 0.0157). Dashed line is contour $\langle\gamma\rangle=0.5$

$(7,15)$ for the periodic motion. Superposition of these figures on the complete intermittency plot in figure $23(b)$ and on the vorticity plot in figure $21(b)$ shows that the range of the periodic stresses extends well outside the turbulent region. The stress patterns show in all cases a strong symmetry with respect to the wake centreline and a remarkable indifference to the local presence or absence of turbulence. The motion is essentially a local rotation (with respect to the global mean) which generates peaks in $\tilde{u} \tilde{u}$ in figure $24(a)$ above and below each vortex, with $\tilde{u}$ positive for one peak and negative for the other. Similarly, alternating peaks in $\tilde{v} \tilde{v}$ in figure $25(a)$ correspond to positive and negative peaks in $\tilde{v}$. The labelled mean-square contours $\tilde{v} \tilde{v}=0.371$, 


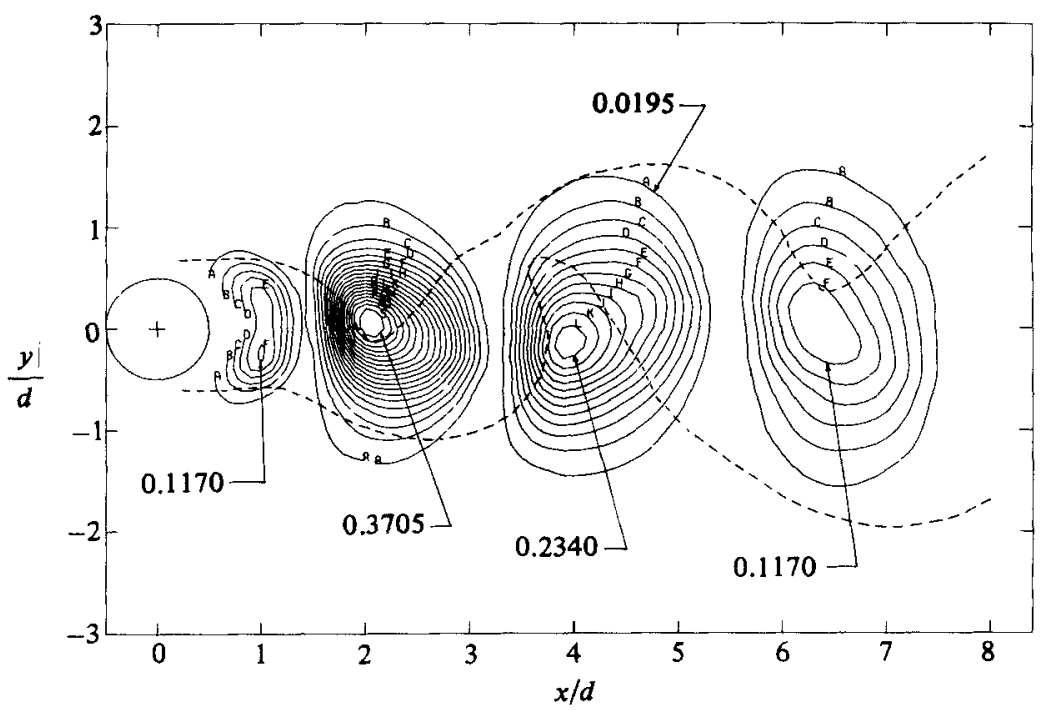

(a)

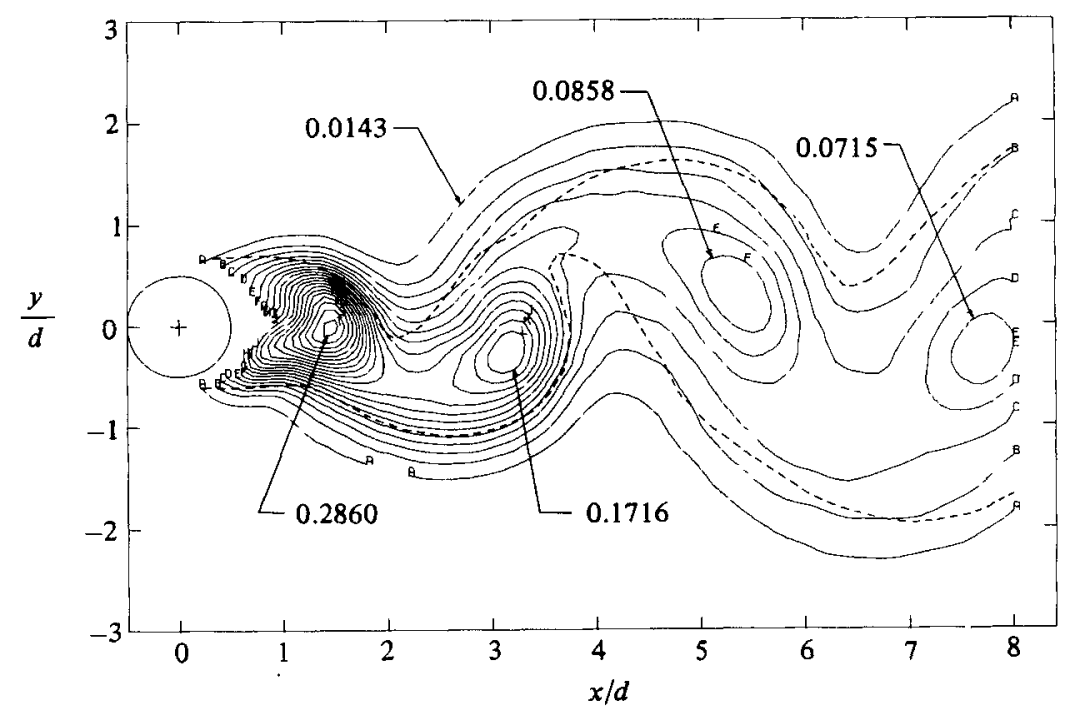

(b)

FigURe 25. Contours for crossflow mean Reynolds normal stress at constant phase $(7,15):(a) \tilde{v} \tilde{v} / u_{\infty}^{2}$ (contour interval 0.0195); $(b)\left\langle v^{\prime} v^{\prime}\right\rangle / u_{\infty}^{2}$ (contour interval 0.0143). Dashed line is contour $\langle\gamma\rangle=0.5$.

$0.234,0.117$ at $x / d=2.1,4.0,6.4$ in the latter figure imply peak root-mean-square values of $\tilde{v}=0.609,0.484,0.342$ respectively. Induced vertical velocities in the plane of symmetry of the near wake are indeed formidable.

One finding of the present research is that the vortex centres in the near wake lie unexpectedly close to the plane of symmetry, at least in the first few diameters of the wake. One consequence is that the quantity $\tilde{u} \tilde{u}$ in figure $24(a)$ is very nearly zero on the plane of symmetry throughout the shedding cycle, and therefore for the global mean flow as well (ef. $\overline{\tilde{u} \tilde{u}}$ in figure $27 a$ ). Given a signal from a single fixed hot wire in the plane of symmetry, the component of this signal at twice the Strouhal frequency would have to come not from the $\tilde{u}$-component but from rectification of the $\tilde{v}$-component associated with deep incursions of freestream fluid. 


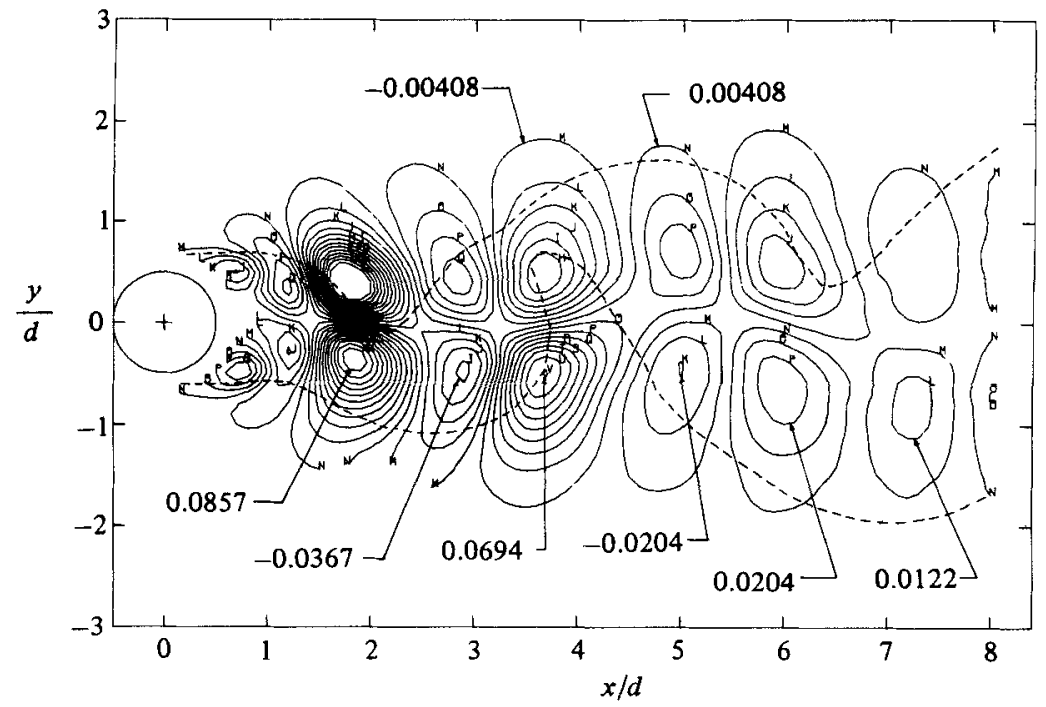

(a)

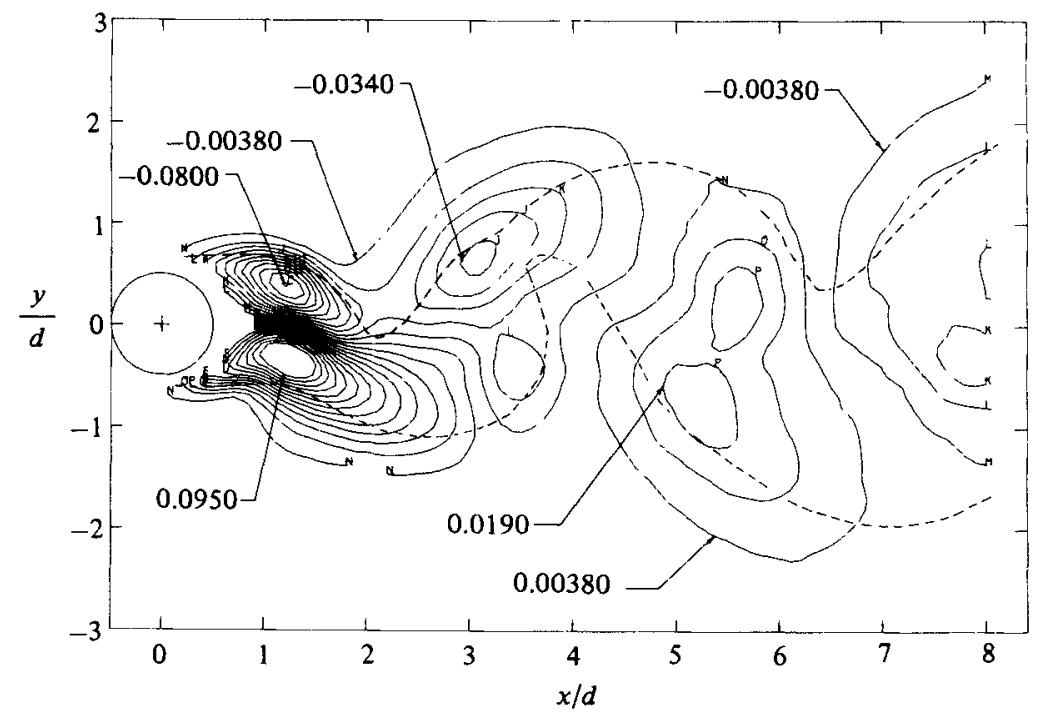

(b)

Figure 26. Contours for mean Reynolds shearing stress at constant phase $(7,15):(a) \tilde{u} \tilde{v} / u_{\infty}^{2}$ (contour interval 0.00816); $(b)\left\langle u^{\prime} v^{\prime}\right\rangle / u_{\infty}^{2}$ (contour interval 0.00760). Dashed line is contour $\langle\gamma\rangle=0.5$.

Figure 24(b) shows the random streamwise fluctuations $\left\langle u^{\prime} u^{\prime}\right\rangle$ at phase $(7,15)$. The figure shows a series of peaks corresponding to the vortex centres with connecting ridges in between them. Note that the fluctuation levels due to the random turbulence are appreciably larger than the fluctuation levels due to large-scale periodic motions; it is understandable that the periodic component might not be very conspicuous in an unprocessed signal from a stationary probe. Figure $25(b)$ shows $\left\langle v^{\prime} v^{\prime}\right\rangle$ behaving in much the same way as $\left\langle u^{\prime} u^{\prime}\right\rangle$ with a somewhat larger amplitude and with the same peaks and ridges.

Figures 24 and 25 contain some indirect evidence that phase jitter arising from 
irregularity in the vortex shedding has not seriously affected the measurement of Reynolds stresses due to random turbulence. If such effects were important, one might expect to see local maxima in the random stresses at places where large gradients in $\tilde{u} \tilde{u}$ and $\tilde{v} \tilde{v}$ occur. These would be particularly evident outside the boundary $\langle\gamma\rangle=0.5$, where the random stresses would be expected to be small. For example, at $x / d=1.5, y / d=1.0$ in figure $24(b)$, the quantity $\left\langle u^{\prime} u^{\prime}\right\rangle$ shows no evidence of the large gradient in $\tilde{u} \tilde{u}$ which appears at the same location in figure $24(a)$. We conclude that the technique used for averaging the flow at constant phase was fairly effective in separating the two phenomena.

Figure 26 shows the two contributions to the Reynolds shearing stress. The stress associated with the periodic motion in figure $26(a)$ exhibits consistent antisymmetry about the wake centreline; regions of strong $x$-momentum flux toward the wake centreline are separated by smaller regions of weaker $x$-momentum flux away from the centreline. The most interesting feature of figure $26(a)$ is the relatively large stress associated with deep incursions of freestream fluid into the regions between the vortices. In fact, the global averages of all three stress components must contain significant contributions from periodic motions of freestream fluid (cf. figures 27-29).

The contribution to the shearing stress by the random turbulence is shown in figure $26(b)$. As in the previous two figures, the stress levels are comparable to the stresses associated with the large-scale periodic motions. However, there is one important difference. Whereas the normal stresses due to the random turbulence have a maximum near each vortex centre, the shearing stress due to the random turbulence has an extreme value near the saddle between the vortices. This conclusion can be readily verified by superposing figures $26(b)$ and $20(a)$. The sign of the shearing stress is such as to produce a rapid flux of $x$-momentum away from the freestream and toward the interior of the turbulent region.

The data displayed in figures $24(b), 25(b)$ and $26(b)$ can be used to compute the correlation coefficient $R$ for the random turbulence, where by definition

$$
R=\frac{\left\langle u^{\prime} v^{\prime}\right\rangle}{\left\langle u^{\prime} u^{\prime}\right\rangle^{\frac{1}{2}}\left\langle v^{\prime} v^{\prime}\right\rangle^{\frac{1}{2}}}
$$

The value obtained at the vortex centre located near $\bar{x} / d=3.13$ is $R=-0.08$, compared with $R=-0.50$ at the saddle just above it. For the centre and saddle near $\bar{x} / d=5.6$, the corresponding values of $R$ are 0.22 and 0.46 respectively. It is clear that the random turbulence is strongly coupled to the strain and vorticity fields associated with the large eddies.

Globally averaged Reynolds stresses for the periodic and random components of the motion are shown in figures 27, 28 and 29 . According to (6), the stresses of interest in turbulence modelling (given that the flow is treated as statistically stationary) are obtained by adding the two components $(a)$ and $(b)$ in each figure. The two components are themselves an average over one shedding cycle of measurements like those in figures 24,25 and 26.

In figure 27 both components of the streamwise normal stress exhibit double peaks up to the end of the vortex-formation region, but thereafter the random turbulence exhibits only a single peak. The component due to the large-scale periodic motion remains bimodal throughout the near wake and makes virtually no contribution on the centreline. The two components of cross-stream normal stress are shown in figure 28 . The similarity between figures $28 a$ and $(b)$ is remarkable in view of the complete dissimilarity between the motions which produce the stresses (cf. figures $25 a, b$ ). The two components of the globally averaged shearing stress are shown in figure 29 . 


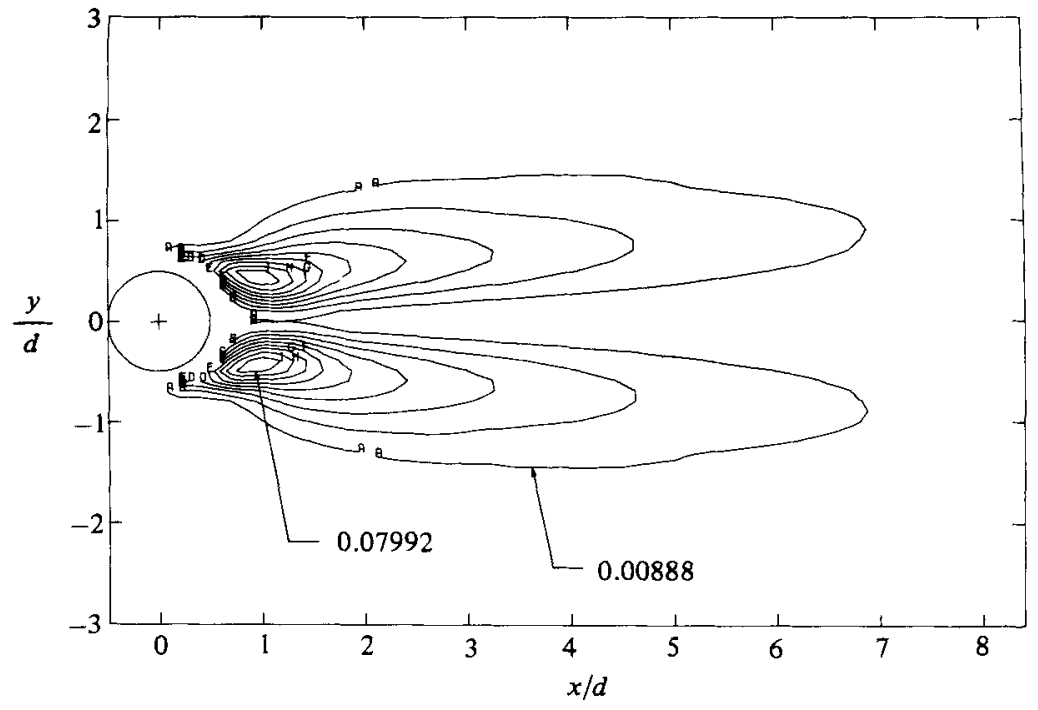

(a)

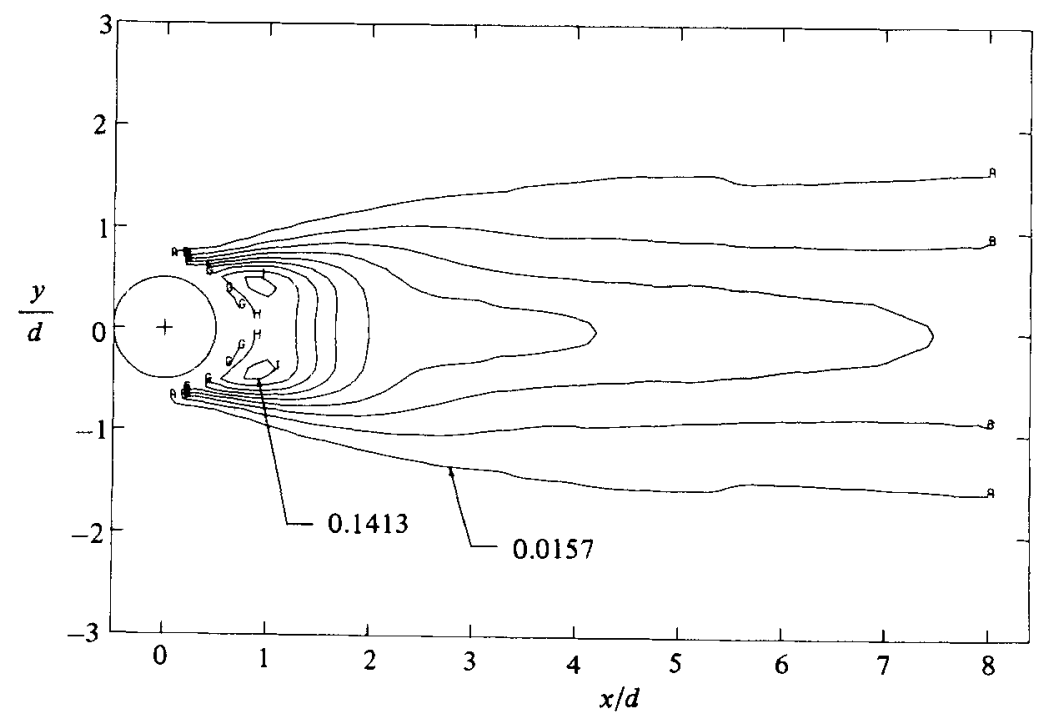

(b)

Figure 27. Contours for global mean streamwise Reynolds normal stress: $(a) \overline{\tilde{u}} \bar{u} / u_{\infty}^{2}$ (contour

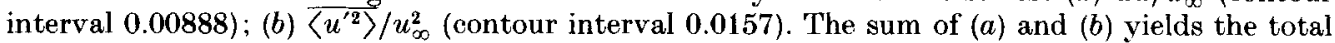
Reynolds stress.

Again, the similarities between figures $29(a)$ and $(b)$ are remarkable in view of the dissimilarities between figures $26(a)$ and $(b)$.

Both components of all three globally averaged stresses reach their extreme values near the end of the vortex formation region, at roughly $x / d=1.0-1.5$, and then decay rapidly with increasing values of $x$. The large stresses inside the formation region account for the small size of the mean separation bubble and the rapid decay of the wake defect velocity. For example, if we add together the two components of the shearing stress in figure 29 at $x / d=1.1, y / d=-0.5$, we obtain $\overline{\tilde{v} \tilde{v}}+\overline{\left\langle u^{\prime} v^{\prime}\right\rangle}=0.12$, 


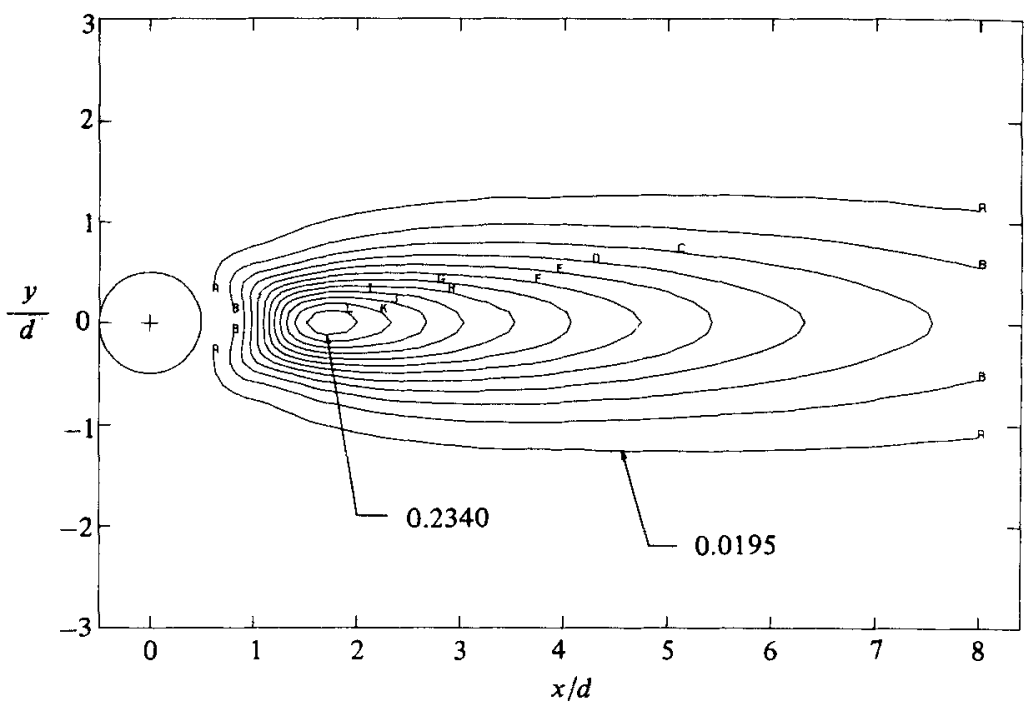

(a)

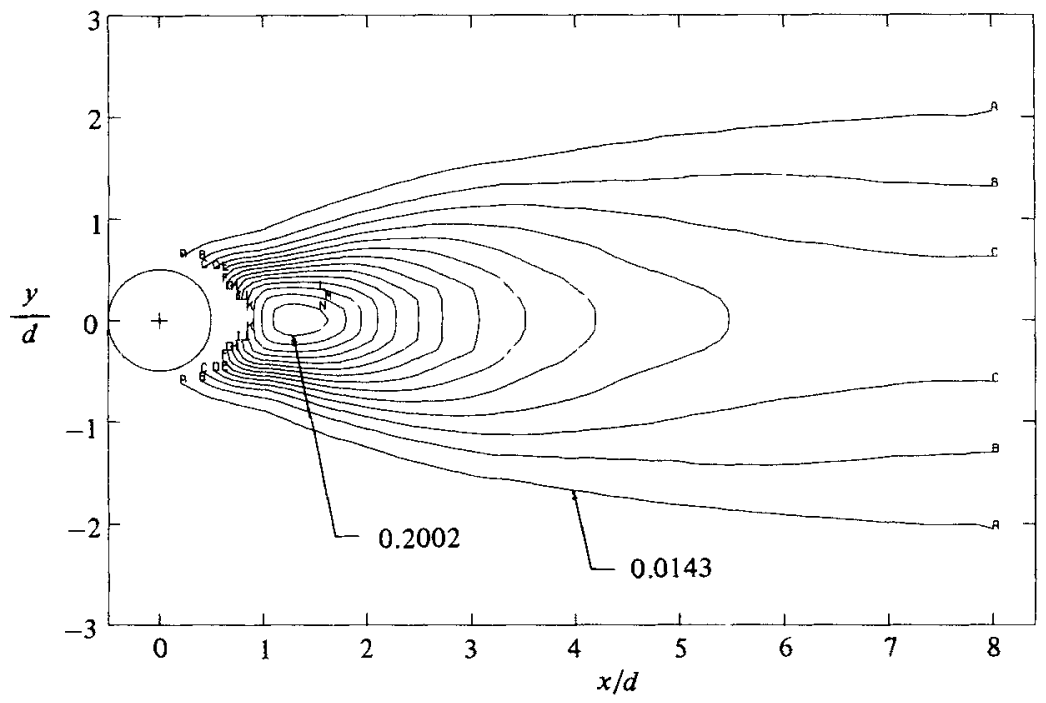

(b)

Frgure 28. Contours for global mean crossflow Reynolds normal stress: $(a) \overline{\tilde{v}} \overline{\tilde{v}} / u_{\infty}^{2}$ (contour interval $0.0195) ;(b) \overline{\left\langle v^{\prime 2}\right\rangle} / u_{\infty}^{2}$ (contour interval 0.0143). The sum of $(a)$ and $(b)$ yields the total Reynolds stress.

a value roughly one order of magnitude larger than the maximum shearing stress in a turbulent plane mixing layer.

\section{Discussion}

\section{Topology and entrainment}

Several authors have recently considered the problem of defining the topology of the near wake in plane flow. They include Peake \& Tobak (1980), whose figure 30 refers to an analogous steady flow downstream of a slender body at angle of attack, Coles 
Entrainment and transport in the near wake of a circular cylinder

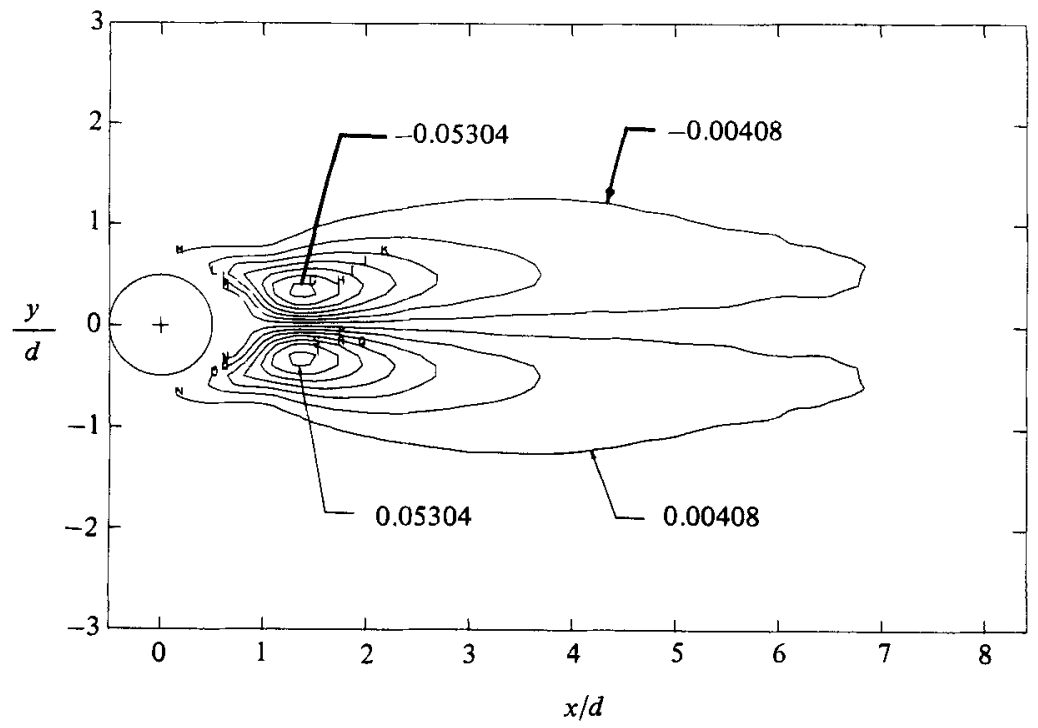

(a)

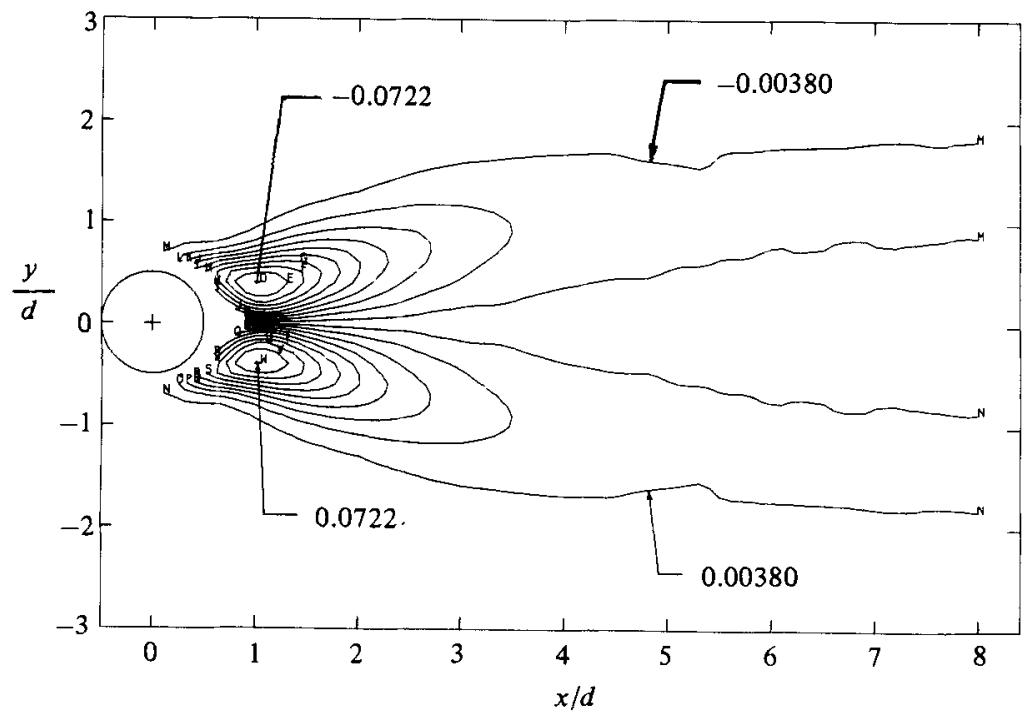

(b)

Figure 29. Contours for global mean Reynolds shearing stress. (a) $\overline{\tilde{u} \tilde{v}} / u_{\infty}^{2}$ (contour interval 0.00816); (b) $\overline{\left\langle u^{\prime} v^{\prime}\right\rangle} / u_{\infty}^{2}$ (contour interval 0.00760 ). The sum of $(a)$ and $(b)$ yields the total Reynolds stress.

(1981) and Perry, Chong \& Lim (1982). For the case of laminar flow, some valuable flow visualization is also available in the work of Zdravkovich (1969) and Matsui (1981).

Our own contribution to the subject is presented in figure 30 , in which the eight sketches are intended to correspond roughly to the eight phases of our experimental data. The material used to develop the figure includes figure $20(a)$ for mean velocity, figure $21(b)$ for mean vorticity, and figure $23(b)$ for intermittency, together with similar figures for other phases. We were also glad to have the early measurements by Drescher (1956) of the pressure distribution at constant phase on a eircular cylinder at subcritical Reynolds number. 


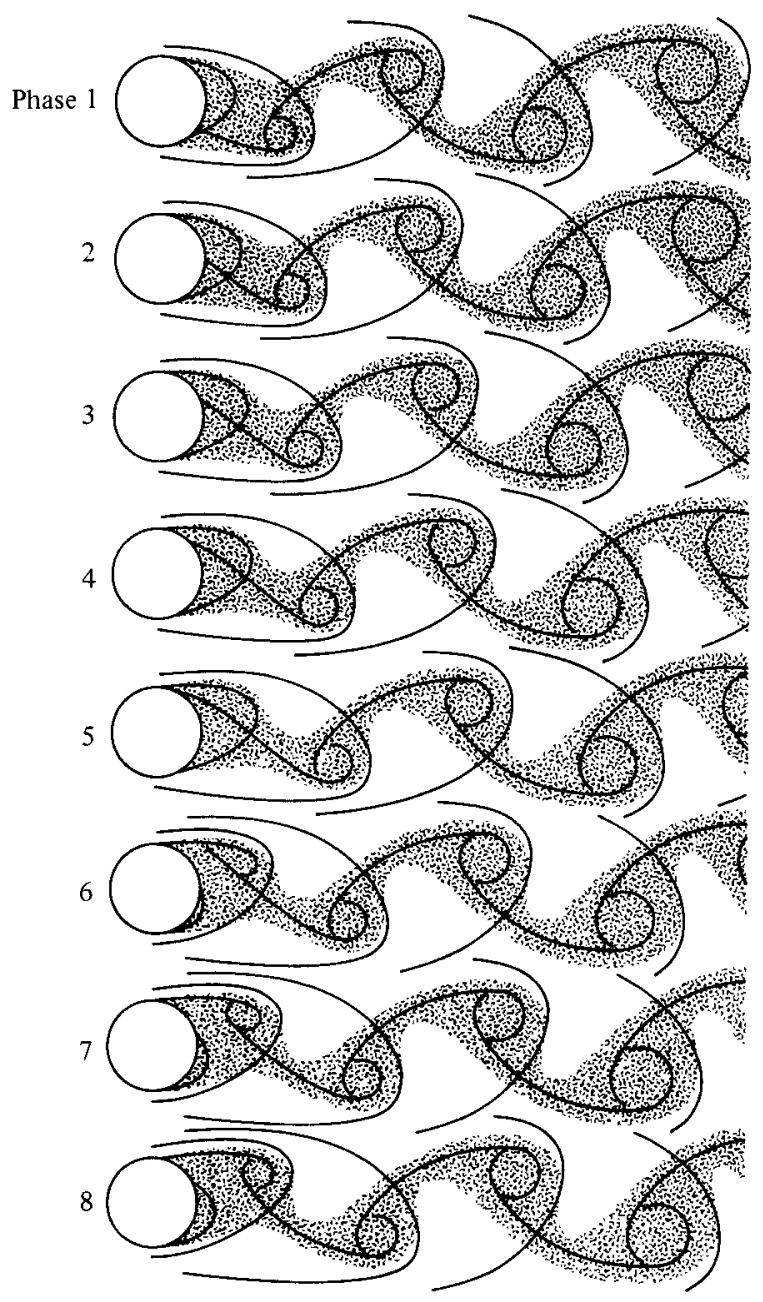

Figure 30. Conjecture for topology of material lines in turbulent vortex formation and shedding from a circular cylinder. Phase is assigned to correspond approximately to phase of experimental data in this paper.

We believe that figure 30 correctly describes the topology of material lines in the near wake, although liberties have been taken with respect to growth rate, placement of the separation points on the body, and other matters. Except for effects of dispersion, the qualitative sense of figure 30 is the same for laminar and turbulent flow; the future of each fluid element in the flow pattern is determined (say) by the time and place where it encounters the plane $x=0$. If a fluid element is not allowed to cross either of the adjacent separatrices, it must inevitably be incorporated into a specific vortex at some later time. On the other hand, fluid elements have obviously been allowed to cross separatrices in the base region, where the area of the separation bubble attached to the cylinder is shown increasing with time (note also that it is oscillation of the separatrices which ensures that a single smoke-labelled streakline can delineate the entire flow pattern, as in Zdravkovich's photographs). Consequently, figure 30 should not be viewed as a respresentation of particle paths in the real non-steady flow at any instant. The figure is an abstraction, a vision, a cartoon. In 
plane flow, singularities defined in terms of mean particle paths are of two kinds, centres and saddles. Both are moving stagnation points which have definite positions in the flow pattern at each instant. The change of position with time defines the celerity. Given the demonstration in figures 12-19 that the topology of the nonsteady mean flow cannot be perceived correctly except by an observer moving at this celerity, it is a fundamental difficulty in the case of the near wake that celerity is a vector and that it depends on position. $\dagger$ At least within the present state of the art in Lagrangian descriptions of non-steady flows, there is no way to take into account simultaneously the acceleration of the singularities away from the base region, the small component of celerity normal to the main flow, and the distortion of the flow pattern due to entrainment and growth.

Nevertheless, we believe, and we have tried to suggest in figure 30 , that the dominant topological feature of the near wake is the formation and evolution of saddle points in the flow pattern and that the saddles are crucial to any discussion of turbulence production and entrainment.

Other variables than instantaneous mean streamlines can also be useful for determining topology. For example, centres, being concentrations of mean vorticity, can be expected to remain closely associated with the same elements of fluid. Given appropriate measurements at constant phase, the centres in figure $20(a)$ might also be detected in terms of

(a) a peak in the mean vorticity (see figure $21 b$ );

(b) a peak in the intermittency (see figure $23 b$ );

(c) a peak in energy for the random turbulence (see figures $24 b$ and $25 b$ );

$(d)$ a saddle in either the periodic or random Reynolds shearing stress (see figures $26 a$ and $b$ ).

Saddles, on the other hand, need not remain closely associated with the same elements of fluid. They can be detected in terms of

(e) a zero in the mean vorticity with appreciable intermittency (see figure $21 b$ );

$(f)$ a saddle (probably traceable to dispersion) in the intermittency (see figure $23 b$ );

$(g)$ a peak in the random turbulence production (see figure $31 b$ ).

Note the important role played in five of these seven guidelines by the intermittency, which is often not measured, or by spatial derivatives of the mean velocity, which are required in order to infer vorticity or rate of turbulence production. Probably the least useful guideline is $(d)$ or $(f)$, and the most useful is $(a),(c)$ or $(g)$, depending on circumstances (cf. Hussain 1980, 1981). We will argue below that the association between saddle points and turbulence production is so strong that it seems to apply even for the global mean flow.

A very rough estimate of entrainment velocity can be made on the basis of table 3 . Near $x / d=3$ or 4 , the area of a vortex is about $4 d^{2}$. As the vortex moves one cylinder diameter downstream, this area increases by about $14 \%$. If the vortex

$\dagger$ In the absence of a global view, it may still be possible to take a local view. If the position of a given centre or saddle is known or even guessed as a function of time, its celerity can be calculated. The position at each instant can then be verified by an observer moving with this celerity. We think (although we have not proved) that an iteration scheme for closing this loop is feasible and that it would be convergent. For example, an observer moving at the velocity of the freestream in our flow would certainly be able to measure at least the streamwise separation distance $\lambda$ between vortices of like kind. This separation distance and the shedding frequency $f$ then define a phase velocity $c=\lambda f$ as a better choice of velocity for the observer. As a further example, note that the streamline pattern in figure $20(a)$ is at least qualitatively compatible with the vorticity distribution in figure $21(b)$ at the same scale and the same phase, even in the base region where the observer in figure $20(a)$ is moving at quite the wrong velocity. 
cross-section were circular and the increase in area were due to a uniformly distributed inflow, the required inflow velocity would be about $6 \%$ of $u_{\infty}$. The peripheral velocity of the same vortex can be estimated from figures $24(a)$ and $25(a)$ to be of the order of $25-50 \%$ of $u_{\infty}$. However, according to the evidence in figure $20(a)$, the entrainment velocity is demonstrably not uniform over the boundary of a vortex. Near $x / d=3.75$, for example, the velocity of the observer is nearly the same as the celerity of the flow pattern, and the pattern is nearly steady. On the downstream side of the vortex centred at about $x / d=3.13$, the mean-velocity vectors are nearly parallel to the mean interface defined by the condition $\langle\gamma\rangle=0.5$. It is a reasonable inference that entrainment along this trailing interface is small. In fact, this inference is probably valid along the lower periphery of the vortex almost as far upstream as the saddle at $(x / d, y / d)=(1.7,-0.7)+$ What is left, then, is massive entrainment at upstreamfacing interfaces.

Between any two adjacent vortices, freestream fluid is induced to flow toward and even across the wake centreline. During this process the fluid eventually crosses the intermittency boundary; i.e. it is entrained. To an observer moving downstream with the vortices, entrained fluid from both sides of the wake appears to be deflected upstream or downstream along the diverging separatrix of the saddle. Each vortex entrains fluid from both sides of the wake, but the entrainment rates are not the same. When the intermittency is superimposed on the velocity field seen by an observer moving with the vortices, as in figure $20(a)$ it is clear that the counterclockwise vortex at $x / d=3.13$ is entraining substantially more fluid from above than from below. At the Reynolds number of this experiment, once a given vortex has been formed, it entrains fluid at the highest rate from the side of the wake opposite to that from which it was shed. This is in contrast with the situation within the vortex-formation region where the net rotation sense of a given vortex reflects higher entrainment of vorticity-bearing fluid from the side of the cylinder from which it was shed. We believe that this conclusion is sound in spite of the fact that it is based on instantaneous mean streamlines rather than on time-integrated particle paths.

This conclusion has important implications in any use of a vortex street as a mixing device, including use of a thick dividing plate with a blunt trailing edge or a thin dividing plate terminated by a bluff cylinder. If the wake were used to mix together two initially unmixed reactants supplied, say, in a stoicheiometric ratio in the two streams on opposite sides of the wake, then the overall mixture ratios in the vortices, where molecular mixing must finally take place, may be far from stoicheiometric. Moreover, the effective separation of the two fluids into adjacent vortices introduces the vortex coalescence time as an important timescale for mixing, a time which may greatly exceed any of the times estimated from dimensional or gradient diffusion arguments.

\section{Turbulence production}

It was pointed out in $\$ 4$ that there is strong coupling in the present flow between the turbulence and the strain field associated with the large eddies. Further evidence for this coupling is presented in figure $31(b)$, which depicts contours of the two-dimensional turbulent energy production $\langle P\rangle$ at constant phase. The definition of production can

$\dagger$ For a graphic illustration of the fact that incorrect conclusions might be reached about entrainment when instantaneous mean streamlines are interpreted as mean particle paths in unsteady flow, compare figures 17 and 21 in Cantwell, Coles \& Dimotakis (1978). 


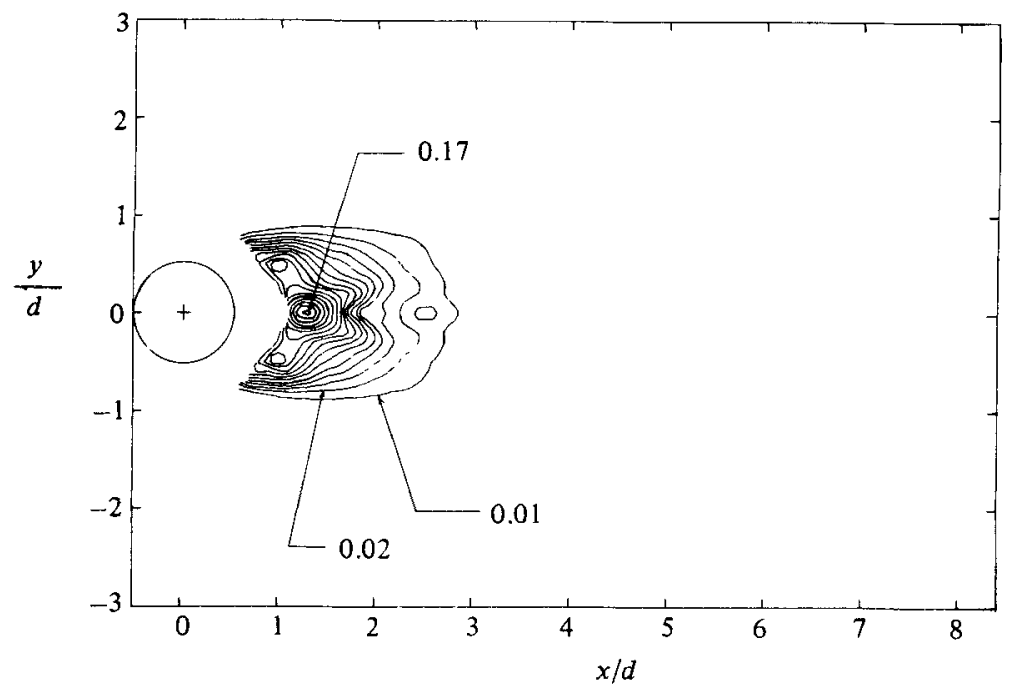

(a)

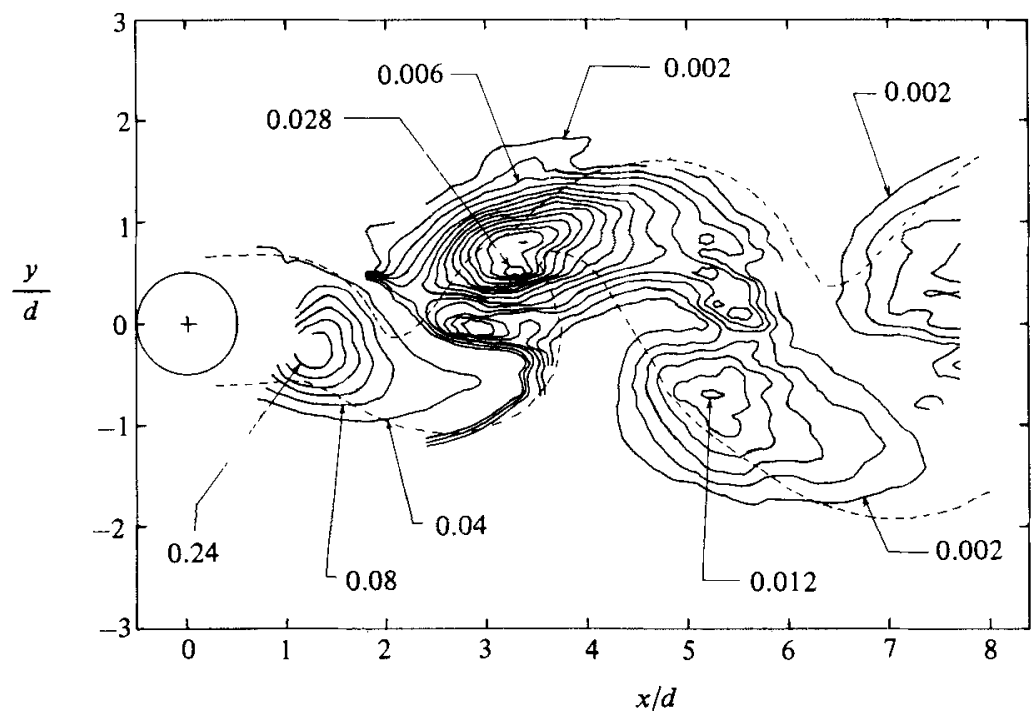

(b)

Figure 31. Contours for mean turbulent-energy production. (a) $\bar{P}$, global mean (contour interval $0.010) ;(b)\langle P\rangle$, mean at constant phase $(7,15)$. Note that the range of contour values is greater than $100: 1$. Contour interval is 0.040 for $x / d ₹ 2$; contour interval is 0.002 for $x / d>2$. Dashed line is contour $\langle\gamma\rangle=0.5$.

be obtained by continuing the derivation leading to (5):

$$
-\langle P\rangle=\left\langle u^{\prime} u^{\prime}\right\rangle \frac{\partial\langle u\rangle}{\partial x}+\left\langle u^{\prime} v^{\prime}\right\rangle\left(\frac{\partial\langle v\rangle}{\partial x}+\frac{\partial\langle u\rangle}{\partial y}\right)+\left\langle v^{\prime} v^{\prime}\right\rangle \frac{\partial\langle v\rangle}{\partial y} .
$$

The quantity $\langle P\rangle$ plays the role of an energy sink for the mean flow and an energy source for what we call random turbulent fluctuations. No significant regions of negative production were found in the present data at any phase of the motion. 
Comparison of figures $20(a), 26(b)$, and $31(b)$ shows that the peaks in production lie close to the peaks in $\left\langle u^{\prime} v^{\prime}\right\rangle$, and also close to the saddles in the flow pattern. Some experimental data in table 4 include values of the various quantities in (13) for the two saddles lying at $(x / d=3.1, y / d=1.0)$ and at $(x / d=5.5, y / d=-1.0)$ at phase $(7,15)$. According to the table, it is the middle term of $(13)$ that dominates the production.

Similar conclusions about turbulence production have been reached by Hussain (1980, 1981 ; see also Hussain \& Zaman 1981), who studied the flow at constant phase in an axisymmetric shear layer. Hussain noted that in this flow turbulence was produced mainly in the braids between the large vortices and then transported to and accumulated in the vortices. However, Hussain evaluated only the middle term in (13), and he did not comment at any length either on topology or on the mechanisms of turbulence production. We believe that these two concepts need comment.

Equation (13) defines the turbulence production as the scalar product $\tau_{i j} \partial u_{i} / \partial x_{j}$ (summed over $i=1,2$ and $j=1,2$ for the case of two-dimensional mean flow). The numerical value of $\langle P\rangle$ is not affected by a Galilean transformation or by a change in the direction of the coordinate axes. However, rotation of the coordinate system can be a very useful device for weighing the work done by shearing stresses $(i \neq j)$ against the work done by normal stresses $(i=j)$.

For this purpose, let the original contraction over both indices be represented in a highly schematic form as

$$
P=\left(\begin{array}{ll}
\boldsymbol{T} & \boldsymbol{T} \\
\boldsymbol{T} & \boldsymbol{T}
\end{array}\right):\left(\begin{array}{ll}
\boldsymbol{G} & \boldsymbol{G} \\
\boldsymbol{G} & \boldsymbol{G}
\end{array}\right)
$$

where $\boldsymbol{T}$ stands for $\tau_{i j}$ and $\boldsymbol{G}$ for $\partial u_{i} / \partial x_{j}$. By a suitable change of axes, the turbulence production can be expressed in such a way that only shearing stresses enter into the calculation of $P$. There are two possibilities; either

or

$$
\begin{aligned}
& P=\left(\begin{array}{ll}
\mathbf{0} & \boldsymbol{T} \\
\boldsymbol{T} & \mathbf{0}
\end{array}\right):\left(\begin{array}{ll}
\boldsymbol{G} & \boldsymbol{G} \\
\boldsymbol{G} & \boldsymbol{G}
\end{array}\right) \quad \text { (laminar only) } \\
& P=\left(\begin{array}{ll}
\boldsymbol{T} & \boldsymbol{T} \\
\boldsymbol{T} & \boldsymbol{T}
\end{array}\right):\left(\begin{array}{ll}
\boldsymbol{0} & \boldsymbol{G} \\
\boldsymbol{G} & \mathbf{0}
\end{array}\right) \quad \text { (incompressible) }
\end{aligned}
$$

Similarly, there are two possibilities for expressing the turbulence production in such a way that only normal stresses enter into the calculation. Either

or

$$
\begin{aligned}
& P=\left(\begin{array}{ll}
\boldsymbol{T} & \mathbf{0} \\
\mathbf{0} & \boldsymbol{T}
\end{array}\right):\left(\begin{array}{ll}
\boldsymbol{G} & \boldsymbol{G} \\
\boldsymbol{G} & \boldsymbol{G}
\end{array}\right) \quad\left(\tau_{i j}\right. \text { symmetric) } \\
& P=\left(\begin{array}{ll}
\boldsymbol{T} & \boldsymbol{T} \\
\boldsymbol{T} & \boldsymbol{T}
\end{array}\right):\left(\begin{array}{cc}
\boldsymbol{G} & \mathbf{0} \\
\boldsymbol{O} & \boldsymbol{G}
\end{array}\right) \quad \text { (irrotational) }
\end{aligned}
$$

The first form (14) is not relevant for turbulent flow, because the trace of the Reynolds-stress tensor (the sum of the Reynolds normal stresses) is both different from zero and invariant to rotation.

The second form (15) can always be achieved if the fluid is incompressible, because the trace of the velocity-gradient tensor $\partial u_{i} / \partial x_{j}$ vanishes by continuity. The required change of axes is a counterclockwise rotation through the angle $\theta$ defined by

$$
\tan 2 \theta=-\frac{\frac{\partial u}{\partial x}-\frac{\partial v}{\partial y}}{\frac{\partial u}{\partial y}+\frac{\partial v}{\partial x}}
$$




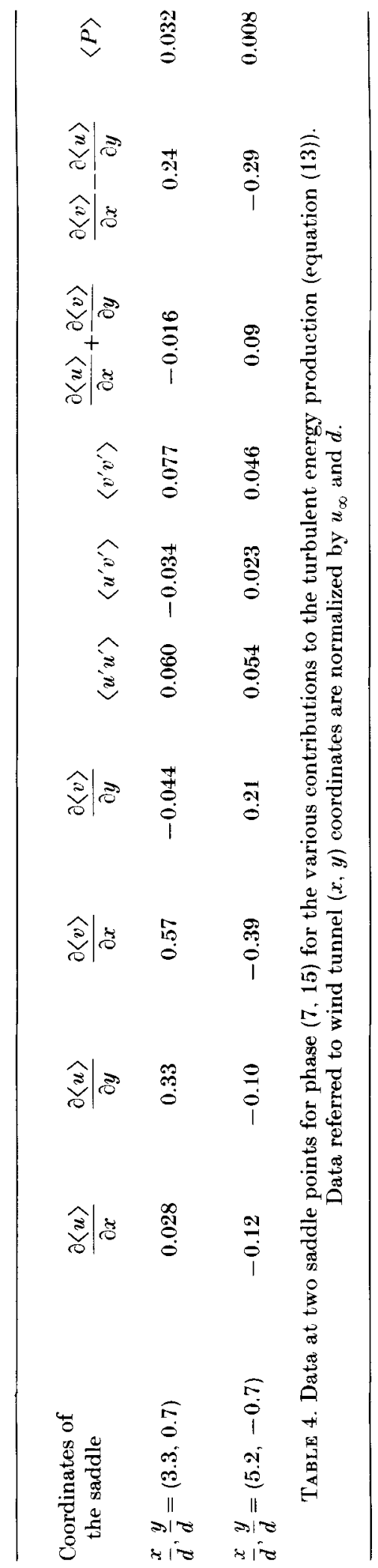


The third form (16) can also always be achieved by transferring to principal axes associated with the symmetric Reynolds stress tensor $\tau_{i j}$. The required counterclockwise rotation angle is given by

$$
\tan 2 \theta=\frac{2 \tau_{x y}}{\tau_{x x}-\tau_{y y}} .
$$

Finally, the fourth form (17) can always be achieved since $\tau_{i j}$ is symmetric and $\tau_{i j} \partial u_{i} / \partial x_{i}=\frac{1}{2} \tau_{i j}\left(\partial u_{i} / \partial x_{i}+\partial u_{i} / \partial x_{i}\right)$. Hence the desired axes are the principal axes of the rate-of-strain tensor. From the point of view of (17) an important exceptional case is irrotational flow, for which the velocity-gradient tensor becomes symmetric. In this case, the required counterclockwise rotation angle is given by

$$
\tan 2 \theta=\frac{\frac{\partial u}{\partial y}+\frac{\partial v}{\partial x}}{\frac{\partial u}{\partial x}-\frac{\partial v}{\partial y}}
$$

The coordinate axes defined by $(20)$ are evidently at $45^{\circ}$ to the coordinate axes defined by (18).

After these preliminaries, we are better prepared to discuss the concept of turbulence production. For classical channel flow, for example, and less rigorously for any two-dimensional steady mean flow described by a boundary-layer approximation, the production already has the form

$$
P=\left(\begin{array}{cc}
\boldsymbol{T} & \boldsymbol{T} \\
\boldsymbol{T} & \boldsymbol{T}
\end{array}\right):\left(\begin{array}{ll}
\mathbf{0} & \boldsymbol{G} \\
\mathbf{0} & \mathbf{0}
\end{array}\right)
$$

in the original coordinates. Only $\partial u / \partial y$ is non-zero, with $\theta=0$ from (18). A natural inference, at least within the limitations of global averaging, is that turbulence production in such flows is by shearing strain, through a mechanism which can perhaps be visualized as an instability of Kelvin-Helmholtz type. But these same flows can also be treated using the form (16), which involves only turbulent normal stresses and thus seems to imply production by the different mechanism of vortex stretching. When the example of a channel flow is worked out in detail, the apparent contradiction turns out to be one of form and not of substance. No purpose is served by considering (16). In short, this example suggests that coordinate transformations which operate on the stress tensor $\tau_{i j}$ can be misleading, and in any event do not lead to useful conclusions about turbulence production in flows which are not of boundarylayer type. It is rather the velocity-gradient tensor $\partial u_{i} / \partial x_{j}$ which has to be manipulated so as to represent correctly the rate-of-strain field of the mean flow, and particularly of the mean flow near coherent structures observed at constant phase.

It remains to consider (17) and the special case of a mean flow that is both turbulent and locally irrotational. Such flows are so rare that they deserve special attention when they do occur; and they occur at saddles in the flow patterns encountered in the present work and in the work by Hussain cited earlier. The fact that calculation of $\langle P\rangle$ in tunnel coordinates is dominated by the middle term in (13) is apparently an artifact of the choice of coordinates. Consider conditions at the first saddle in table 4 . In the original coordinates, with the dominant terms underlined,

$$
-\langle P\rangle=\left(\begin{array}{lr}
0.060 & -0.034 \\
-0.034 & 0.077
\end{array}\right):\left(\begin{array}{cc}
0.028 & \underline{0.330} \\
\underline{0.570} & -0.044
\end{array}\right)=-0.032 .
$$


The rotation required by $(20)$ is defined by

$$
\tan 2 \theta=\frac{0.330+0.570}{0.028+0.044}=12.5
$$

from which $\theta=42.7^{\circ}$, in reasonable agreement with the direction of the separatrices in figure $20(a)$. In the rotated coordinates

$$
-\langle P\rangle=\left(\begin{array}{ll}
0.034 & 0.006 \\
0.006 & 0.103
\end{array}\right):\left(\begin{array}{ll}
\underline{0.444} & -0.120 \\
0.120 & -\underline{0.460}
\end{array}\right)=-0.032
$$

This example is less than perfect, because continuity is not quite satisfied experimentally (div $\boldsymbol{u}$ is -0.016 ), and the local flow is not quite irrotational (curl $\boldsymbol{u}$ is 0.24 , compared with a value of 1.99 at the adjacent vorticity peak). Nevertheless, the data support our claim that it is not the peak in $\left\langle u^{\prime} v^{\prime}\right\rangle$ which accounts for the large turbulence production, but rather the strong strain field near the saddle. A plausible inference is that the mechanism for the observed turbulence production is essentially pure extension or vortex stretching. As a final contribution to this topic, the globally averaged turbulence production is shown in figure $31(a)$. The distribution of $\bar{P}$ is essentially bimodal about the plane of symmetry of the wake except for a region on the centreline at about $x / d=1.3$, where there is a sharp peak in $\bar{P}$. The location of this peak is quite close to the wake closure point of the mean velocity field (ef. figure 10). Note that this closure point is a saddle of the mean streamline pattern. Moreover, the vanishing of the global shearing stress and of the derivatives $\partial \bar{u} / \partial y$ and $\partial \bar{v} / \partial x$ on the plane of symmetry leaves only vortex stretching as a mechanism for turbulence production near the saddle even in the globally averaged flow.

\section{Concluding remarks}

The present experiments do not shed much new light on the mechanics of vortex formation very close to the cylinder, say for $0.5<x / d<1.5$. What is certain is that random fluctuations in the base region are very large, with both $u^{\prime} / u_{\infty}$ having r.m.s. values of 0.4 or more, according to figures $24(b)$ and $25(b)$. Turbulent mixing by convection at inviscid scales, rather than turbulent diffusion at viscous scales, is probably a sufficient mechanism to account for much of the cancellation of mean vorticity in the base region. A decision on this point would be greatly aided by simultaneous direct measurements of fluctuations in vorticity and velocity.

It may or may not be useful to try to define shedding of a vortex from the base region of a bluff body as a distinct event. Gerrard (1966) suggests using as a criterion the first penetration of non-turbulent freestream fluid across the wake centreline. If the interface is taken as the contour $\langle\gamma\rangle=0.5$, this event occurs in the present data at about phase $(7,15$ ) at about $x / d=2.1$ (cf. figure $23 b$ ). The region of strongest penetration lies farther downstream, at about $x / d=3$, where in figure $23(a)$ there is a local minimum in $\bar{\gamma}$ on the wake centreline at which $\bar{\gamma}=0.6$. We prefer another criterion (mentioned in passing by Gerrard) which is that the mean vorticity has become negligible at the saddle next upstream of the vortex being shed. This event again occurs at about phase $(7,15)$ at roughly $(x / d, y / d)=(1.6,-0.8)$ (cf. figure $21 b$ ). Each vortex, once shed, moves downstream under the influence of the induced velocity field of the other vortices of the system, with further changes of mean vorticity occurring relatively slowly by transfer across adjacent saddles.

Our data confirm the finding by other investigators that less than half of the circulation generated at one side of the eylinder during one shedding cycle appears 
in the vortices downstream. We consider the complementary statement to be equally remarkable; nearly half of the shed circulation does appear in concentrated form. There is a powerful tendency for vorticity to accumulate in coherent structures. The mechanisms which make these structures relatively stable and permanent are not yet clear, but the present research does identify some important elements.

A plausible conjecture is that turbulence production near saddles is carried out primarily by stretching of small-scale vorticity oriented along the diverging separatrices, which cross at right angles if there is no local mean vorticity (this remark does not take into account the small transport of mean vorticity needed to account for the decay of circulation, as discussed in $\$ 4$ under 'vortex motion'. Given that turbulence is already present near the saddles, this turbulence is continuously amplified to maintain a state of equilibrium. If the entrainment velocity, which must depend on the properties of the local turbulence, were not maintained at a sufficiently high value, the flow would break up into islands of turbulence near each centre, and this turbulence would presumably decay. Thus the turbulence is strongly coupled to the strain field associated with the non-steady mean velocity field. In fact, the mechanism has something in common with the one described for the plane laminar mixing layer by Corcos \& Sherman (1976). The vortices, once formed and shed, generate a strain field which guarantees that there is an energetic level of turbulence production near the saddles. The newly turbulent fluid, while it is being transported to the centres, controls the local propagation velocity of the interfaces in such a way as to preserve the overall geometry of the growing turbulent region and thus of the coherent structures. It is possible, and even likely, that the phenomenon of turbulence production by vortex stretching near saddles is a general, and even universal, property of free turbulent shear flow's, even when no coherent structure is detectable by any means currently available. The role of three-dimensionality at large scales therefore urgently needs attention if a connection is ever to be made with similarity arguments which require the Reynolds number for a round wake to decrease with increasing distance (so that the round wake must eventually decay) but require the global Reynolds number for a plane wake to be constant.

It is important in figure $20(a)$ that the entrainment process is not symmetric with respect to the diverging separatrix of the saddles. In fact, the saddles appear to lie on the boundary $\langle\gamma\rangle=0.5$, where part of the fluid tends to be deflected without being strongly entrained (this property probably does not hold for the mixing layer, for which the vorticity in the coherent structures always has the same sense). Further evidence for unsymmetric activity at saddles between vortices can be found in the photographs in figure 32. Figure $32(a)$ is a schlieren photograph taken by Thomann (1959) of the near wake of a wedge at $M_{\infty}=0.5$. The arrow points to a linear feature which connects two adjacent vortices and lies in the direction of the diverging separatrix. This feature is visible because of strong density gradients at the saddle. Figure $32(b)$ is a photograph taken by Meijer (1965) of the near wake of a cavitating flapped hydrofoil. Here the arrow points to a linear bubble which indicates a region of low pressure near the saddle, rather than the high pressure normally associated with a stagnation point. We may speculate that in both cases the features are related to intense organized concentrations of streamwise vorticity aligned with the diverging separatrix of the saddle. Vortex stretching at saddles must play a key role in allowing entrainment rates to be amplified far above what would be expected on the basis of local turbulence intensity alone. In short, what we have called random turbulence in this paper is neither weak, nor homogeneous, nor isotropic; it has structure.

Although we have been obliged to adopt the concept of Reynolds averaging for 


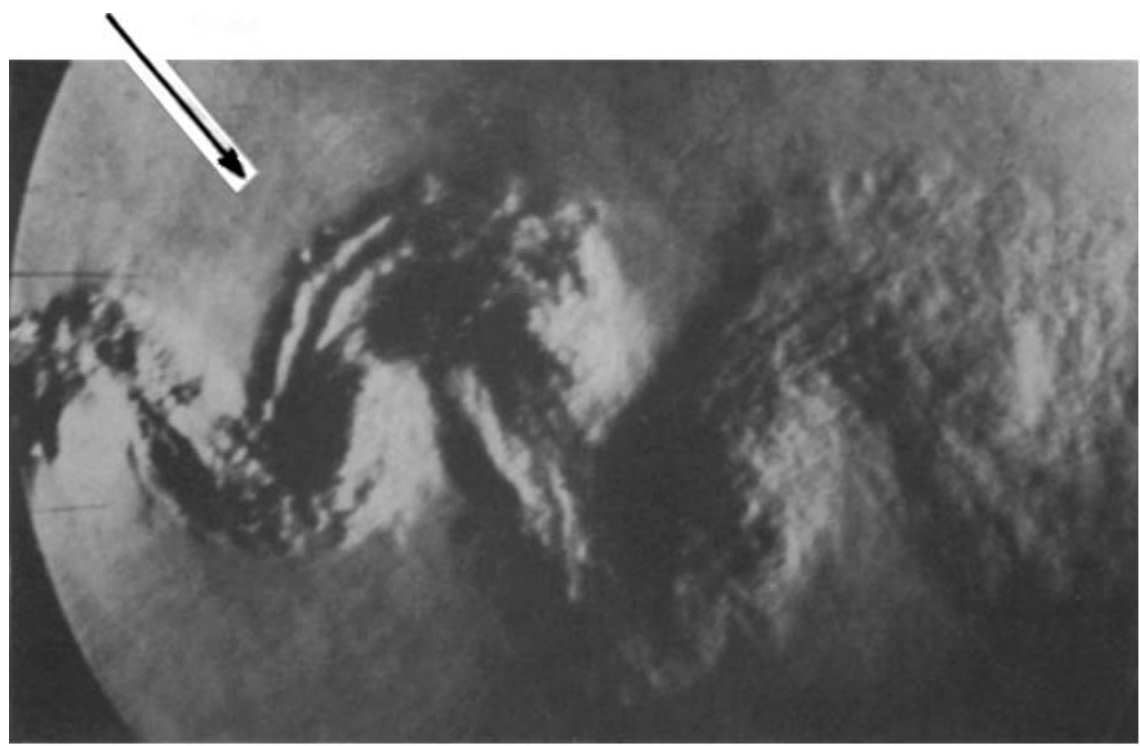

(a)

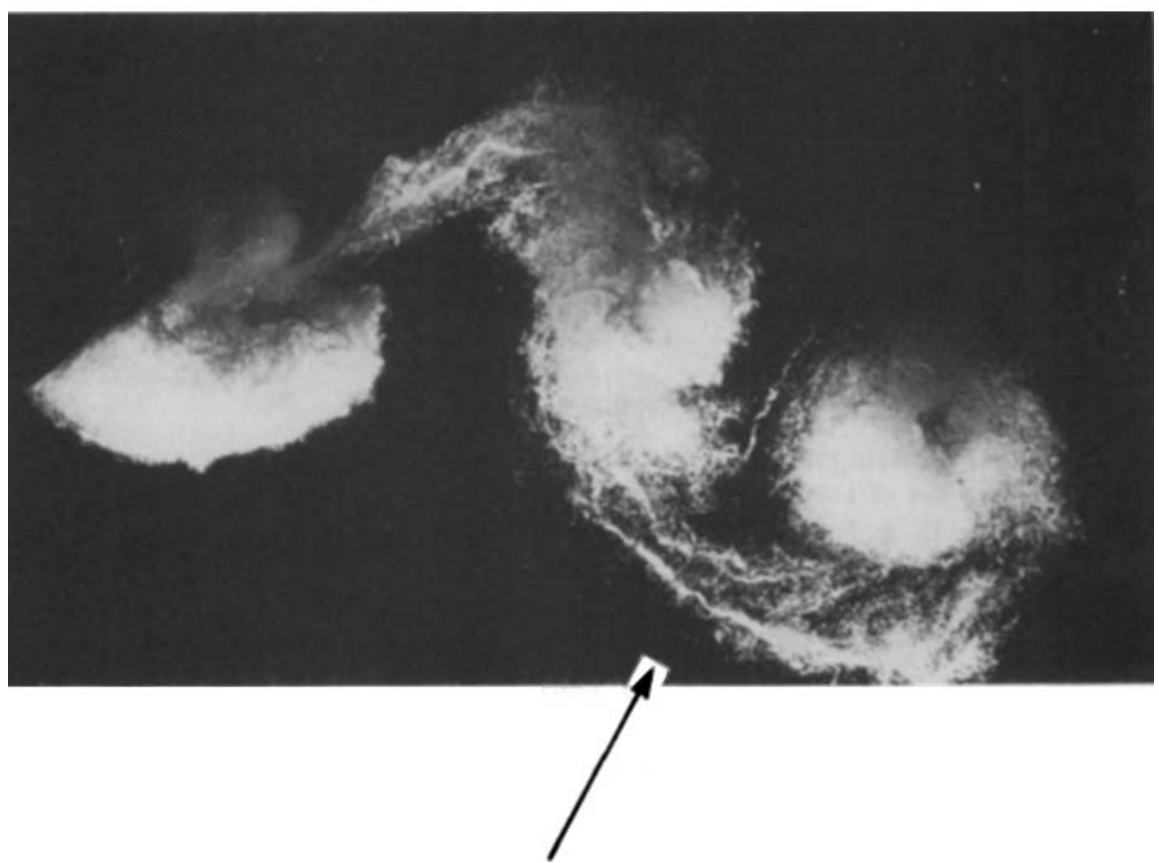

(b)

Figure 32. Visual evidence for organized structure in the saddle region between vortices: $(a)$ near wake of a wedge at $M_{\infty}=0.5$ (from Thomann 1959); $(b)$ near wake of a cavitating flapped hydrofoil (from Meijer 1965).

the flow at constant phase in order to have access to language suitable for deseribing production of turbulent energy and other phenomena, we have several reservations. The conventions of turbulence imply that turbulent energy is produced at the largest scale of the flow (here the scale of the individual vortices) and is then transferred by a cascade process to smaller scales. The evidence of the present experiment, however, 
is that a substantial fraction of the turbulent energy is produced primarily at intermediate or even small scales near saddles in the flow pattern and is later transported to and accumulated at centres whose larger scale may be deceptively conspicuous in measured spectra or correlations. The conventions of turbulence also imply, at least in the context of global Reynolds averaging and a boundary-layer approximation, that energy is produced in the streamwise or $u$-component and is later transferred to the $v$ - and $w$-components by whatever mechanism is represented by the pressure-strain covariance in the Reynolds-averaged turbulent-energy equation. This conclusion certainly cannot hold for fully developed coherent structures if the dominant turbulence production mechanism is vortex stretching at saddles, and any boundarylayer approximation for flow at constant phase should therefore be avoided.

Finally, the relevance of the present data to conventional turbulence modelling is epitomized by (6) and by figures 24-29. This material is based on the triple decomposition of (1), in which the departure of any variable from its global mean value is represented by the sum of a periodic component and a random component. For the mean flow at constant phase, the two components have generally comparable magnitudes but very different topologies, and the difference extends to the global mean in the case of the streamwise velocity component. The importance of both periodic and random fluctuations means that neither an inviscid rotational model nor a gradient-diffusion model alone can account properly for entrainment and mixing. In particular, the ruinous loss of detail which is encountered when data at constant phase are averaged over time goes some way toward explaining why global turbulence modelling is more art than science.

The research reported here was supported by NASA Grant NGL 05-002-229, with supplementary support during the later stages of data processing from NSF Grant ENG 77-23541 and NASA Grant NCC 2-21.

\section{REFERENCES}

Cantwell, B. J. 1976 A flying hot wire study of the turbulent near wake of a circular cylinder at a Reynolds number of 140,000. Ph.D. thesis, Calif. Inst. Tech.

Cantwell, B., Coles, D. \& Dimotakis, P. 1978 Structure and entrainment in the plane of symmetry of a turbulent spot. J. Fluid Mech. 87, 641-672.

Colfs, D. 1981 Prospects for useful research on coherent structure in turbulent shear flow. Proc. Indian Acad. Sci. (Engng Sci.) 4, 111-127.

Coles, D., Cantwell, B. \& Wadcock, A. 1978 The flying hot wire and related instrumentation. $N A S A$ CR 3066.

Coles, D. \& WADCock, A. 1979 Flying-hot-wire study of flow past a NACA 4412 airfoil at maximum lift. $A I A A J$. 17, 321-329 (AIAA Paper 78-1196).

Corcos, G. M. \& Sherman, F. S. 1976 Vorticity concentration and the dynamics of unstable free shear layers. J. Fluid Mech. 73, 241-264.

DAviEs, M. E. 1976 A comparison of the wake structure of a stationary and oscillating bluff body, using a conditional averaging technique. J. Fluid Mech. 75, 209-231.

Drescher, H. 1956 Messung der auf querangeströmte Zylinder ausgeübten zeitlich veränderten Drücke. Z. Flugwiss. 4, 17-21.

Dyment, A., Gryson, P. \& Ducruet, C. 1980 Unpublished paper at Euromech Colloq. no. 135; some photographs are included in M. Van Dyke An Album of Fluid Motion, pp. 130-131 (Parabolic, 1982). 
Gerrard, J. H. 1966 The mechanies of the formation region of vortices behind bluff bodies. $J$. Fluid Mech. 25, 401-413. .

GoRECKI, J. P. 1960 An investigation of temperature fluctuations on bluff bodies. Ph.D. thesis, Calif. Inst. Tech.

Gowen, F. E. \& Perkins, E. W. 1953 Drag of circular cylinders for a wide range of Reynolds numbers and Mach numbers. NACA TN 2960.

Hooker, S. G. 1936 On the action of viscosity in increasing the spacing ratio of a vortex street. Proc. R. Soc. Lond. A 154, 67-89.

Hussain, A. K. M. F. 1980 Coherent structures and studies of perturbed and unperturbed jets. In The Role of Coherent Structures in Modelling Turbulence and Mixing (ed. J. Jimenez). Lecture Notes in Physics, vol. 136, pp. 252-291. Springer.

Hussain, A. K. M. F. 1981 Role of coherent structures in turbulent shear flows. Proc. Indian Acad. Sci. (Engng Sci.) 4, 129-175.

Hussain, A. K. M. F. \& Zaman, K. B. M. Q. 1981 The 'preferred mode' of the axisymmetric jet. J. Fluid Mech. 110, 39-71.

KáRmán, T. 1912 Über den Mechanismus des Widerstandes, den ein bewegter Körper in einer Flüssigkeit erfährt. Göttingen Nachr., Math.-Phys. Klasse, pp. 547-556.

Kline, S. J., Cantwell, B. J. \& Lilley, G. M. 1981 1980-81 AFOSR-HTTM-Stanford Conf. on Complex Turbulent Flows, vol. I. Thermosciences Div., Mech. Engng Dept, Stanford Univ.

Kolmogorov, A. 1941 The local structure of turbulence in incompressible fluid for very large Reynolds numbers. C.R. (Dokl.) Acad. Sci. URSS 30, 301-305.

Matsur, T. 1981 Flow visualization studies of vortices. Proc. Indian Acad. Sci. (Engng Sci.) 4, $239-257$.

MeiJer, M. C. 1965 Pressure measurement on flapped hydrofoils in cavity flows and wake flows. Hydrodyn. Lab., Calif. Inst. Tech., Rep. E-133.2 (and private communication).

Naumans, A., Morsbach, M. \& Kramer, C. 1966 The conditions for separation and vortex formation past cylinders. In Separated Flows; AGARD Conf. Proc, no. 4, part 2, pp. 539-574.

Nielsen, K. W. 1970 Vortex formation in a two-dimensional periodic wake. Ph.D. thesis, Oxford Univ.

Owen, F. K. \& Johnson, D. A. 1980 Measurements of unsteady vortex flow fields. $A I A A J .18$, 1173-1179 (AIAA Paper 78-18).

Peake, D. J. \& Tobak, M. 1980 Three-dimensional interactions and vortical flows with emphasis on high speeds. AGARDograph 252.

Perry, A. E., Chong, M. S. \& Lim, T. T. 1982 The vortex-shedding process behind two-dimensional bluff bodies. J. Fluid Mech. 116, 77-90.

Perry, A. E. \& Morrison, G. L. 1971 A study of the constant-temperature hot-wire anemometer. J. Fluid Mech. 47, 557-599.

Perry, A. E. \& WATmuff, J. H. 1981 The phase-averaged large-scale structures in threedimensional turbulent wakes. J. Fluid Mech. 103, 33-51.

Reynolds, W. C. \& Hussain, A. K. M. F. 1972 The mechanies of an organized wave in turbulent shear flow. Part 3. Theoretical models and comparisons with experiments. J. Fluid Mech. 54, $263-288$.

Rosнко, A. 1954 On the drag and shedding frequency of two-dimensional bluff bodies. $N A C A$ TN 3169.

Ryan, L. F. 1951 Experiments on aerodynamic cooling. ETH Zürich, Inst. für Aerodyn., Mitt. nr. 18.

Sohmidt, D. W. \& Tilmann, P. M. 1972 Über die Zirkulations-entwicklung in Nachläufen von Rundstäben. Acustica 27, 14-22.

Stack, J. 1941 Compressibility effects in aeronautical engineering. NACA Confidential Rep. no. 246 (declassified 1967).

Thomans, H. 1959 Measurements of the recovery temperature in the wake of a cylinder and of a wedge at Mach numbers between 0.5 and 3. Aero. Res. Inst. Sweden (FFA) Rep. no. 84. 
Tutu, N. AND Chevray, R. 1975 Cross-wire anemometry in high intensity turbulence. J. Fluid Mech. 71, 785-800.

WAGNER, W.J. 1976 Experimentelle Untersuchung der Nachlaufströmungen im Nahbereich hinter Rundstäben mittels Messung der Temperatureschwankungen. Mitt. MPI unde AVA, Göttingen, nr. 62.

WLEZIEN, R. W. \& WAY, J. L. 1979 Techniques for the experimental investigation of the near wake of a circular cylinder. $A I A A J .17,563-570$ (AIAA Paper 78-1197).

ZDRavkovich, M. M. 1969 Smoke observations of the formation of a Kármán vortex street. J. Fluid Mech. 37, 491-496. 